\title{
Akute Appendizitis - \\ Verlässlichkeit der präoperativen Diagnostik, chirurgisches Management und Vergleich des intraoperativen und histopathologischen Befundes
}

\author{
INAUGURAL-DISSERTATION \\ zur Erlangung des Doktorgrades \\ für Zahnheilkunde \\ der Medizinischen Fakultät der \\ Georg-August-Universität zu Göttingen \\ vorgelegt von \\ Ulrike Kopsch \\ aus \\ Leipzig
}

Göttingen 2016 
Dekan:

I. Berichterstatter:

II. Berichterstatter/in:

III. Berichterstatter/in:
Prof. Dr. rer. nat. H. K. Kroemer

Prof. Dr. med. O. Kollmar

Prof. Dr. med. Dr. Martin Bauer

Tag der mündlichen Prüfung: $\quad$ 19.09.2016 


\section{Inhaltsverzeichnis}

$1 \quad$ Einleitung

$1.1 \quad$ Geschichtliche Entwicklung

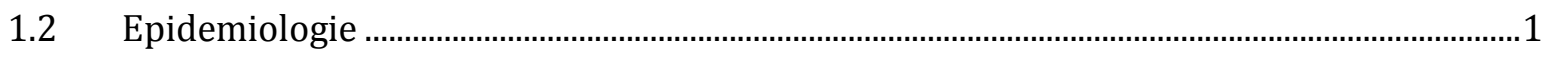

$1.3 \quad$ Anatomie und Pathogenese .................................................................................................

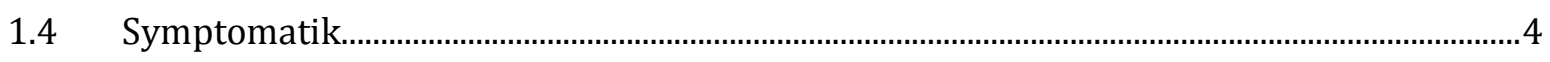

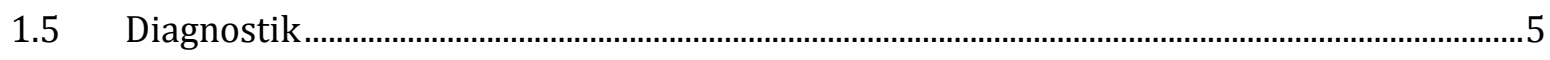

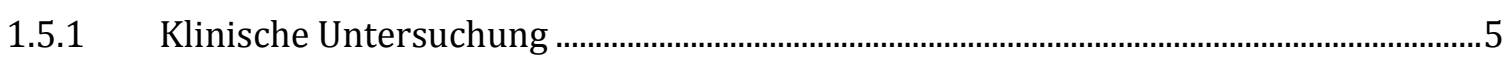

1.5.2 Laborchemische Untersuchung....................................................................................

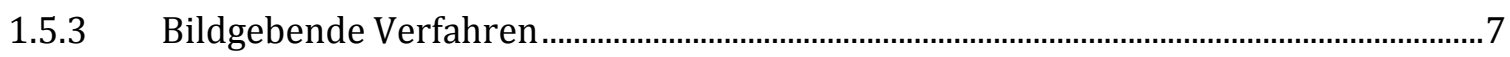

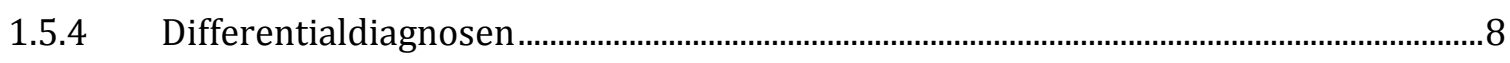

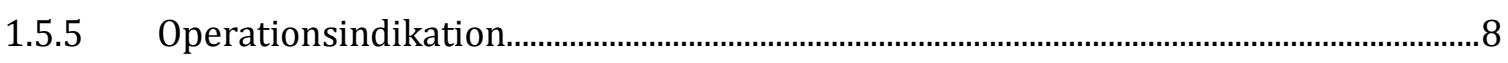

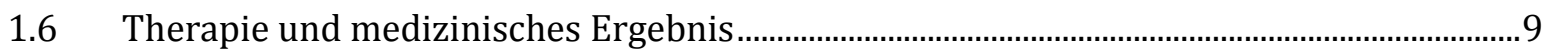

$1.7 \quad$ Fragestellung und Zielsetzung der Arbeit ................................................................................ 11

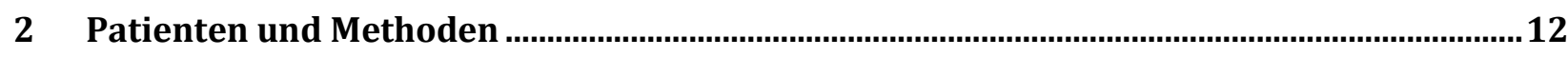

$2.1 \quad$ Datenerhebung und statistische Auswertung …………........................................................... 12

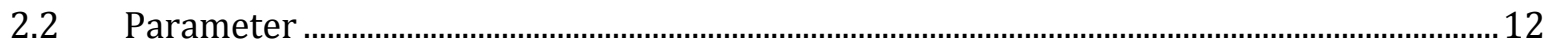

2.2.1 Demographische Daten .............................................................................................. 12

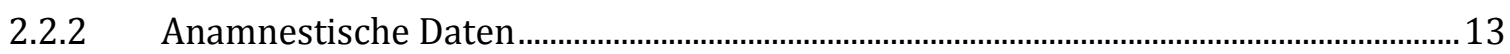

2.2.3 Untersuchungsbefund und apparative Diagnostik ………………................................ 13

2.2.4 Perioperative Daten ....................................................................................................... 14

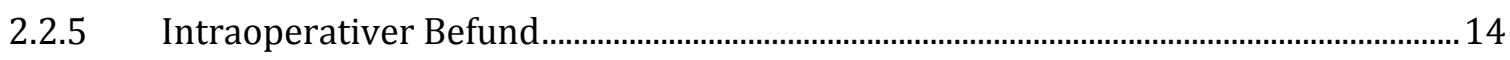

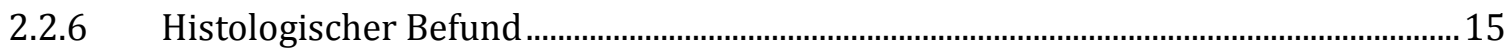

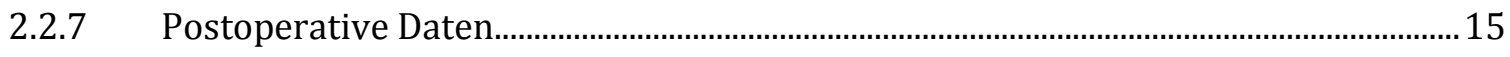

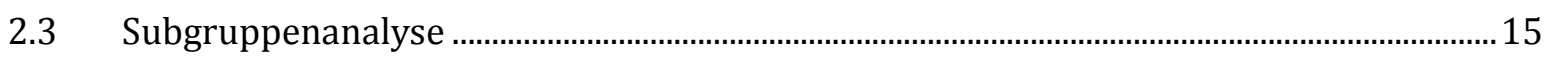

2.4 Gegenüberstellung intraoperativer und histologischer Befund ............................................16 


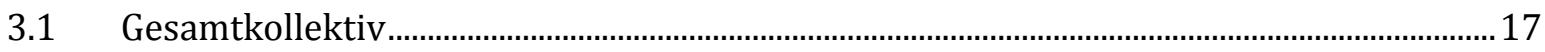

3.1.1 Demographische Daten ..................................................................................................

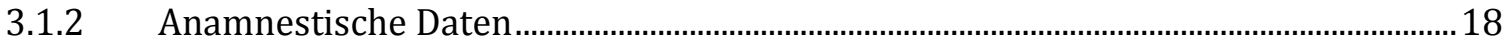

3.1.3 Untersuchungsbefund und apparative Diagnostik ...................................................... 19

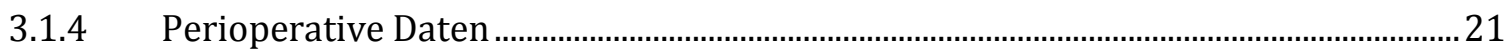

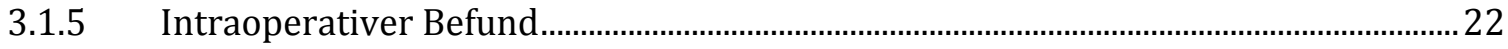

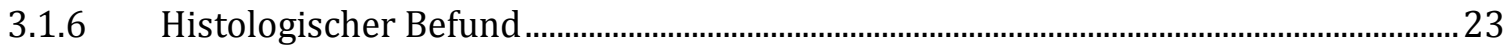

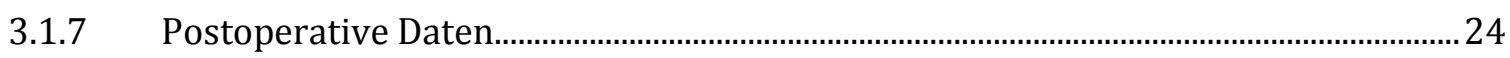

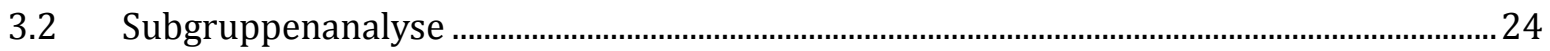

3.2.1 Intraoperativer Befund nicht-akute vs. akute Appendizitis..........................................2.

3.2.2 Histologischer Befund nicht-akute vs. akute Appendizitis ........................................... 27

3.2.3 Nicht-komplizierter vs. komplizierter postoperativer Verlauf...................................... 29

3.2.4 Nicht-perforierte vs. perforierte Appendizitis..................................................................... 32

3.2.5 Keine Peritonitis vs. Peritonitis........................................................................................... 35

3.3 Gegenüberstellung intraoperativer und histologischer Befund ........................................... 38

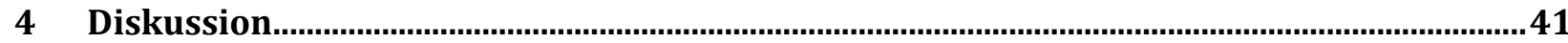

4.1 Präoperative Prädiktoren der akuten Appendizitis und des komplikationsbehafteten Krankheitsverlaufs

4.2 Einfluss der Aufnahme-Schnitt-Dauer auf den Schweregrad der Appendizitis und Einfluss eines fortgeschrittenen Befundes auf den postoperativen Verlauf...............46

4.3 Übereinstimmung zwischen chirurgischem und histologischem Befund...........................50

4.4 Einschränkungen der Methodik …………................................................................................ 53

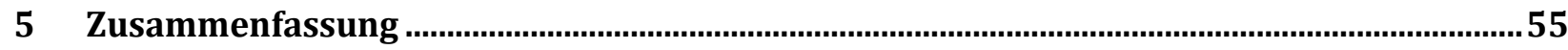

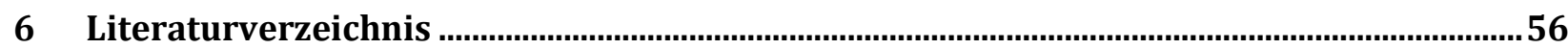




\section{Abkürzungsverzeichnis}

\begin{tabular}{|c|c|}
\hline ASD & Aufnahme-Schnitt-Dauer \\
\hline CT & Computertomographie \\
\hline${ }^{\circ} \mathrm{C}$ & Grad Celsius \\
\hline $\mathrm{y}$ & Jahr \\
\hline $\mathrm{kg}$ & Kilogramm \\
\hline LA & laparoskopische Appendektomie \\
\hline 1 & Liter \\
\hline $\mathrm{m}$ & Meter \\
\hline$\mu \mathrm{l}$ & Mikroliter \\
\hline $\mathrm{mg} / \mathrm{l}$ & Milligramm je Liter \\
\hline $\mathrm{mm}$ & Millimeter \\
\hline $\min$ & Minute \\
\hline MW & Mittelwert \\
\hline $\mathrm{OA}$ & offene Appendektomie \\
\hline o.p.B. & ohne pathologischen Befund \\
\hline $\mathrm{OP}$ & Operation \\
\hline$\%$ & Prozent \\
\hline $\mathrm{p}$ & p-Wert \\
\hline Sono & Sonographie \\
\hline SD & Standardabweichung \\
\hline $\mathrm{h}$ & Stunde \\
\hline vs. & versus \\
\hline $\mathrm{cm}$ & Zentimeter \\
\hline
\end{tabular}




\section{Einleitung}

\subsection{Geschichtliche Entwicklung}

Das Krankheitsbild der akuten Appendizitis ist eine der häufigsten Ursachen für ein akutes Abdomen. Etwa 135.000 jährlich in Deutschland durchgeführte Appendektomien verdeutlichen den Stellenwert dieser viszeralchirurgischen Therapie im Rahmen der chirurgischen Notfallversorgung (Sahm et al. 2013). Während heute jedoch eine vollständige Genesung der betroffenen Patienten erwartet wird, zeichnet die historische Betrachtung der Erkrankung ein vollkommen anderes Bild.

Obwohl eine anatomische Beschreibung der Appendix durch eine 1492 erstellte Zeichnung Leonardo da Vincis dokumentiert ist, existierte das Wissen um die pathologischen Zusammenhänge der Appendizitis damals noch lange nicht. 1711 beschrieb erstmals der Nürnberger Anatom Lorenz Heister eine schwarze, adhärente und eitergefüllte Appendix während einer Leichensektion. Der Brite Claudius Amyand führte 1735 die erste dokumentierte Appendektomie aus dem Bruchsack einer Skrotalhernie eines Kindes durch. Erst 1886 prägte der HarvardProfessor Reginald Fitz den medizinischen Terminus „Appendizitis“ (Hamill et al. 2014) und ebnete so den Weg für die erste auf deutschem Boden erfolgreich diagnostizierte und chirurgisch therapierte Appendizitis 1888 durch Max Schüler in Berlin (Sahm et al. 2011). Als standardmäßige Therapie wurde die offene Appendektomie 1889 durch Charles McBurney eingeführt und prägte etwa 100 Jahre lang das operative Vorgehen (Reissfelder et al. 2009), bis 1983 durch den deutschen Gynäkologen Kurt Semm das Verfahren der laparoskopischen Appendektomie vorgestellt wurde, welches in der Folgezeit breite Anwendung erfuhr (Semm 1983).

\subsection{Epidemiologie}

Mit einem maximalen Manifestationsgipfel im 10. bis 19. Lebensjahr (Männer 10. bis 14. Lebensjahr, Frauen 15. bis 19. Lebensjahr) präsentiert sich die Appendizitis als eine Erkrankung des jungen Menschen (Addiss et al. 1990; Sulu et al. 2010), wobei in Studien ein häufigeres Auftreten bei Männern als bei Frauen (Verhältnis 1,4:1) beschrieben wird (Addiss et al. 1990; Al-Omran et al. 2003). Die Inzidenz dieser Erkrankung liegt in Europa und Nordamerika bei 100 Erkrankungen je 100.000 Einwohnern pro Jahr (Ohmann et al. 2002), jedoch ist die Appendektomierate mit jährlich etwa 135.000 durchgeführten chirurgischen Entfernungen der Appendix deutlich höher (Sahm et al. 2011). Analog wird das Lebenszeitrisiko für eine Appendizitis mit 8,6 \% bei Männern und 6,7 \% bei Frauen angegeben, während das Risiko für eine Appendektomie für 
Männer 12 \% und für Frauen 23 \% beträgt (Addiss et al. 1990). Übereinstimmend ist in der Literatur eine Abnahme der Inzidenz der nicht-perforierten Appendizitis bis etwa 1990 beschrieben (Ohmann et al. 2002). Nach diesem Zeitpunkt ist die Studienlage weniger eindeutig. Während einige Autoren eine Stabilisierung der Inzidenz der akuten, unkomplizierten Appendizitis verzeichnen (Körner et al. 2001; Ohmann et al. 2002), beschreiben andere ein weiteres Absinken der Inzidenz auch nach 1990 (Al-Omran et al. 2003; Andreu-Ballester et al. 2009). Einige Studien belegen sogar einen erneuten leichten Inzidenzanstieg (Livingston et al. 2007; Anderson et al. 2012). Davon abgesehen bleibt die Appendektomierate jedoch dauerhaft weit über der Inzidenz der akuten Appendizitis (Ohmann et al. 2002).

Unabhängig von der epidemiologischen Entwicklung der nicht-perforierten Appendizitis zeigt die fortgeschrittene, perforierte Appendizitis einen anderen Verlauf. Ihre Inzidenz wird mit etwa 20 Erkrankungen je 100.000 Einwohner pro Jahr als konstant angesehen (Ohmann et al. 2002). Der Anteil der schwerwiegenden Verlaufsformen mit Perforation, Abszessbildung oder generalisierter Peritonitis an allen akuten Appendizitiden liegt nach Addiss et al. bei Männern mit 19,2 \% etwas höher als bei Frauen mit 17,8 \% (Addiss et al. 1990) und auch andere Autoren beziffern die Wahrscheinlichkeit einer Perforation bei akuter Appendizitis mit etwa 20 \% (Zielke 2002). Während der Altersgipfel der unkomplizierten Appendizitis im 10. bis 19. Lebensjahr zu finden ist, haben insbesondere Kinder unter 5 Jahren und ältere Patienten über 65 Jahre ein erhöhtes Perforationsrisiko (Addiss et al. 1990; Treutner und Schumpelick 1997).

Um die beschriebenen Komplikationen zu vermeiden, wird eine zeitnahe Operation von Patienten mit typischen Symptomen einer akuten Appendizitis empfohlen. Weitgefasste Indikationsstellungen resultieren jedoch in einer hohen negativen Appendektomierate von bis zu $25 \%$ (Zielke 2002). Ihre Inzidenz zeigt dabei eine Geschlechtsabhängigkeit. Die negative Appendektomierate von Frauen im gebärfähigen Alter liegt etwa doppelt so hoch wie bei Männern desselben Alters (Addiss et al. 1990; Stein et al. 2012).

Zahlreiche Risikofaktoren werden als bedeutend für die Entstehung einer akuten Appendizitis angesehen. Hierzu zählen das männliche Geschlecht, eine weiße Hautfarbe und ein Lebensalter zwischen 10 und 19 Jahren (Ohmann et al. 2002). Eine jahreszeitliche Abhängigkeit wird in einigen Studien beschrieben, in welchen ein gehäuftes Auftreten von Appendizitiden während der Sommermonate vermerkt ist (Al-Omran et al. 2003; Anderson et al. 2012) und auch die Möglichkeit einer familiären Disposition der akuten Appendizitis wird diskutiert (Gauderer et al. 2001). 


\subsection{Anatomie und Pathogenese}

Die Appendix vermiformis entspringt als Ausstülpung des Zökums an der sternförmigen Vereinigungsstelle der drei Tänien etwa 2,5 cm unterhalb der Ileozökalklappe und liegt in der rechten Fossa iliaca. Ihre Länge wird durchschnittlich mit $7 \mathrm{~cm}$ und ihr Durchmesser mit etwa 0,7 cm angegeben, wobei eine starke individuelle Variabilität existiert. Ein eigenes Mesenteriolum, in welchem die Arteria appendicularis verläuft, gewährleistet die freie Beweglichkeit der Appendix innerhalb der Bauchhöhle und bedingt ihre vielfältigen Lagevarianten (Becker und Höfler 2002).

In etwa zwei Drittel der Fälle (65 \%) befindet sie sich in einer retrozökal-kranialen Position, in 30 \% liegt sie frei beweglich kaudal des Zökalpols mit Kontakt zu den Organen des kleinen Beckens. Weitaus seltener anzutreffende Positionen der Appendix sind die mediale (in Lagebeziehung zum Ileum), die laterale (zwischen seitlicher Bauchwand und Zökum) und die antezökale Lage. Die Appendixbasis projiziert sich als McBurney-Punkt auf die Bauchdecke, welcher in der Mitte einer gedachten Verbindungslinie zwischen Bauchnabel und rechter Spina iliaca anterior superior liegt (Peiper 2006). Die beschriebene Lagevariabilität ist verantwortlich für die vielgestaltige Symptomatik der akuten Appendizitis und erklärt die Schwierigkeiten, die sich in ihrer Diagnostik und der Indikationsstellung zur Operation ergeben können (Birnbaum und Wilson 2000).

Weitestgehend entspricht der histologische Wandaufbau dem des Kolons (Mukosa, Submukosa, Muskularis, Serosa) (Hollerweger 2006). Kennzeichnend sind jedoch zahlreiche Lymphfollikel, die innerhalb der Lamina propria mucosae und Submukosa zirkulär um das Appendixlumen angeordnet sind. Diese weisen auf die immunologischen Aufgaben der Appendix bei der Antigenerkennung und IgA-Produktion hin (Becker und Höfler 2002).

Der Beginn der entzündlichen Reaktion ist zumeist durch eine Obliteration des Appendixlumens bedingt. Für diese sind am häufigsten Koprolithen oder Schleim verantwortlich, doch können auch Nahrungsbestandteile, Tumoren, Narbengewebe, Schwellungen, lymphoide Hyperplasien oder Parasiten zu einer Stenose führen. Aus der fortschreitenden Sekretion von Mukosaschleim bei gleichzeitig behindertem Abtransport folgen ein intraluminaler Druckanstieg und ein gestörter venöser Blutfluss. Die eintretende Hypoxie stört die Barrierefunktion der Appendix-Mukosa und ermöglicht so das Eintreten von Bakterien (Birnbaum und Wilson 2000; Harrison und Benziger 2012). Anatomische Besonderheiten der Appendix begünstigen ihre Obstruktion und die Entstehung einer Entzündung. Hierzu zählen die Gerlach-Klappe an der Appendixbasis und die Wandstruktur, deren gitterartige Kollagenvernetzung eine Ausdehnung der Appendixlichtung erschwert und einen intraluminalen Druckanstieg begünstigt. Nicht unterschätzt werden darf weiterhin die arterielle Versorgung durch die Arteria appendicularis, die als 
funktionelle Endarterie einen entzündungsbedingt gesteigerten Sauerstoffbedarf nicht decken und so einen ischämischen Zelluntergang bedingen kann (Becker und Höfler 2002).

In der Entwicklung einer akuten Appendizitis werden unterschiedliche Krankheitsstadien durchlaufen, deren genaue Abgrenzung aufgrund ihres fließenden Übergangs nicht möglich ist. Im Stadium der katarrhalischen Appendizitis (Primäraffekt) mit vermehrter Serosagefäßzeichnung und oberflächlichen Schleimhautdefekten ist eine vollständige Ausheilung noch möglich. Schreitet die Erkrankung weiter voran, präsentiert sich die Appendix mit schmierig-grauen Fibrinbelägen und deutlicher Schwellung (phlegmonöse Appendizitis), später auch mit Schleimhautulzerationen (ulzerophlegmonöse Appendizitis). Bei beginnender Zellnekrose mit purulenten Einschmelzungsherden in allen Wandschichten ist das Stadium der abszedierten Appendizitis erreicht, gefolgt vom Zustand der gangränösen Appendizitis. Hier präsentiert sich der Wurmfortsatz durch fortgeschrittene Gewebenekrosen makroskopisch dunkelrot bis blauschwarz verfärbt. Grundsätzlich zeigt die Appendizitis Spontanheilungstendenzen, die jedoch nur im Stadium des Primäraffekts im Sinne einer Restitutio ad integrum verlaufen. Ausheilungen in späteren destruktiven Erkrankungsabschnitten gehen mit Defektheilungen und Narbenbildung einher, welche die Grundlage für weitere obliterationsbedingte Appendizitiden bilden können (Becker und Höfler 2002; Langner und Gabbert 2012).

Bei Ausbreitung der Entzündung über die Organgrenzen hinweg spricht man von einer Periappendizitis, die eine lokale Peritonitis nach sich ziehen kann. Durch Wandnekrosen kann es zur Perforation der Appendix vermiformis kommen. Infolge des Kontakts von infektiösem Appendixinhalt mit der Bauchhöhle bildet sich ein periappendikaler Abszess (perityphlitischer Abszess), der sich je nach Appendixlagevariante auch in einem Douglasabszess oder Schlingenabszess oder bei fortgeschrittenem Befund in einer generalisierten Peritonitis manifestieren kann (Birnbaum und Wilson 2000; Peiper 2006).

\subsection{Symptomatik}

Die gründliche Anamneseerhebung ist ein wesentlicher Bestandteil der Diagnosestellung der akuten Appendizitis. Innerhalb der Diagnostik hat sie zusammen mit dem klinischen Befund einen hohen Vorhersagewert (Lee et al. 2001). Obwohl die akute Appendizitis sehr variable Verläufe zeigt, welche die Herausforderungen der korrekten Diagnosestellung begründen, ist das Leitsymptom der abdominale Dauerschmerz im rechten Unterbauch (Isenmann et al. 2008).

Bei Beginn der Schmerzsymptomatik sind zunächst unspezifische kolikartige epigastrische und periumbilikale Beschwerden typisch (Humes und Simpson 2006). Diese viszeralen Schmerzen entstehen durch druckbedingte Dehnung der Appendix mit Reizung von afferenten Nervenfasern (Harrison und Benziger 2012). Innerhalb von Stunden kommt es durch die Einbeziehung 
des Peritoneum parietale in den Entzündungsprozess zu einer Qualitäts- und Lokalisationsverschiebung der Beschwerden. Sie entwickeln den Charakter eines gut lokalisierbaren und stechenden somatischen Dauerschmerzes im rechten Unterbauch (Peiper 2006). Begleitet wird dieser von unspezifischen Symptomen wie Appetitlosigkeit, Übelkeit, Erbrechen, Fieber und Stuhlverhalt, welche nach Schmerzbeginn einsetzen. Auch in der Folge auftretende Diarrhoen sind möglich (Isenmann et al. 2008). Eine lokale Peritonitis äußert sich in Perkussions- und Erschütterungsschmerz sowie lokaler Abwehrspannung im rechten Unterbauch bei Reizung, beispielsweise im Rahmen der klinischen Untersuchung (Humes und Simpson 2006). Schreitet die Entzündung weiter voran, ist eine Schonhaltung des Patienten, mit Anwinkeln der unteren Extremitäten und Einnahme einer vornübergebeugten Haltung, typisch. Ein plötzliches Nachlassen der Beschwerdesymptomatik kann hinweisend auf eine Perforation sein, die mit einer kurzzeitigen Entlastung des intraluminalen Druckes des entzündeten Wurmfortsatzes einhergeht (Peiper 2006).

Nicht immer zeigt die Symptomatik der akuten Appendizitis einen so typischen Verlauf. Vor allem bei jungen Kindern und älteren Menschen werden häufig atypische Krankheitsentwicklungen beschrieben (Zielke 2002). Auch das Punctum maximum des Schmerzes kann durch die individuell unterschiedliche Lage der Appendix vermiformis variieren, je nachdem wie schnell das anteriore Peritoneum parietale in den Entzündungsprozess einbezogen wird und die typischen rechtsseitigen Unterbauchschmerzen hervorruft (Peiper 2006).

\subsection{Diagnostik}

Bei Vorstellung eines Patienten mit aus der Anamnese hervorgehender Verdachtsdiagnose einer akuten Appendizitis wird zunächst eine klinische Untersuchung vorgenommen. Diese wird durch Laboruntersuchungen und gegebenenfalls bildgebende Verfahren ergänzt, um die Diagnose der akuten Appendizitis zu erhärten.

\subsubsection{Klinische Untersuchung}

Der klinischen Untersuchung kommt in Zusammenschau mit der speziellen Anamnese die größte Bedeutung in der Diagnostik der akuten Appendizitis zu (Lee et al. 2001). In Rückenlage des Patienten erfolgt eine Inspektion und Palpation des Abdomens. Neben der systematischen Überprüfung der in Tabelle 1 beschriebenen typischen Schmerzzeichen wird auf eventuell vorhandene Abwehrspannung und Peritonismus geachtet (Kreis et al. 2007). Während die rektale Untersuchung in einigen Veröffentlichungen als obligat bezeichnet wird (Mirow und Schiedeck 2008; Laurell et al. 2013), wird ihr diagnostischer Mehrwert in anderen Publikationen angezweifelt (Dixon et al. 1991; Petroianu 2012). 


\begin{tabular}{|l|l|}
\hline McBurney-Zeichen & $\begin{array}{l}\text { Schmerzhafter Druckpunkt in der Mitte der Verbindung zwischen Na- } \\
\text { bel und rechter Spina iliaca anterior superior }\end{array}$ \\
\hline Lanz-Zeichen & $\begin{array}{l}\text { Schmerzhafter Druckpunkt zwischen äußerem und mittlerem Drittel } \\
\text { rechts auf der Verbindungslinie zwischen rechter und linker Spina ili- } \\
\text { aca anterior superior }\end{array}$ \\
\hline Blumberg-Zeichen & $\begin{array}{l}\text { Schmerzen im rechten Unterbauch bei Druck auf linken Unterbauch mit } \\
\text { plötzlichem Loslassen }\end{array}$ \\
\hline Rovsing-Zeichen & $\begin{array}{l}\text { Schmerzen im rechten Unterbauch bei retrogradem Ausstreichen des } \\
\text { Colons }\end{array}$ \\
\hline Douglasschmerz & $\begin{array}{l}\text { Schmerzen im rechten Unterbauch bei Anheben des rechten Beins ge- } \\
\text { gen einen Widerstand } \\
\text { Rechtsseitiger Schmerz bei digital-rektaler Untersuchung }\end{array}$ \\
\hline
\end{tabular}

Tabelle 1: Typische Schmerzzeichen bei akuter Appendizitis (Isenmann et al. 2008; Mirow und Schiedeck 2008).

\subsubsection{Laborchemische Untersuchung}

Der Nutzen einer laborchemischen Blutuntersuchung bei Verdacht auf Appendizitis ist umstritten. Da sie im Rahmen der Standarddiagnostik jedoch leicht durchzuführen ist, sehen einige Autoren sie als wichtiges Diagnostikum (Andersson 2004; Bates et al. 2014), während in anderen Publikationen ihr geringer Aussagewert kritisiert wird (Zielke 2002). Da kein spezifischer Appendizitismarker existiert (Al-Abed et al. 2014), dient die Erhebung der unspezifischen Entzündungsparameter „Leukozytenanzahl“ und „CRP“ in der Regel weniger der unmittelbaren Diagnosestellung als vielmehr der Einschätzung des inflammatorischen Schweregrades und dem Ausschluss von Differentialdiagnosen (Zielke 2002).

Die hohe Sensitivität einer Leukozytose (Leukozytenzahl > $10 \times 10^{9} / \mathrm{l}$ ) bei gleichzeitig niedriger Spezifität kann zu einer Überschätzung des Entzündungsschweregrades führen (Zielke 2002). In mehreren Studien wird der Aussagegehalt einer Leukozytenerhöhung in der allgemeinen Verdeutlichung einer ablaufenden Entzündungsreaktion gesehen. Da der Anstieg der Leukozytenanzahl aber nicht linear mit fortschreitender Entzündung verläuft und auch bei komplexem Entzündungszustand eine Stagnation der Leukozytenzahl beobachtet wurde (Grönroos und Grönroos 1999; Bates et al. 2014), ist ihre Aussage hinsichtlich des inflammatorischen Schweregrades eingeschränkt. Normale Leukozytenzahlen sind allerdings mit hohen negativen Appendektomieraten vergesellschaftet (Grönroos und Grönroos 1999). Innerhalb von 6 bis 12 Stunden findet bei ablaufender Entzündungsreaktion ein CRP-Anstieg statt. Im Gegensatz zu einer Leukozytose scheint die CRP-Erhöhung aber mit dem Schweregrad der Appendizitis (Perforation) zu korrelieren (Chung et al. 1996; Grönroos und Grönroos 1999; Sack et al. 2006). Die höchste Sen- 
sitivität bei der Diagnostik der akuten Appendizitis hat eine Erhöhung beider Entzündungsparameter (Andersson 2004).

\subsubsection{Bildgebende Verfahren}

Eine wichtige Säule der Diagnostik der akuten Appendizitis stellt die abdominale Ultraschalluntersuchung dar. Aufgrund der kostengünstigen Durchführung, Wiederholbarkeit und Nebenwirkungsfreiheit ist sie das bildgebende Verfahren „der ersten Wahl“ (Binnebösel et al. 2009, S. 579) und wird zur Einschätzung des Entzündungsfortschritts und zum Ausschluss möglicher Differentialdiagnosen herangezogen. Weitreichende Verbesserungen der Detaildarstellung von Ultraschallgeräten in den letzten Jahrzehnten ermöglichen heutzutage auch die Darstellung der gesunden Appendix (Hollerweger 2006). Diese präsentiert sich als tubuläre, kompressible Struktur mit einem Durchmesser unter $6 \mathrm{~mm}$ und deutlich erkennbarer histologischer Wandschichtung (Binnebösel et al. 2009). Fehlende Wandstruktur und durch intraluminale Flüssigkeitsansammlung hervorgerufene schlechte Kompressibilität sind Indizien eines pathologischen Appendixzustandes (Kessler et al. 2004; Binnebösel et al. 2009). Hohe Sensitivität kann der Nachweis eines Appendixdurchmessers von mehr als 6 mm erreichen (Manner und Stickel 2001; Rettenbacher et al. 2001; Kessler et al. 2004). Von hohem diagnostischem Wert ist des Weiteren die Darstellung einer pathologischen Kokarde (Franke et al. 1999), deren „Zielscheibenmuster“ durch Echogenitätsunterschiede der geschwollenen Wandschichten im fortgeschrittenen Entzündungsstadium hervorgerufen wird (Binnebösel et al. 2009). Freie periappendikale Flüssigkeit, Lymphknotenschwellung und verminderte Peristaltik sind weniger spezifische Appendizitiszeichen, weisen jedoch auf einen allgemein pathologischen Prozess hin (Peiper 2006). Anhand der charakteristischen sonographischen Befunde ist eine Abgrenzung der unterschiedlichen Appendizitisstadien möglich, bleibt jedoch mit Unsicherheiten behaftet (Zielke 2002). Auch eine Abszessdarstellung gelingt in vielen Fällen (Binnebösel et al. 2009). Nachteile der Sonographie sind Untersucherabhängigkeit (Zielke et al. 2001) und erschwerte Befundung bei Darmgasüberlagerung, Adipositas und Schmerzen des Patienten (Galindo Gallego et al. 1998). Hinsichtlich der diagnostischen Genauigkeit der Sonographie herrscht in der Literatur Uneinigkeit. Sowohl Sensitivität als auch Spezifität zeigen große Spannweiten von 46 bis $98 \%$ beziehungsweise 93 bis 100 \% (Sahm et al. 2011). Zusammenfassend zeichnet sich aber der Trend einer höheren Spezifität der sonographischen Appendizitiszeichen im Vergleich zur Sensitivität ab, woraus sich eine Empfehlung zur Operation bei sonographisch pathologischem Befund ableitet (Zielke 2002).

Als weitere Methode der diagnostischen Bildgebung zeigt die computertomographische Untersuchung diagnostische Genauigkeitswerte von 90 bis $100 \%$ (Sensitivität) und 91 bis $99 \%$ (Spezifität) (Birnbaum und Wilson 2000). Beim Vergleich zwischen CT und Sonographie ist eine 
Überlegenheit der Sensitivität und Spezifität der CT-Untersuchung (Doria et al. 2006; van Randen et al. 2008) - bei geringerer Abhängigkeit der Befundung vom Erfahrungsstand des Behandlers und Unabhängigkeit der Bildqualität von Faktoren wie Adipositas oder Darmvergasung des Patienten - erkennbar (Sahm et al. 2011). Aus Gründen der Strahlenhygiene, der eingeschränkten Verfügbarkeit und der kostenintensiven Diagnostik wird die CT-Untersuchung im deutschsprachigen Raum in der Routinediagnostik der akuten Appendizitis trotzdem nur selten eingesetzt (Peiper 2006).

\subsubsection{Differentialdiagnosen}

Der beschriebene „klassische“ Krankheitsverlauf tritt nur in etwa 50 bis $60 \%$ der akuten Appendizitiden auf (Birnbaum und Wilson 2000). Bei untypischer Symptomatik sind einerseits erweiterte diagnostische Maßnahmen indiziert und andererseits differentialdiagnostische Überlegungen anzustellen. Da das Krankheitsbild des akuten Abdomens neben der akuten Appendizitis auch durch andere Krankheitszustände hervorgerufen werden kann, sind mannigfaltige Differentialdiagnosen denkbar (Isenmann et al. 2008). Die folgende Auflistung erhebt daher keinen Anspruch auf Vollständigkeit, sondern betrachtet lediglich klinisch häufig auftretende Differentialdiagnosen.

Andere gastroenterologische Erkrankungen wie Gastroenteritis, Lymphadenitis mesenterialis, Morbus Crohn, Ileus, Divertikulitis oder Kolonkarzinome können ähnliche Symptome wie eine akute Appendizitis hervorrufen. Die bereits beschriebene höhere negative Appendektomierate bei Frauen im Vergleich zu Männern lässt auf einen hohen Stellenwert von gynäkologischen Erkrankungen innerhalb der Differentialdiagnostik schließen. Hier sind insbesondere eine Adnexitis, eine stielgedrehte oder rupturierte Ovarialzyste sowie eine extrauterine Schwangerschaft zu nennen. Auch urologische Erkrankungen sollten bei Verdacht auf eine akute Appendizitis ausgeschlossen werden. Hierzu zählen Hodentorsion, Entzündungen des Nierenbeckens und der ableitenden Harnwege (Pyelonephritis, Zystitis) und Verlegung selbiger durch Harnsteine oder Tumoren. Auch neurologische oder orthopädische Erkrankungen wie Koxarthrose oder Bandscheibenprolaps sollten differentialdiagnostisch in Erwägung gezogen werden (Kreis et al. 2007; Isenmann et al. 2008).

\subsubsection{Operationsindikation}

Die Verdachtsdiagnose einer akuten Appendizitis wird als Indikation zur zeitnahen chirurgischen Therapie angesehen (Peiper 2006). Während jedoch bei klassischer Befundkonstellation sogar ein Verzicht auf weiterführende Diagnostik erwogen werden kann (Kreis et al. 2007), bleibt der Ausschluss einer akuten Appendizitis bei unklaren Befunden eine Herausforderung (Manner und Stickel 2001). Hohe Perforationsraten bei kleinen Kindern und alten Patienten 
erfordern in diesen Altersgruppen großzügige Indikationsstellungen, da die klinische Symptomatik bekanntermaßen häufig atypisch verläuft (Peiper 2006). Allgemein großzügige Operationsindikationen korrelieren allerdings mit hohen negativen Appendektomieraten (Sahm et al. 2011), bei denen der Patient unnötigen operativen Risiken ausgesetzt wird. Dieses Dilemma begründet die Suche vieler Autoren nach eindeutigen Indikatoren, die mit hoher Genauigkeit die Notwendigkeit einer operativen Intervention vorhersagen können. Zahlreiche Bestrebungen durch Entwicklung von Scores die Treffsicherheit der Indikationsstellung zu erhöhen, haben im klinischen Alltag jedoch keine Verbreitung gefunden (Wente und Waleczek 2009). Die klinische Beurteilung des Patienten bleibt - trotz des rasanten Fortschrittes in der Diagnostik durch Einführung und Verbesserung von bildgebenden Verfahren - das Mittel der Wahl zur Diagnosestellung der akuten Appendizitis. Nach wie vor ist demnach die Erfahrung des behandelnden Arztes unverzichtbar, um den Verlauf dieser Erkrankung einschätzen und eine korrekte Operationsindikation stellen zu können (Sahm et al. 2011).

\subsection{Therapie und medizinisches Ergebnis}

Die Therapie bei Diagnose einer akuten Appendizitis erfolgt chirurgisch. Kann eine Appendizitis nicht sicher ausgeschlossen werden, so erfolgt in der Regel eine operative Intervention, da in der Abwägung des Chirurgen die potentiellen Gefahren postoperativer Komplikationen im Zusammenhang mit einer „Verschleppung“ des Entzündungszustandes gegenüber den Operationsrisiken überwiegen (Mirow und Schiedeck 2008). Mit einer Mortalitätsrate von unter $1 \%$ stellt die Appendektomie eine vergleichsweise sichere Operation dar (Peiper 2006). Bezüglich des chirurgischen Vorgehens trifft der Operateur die Wahl zwischen konventioneller Laparotomie und minimalinvasiver Laparoskopie, wobei die Entscheidungsfindung von verschiedenen Faktoren abhängt. Junges Alter, weibliches Geschlecht, geringe laborchemische Entzündungsparameter und leichter klinischer Schweregrad sowie individuelle Erfahrung des Chirurgen und Tageszeit sind mit einer Entscheidung zur Laparoskopie vergesellschaftet (Horstmann et al. 2005). Beide Operationsverfahren sind dabei heute als gleichwertig anzusehen (Lippert et al. 2002; Magdeburg und Kähler 2013). In Deutschland ist in den letzten Jahren der Anteil der laparoskopisch durchgeführten Operationen kontinuierlich angestiegen (Reissfelder et al. 2009; Sahm et al. 2013). International ist dieser Trend jedoch nicht nachweisbar (Sahm et al. 2011).

Nach ihrer Erstbeschreibung durch Kurt Semm (Semm 1983) wurden schwerwiegende Komplikationen im Zusammenhang mit laparoskopischen Appendektomien beschrieben wie vermehrte intraabdominelle Abszessbildung und insuffiziente Appendixstumpfverschlüsse. Ein erhöhtes Risiko für derartige Komplikationen ist heute jedoch nicht mehr erkennbar (Faiz et al. 2008; Sahm et al. 2011). Die Vorteile der Laparoskopie liegen in ihrer Minimalinvasivität, welche 
mit einer kürzeren Liegedauer, niedrigerer Wundinfektionsrate, geringeren gastrointestinalen Komplikationen und schnellerer Regeneration verbunden ist (Lippert et al. 2002; Guller et al. 2004). Aufgrund der möglichen Operationserweiterung im Sinne einer diagnostischen Laparoskopie profitieren insbesondere Frauen im gebärfähigen Alter und Patienten mit Adipositas von einem laparoskopischen Vorgehen (Lippert et al. 2002). Nachteilig ist die etwas längere Operationsdauer im Vergleich zur konventionellen Appendektomie (Lippert et al. 2002; Horstmann et al. 2005). Vermieden wird eine laparoskopische Appendektomie in der Regel bei präoperativer Verdachtsdiagnose einer Appendixperforation, eines perityphlitischen Abszesses oder bei starken abdominellen Verwachsungen. In diesen Fällen wird meist direkt ein offenes Vorgehen angestrebt, welches den Vorteil eines leichteren operativen Umstiegs durch Schnitterweiterung bietet (Isenmann et al. 2008). Zahlreiche Studien wurden in den letzten Jahren zum Vergleich des offenen und laparoskopischen Vorgehens veröffentlicht, konnten jedoch keine klare Überlegenheit einer Operationsmethode darlegen.

Standardmäßig wird mittlerweile eine perioperative Antibiotikaprophylaxe durchgeführt, da ein positiver Einfluss auf die postoperative Wundinfektionsrate nachgewiesen werden konnte. Eine antibiotische Therapie in Kombination mit chirurgischer Intervention erfolgt allerdings nur bei Vorliegen einer Peritonitis oder Abszessbildung (Koch et al. 2000). Die konservative Therapie der akuten Appendizitis mit alleiniger antibiotischer Abschirmung des Patienten wird ebenfalls diskutiert, konnte sich aber bis heute nicht durchsetzen. Zahlreiche nach Therapieabschluss notwendige Operationen durch Remanifestation einer akuten Appendizitis scheinen den Befürwortern einer sofortigen chirurgischen Lösung Recht zu geben (Magdeburg und Kähler 2013).

Innerhalb von 24 Stunden nach Operation erfolgt in der Regel der postoperative Kostaufbau. Nach etwa 2 bis 3 Tagen kann der Patient normalerweise bei komplikationslosem Verlauf entlassen werden. Typische postoperative Komplikationen sind Wundheilungsstörungen, Ileus und durch intraabdominell verbliebenes infektiöses Material oder unzureichende Appendixstumpfversorgung hervorgerufene Abszessbildung. Je nach Schweregrad kann eine chirurgische Revision, verbunden mit Abdomenlavage und Drainageeinlage, notwendig werden (Peiper 2006). 


\subsection{Fragestellung und Zielsetzung der Arbeit}

Anhand der retrospektiven Untersuchung eines Patientenkollektivs, welches mit der Verdachtsdiagnose einer akuten Appendizitis operiert wurde, sollen Rückschlüsse auf die Verlässlichkeit der präoperativen Diagnostik, auf das chirurgische Management der Erkrankung und den Stellenwert der intraoperativen Beurteilung der akuten Appendizitis gezogen werden.

Folgende Fragestellungen werden im Einzelnen bearbeitet:

Können präoperative Prädiktoren das Vorliegen einer akuten Appendizitis zuverlässig vorhersagen? Kann ein komplikationsbehafteter Krankheitsverlauf anhand präoperativer Prädiktoren abgeschätzt werden?

Kann durch frühzeitige Operation die Rate an fortgeschrittenen Appendizitiden gesenkt werden? Welche Auswirkungen hat das Vorliegen einer Perforation oder Peritonitis auf den postoperativen Heilungsverlauf?

Inwieweit stimmen Chirurg und Pathologe in ihrer Einschätzung des Entzündungsschweregrades überein? 


\section{Patienten und Methoden}

\subsection{Datenerhebung und statistische Auswertung}

In die vorliegende retrospektive Arbeit flossen Daten aller Patienten ein, die im Zeitraum vom 01.01.2008 bis 30.04.2013 in der Klinik für Allgemein-, Viszeral- und Kinderchirurgie der Universitätsmedizin Göttingen unter der Verdachtsdiagnose einer akuten Appendizitis appendektomiert wurden. Die Genehmigung zur Durchführung dieser Studie erfolgte durch die Ethikkommission der Universitätsmedizin Göttingen, Antragsnummer: DOK_104_2015.

Anhand der ICD-10 K35.- (akute Appendizitis) beziehungsweise des OPS-Codes 5-470.(Appendektomie) wurden alle im genannten Zeitraum operierten Patienten aus dem KlinikInformationssystem ermittelt. Im Rahmen der Datenerhebung wurden nachfolgend alle Patienten mit einer Gelegenheitsappendektomie und Operation ohne Verdachtsdiagnose einer akuten Appendizitis ausgeschlossen.

Alle verfügbaren papierbasierten und elektronischen Patientenakten wurden eingesehen und zur Datensammlung herangezogen. Hierbei ließen partielle Unvollständigkeiten in der Aktendokumentation in einigen wenigen Fällen eine nur unvollständige Datenerhebung für einzelne Parameter zu. Die Erfassung der Daten erfolgte in einer Excel-Tabelle (Microsoft Excel 2013) und die anschließende statistische Analyse sowie die Erstellung der im Ergebnisteil präsentierten Diagramme unter Verwendung der Statistik-Software SPSS 21 von IBM. Die statistische Auswertung der erhobenen Daten erfolgte im Falle von qualitativen Merkmalen mithilfe von Kontingenztabellen unter Verwendung von exakten Fisher-Tests und Chi-Quadrat-Tests. Der statistische Gruppenvergleich im Falle von quantitativen Merkmalen erfolgte mittels $\mathrm{t}$-Tests beziehungsweise Mann-Whitney-U-Tests. Das Signifikanzniveau wurde auf $\mathrm{p}<0,05$ festgelegt.

\subsection{Parameter}

\subsubsection{Demographische Daten}

Aus den Patientenakten wurden Geschlecht, Alter, Gewicht, Größe und BMI zum Zeitpunkt der stationären Aufnahme ermittelt. 


\subsubsection{Anamnestische Daten}

Daten zur präoperativen Symptomatik wurden anhand des Aufnahmeuntersuchungsbogens der allgemeinchirurgischen oder pädiatrischen Abteilung erhoben. Die vom Patienten angegebene Schmerzdauer wurde auf ganze Stunden gerundet. Gab der Patient eine Uhrzeit an, seit der die Schmerzen bestanden, so wurde anhand der vermerkten Aufnahme-Uhrzeit die Stundenanzahl errechnet.

Daten zur Schmerzqualität und zum gastrointestinalen Befund wurden nur bei Angaben im Aufnahmebogen erhoben. Erfragt wurde kolikartiger Schmerz, Dauerschmerz und Schmerzwanderung sowie Übelkeit, Erbrechen oder Diarrhoe des Patienten. Mehrfachnennungen waren hierbei möglich.

\subsubsection{Untersuchungsbefund und apparative Diagnostik}

Anhand des Aufnahmebogens wurde die durchgeführte Patientenuntersuchung ausgewertet. Besonderes Augenmerk lag hierbei auf der Dokumentation typischer Appendizitis-Zeichen: Druckschmerz im rechten Unterbauch, McBurney- und Lanz-Zeichen, kontralateraler Loslassschmerz (Blumberg-Zeichen) sowie Psoasschmerz. Andere Appendizitis-Zeichen wie RovsingZeichen und Douglasschmerz wurden an der Universitätsmedizin Göttingen nicht standardmäßig überprüft und flossen aus diesem Grund nicht in die Datenerhebung ein. Bewertet wurde außerdem eine aufgetretene Abwehrspannung sowie andere Zeichen von Peritonismus wie Klopf- und Erschütterungsschmerz. Des Weiteren wurden auch allgemeine Symptome eines akuten Abdomens in die Datenauswertung einbezogen wie Druckschmerz im rechten Oberbauch, im linken Unterbauch sowie periumbilikaler und epigastrischer Druckschmerz. Bei Unvollständigkeit des chirurgischen Untersuchungsbefundes wurden nicht genannte Zeichen als negativ bewertet und im Falle mehrfacher Nennungen flossen alle dokumentierten Zeichen in die Datenerhebung ein.

Neben der körperlichen Untersuchung waren auch Aspekte der bildgebenden Diagnostik in Form von sonographischer und computertomographischer Untersuchung von Interesse. Bei der Ultraschalluntersuchung wurde anhand des dokumentierten sonographischen Befundes die Darstellung einer unauffälligen Appendix, der Nachweis von freier Flüssigkeit oder einer pathologischen Kokarde, von freier Flüssigkeit und Kokarde sowie eines perityphlitischen Abszesses erfasst. Des Weiteren wurde die Durchführung einer CT-Untersuchung dokumentiert. In die Datenerfassung flossen dabei anhand des CT-Untersuchungsbefundes der Nachweis einer akuten Appendizitis oder eines sonstigen radiologisch erkennbaren pathologischen Befundes ein, wobei in einigen Fällen sowohl Appendizitis als auch andere pathologische Befunde genannt und der Datenerhebung zugeführt wurden. 
Im Rahmen der standardmäßigen Aufnahmeuntersuchung wurde die axilläre Körpertemperatur gemessen. Des Weiteren floss die erste Blutuntersuchung nach stationärer Aufnahme in die Datenerhebung ein. Als laborchemische Entzündungsmarker dienten Leukozytenanzahl und CRP. Von einem pathologischen CRP-Anstieg wurde bei mehr als $5 \mathrm{mg} / \mathrm{l}$ ausgegangen. Eine Leukozytose wurde als Leukozytenanzahl oberhalb von 10 x 109/l definiert. Wurden Leukozytenanzahl und CRP bei mangelnder Aktendokumentation im Entlassschreiben des behandelnden Arztes als „Norm“ bezeichnet, so wurde bei der Datenerhebung von einer Leukozytenanzahl von $10 \times 10^{9} / \mathrm{l}$ und von einem CRP von $2,5 \mathrm{mg} / \mathrm{l}$ ausgegangen.

\subsubsection{Perioperative Daten}

Perioperative Daten wurden aus OP-Bericht und OP-Protokoll entnommen. Aus der klinikumsinternen elektronischen Patientenerfassung und der OP-Ablaufsteuerung konnte sowohl die genaue Uhrzeit der Patientenaufnahme als auch der genaue Operationsbeginn (Schnittzeit) ermittelt werden. Anhand dieser Daten war die Errechnung der Zeit zwischen Aufnahme und Schnitt möglich.

Dem OP-Bericht war weiterhin die Operationsdauer, der operative Zugang (offen, laparoskopisch oder konvertiert), eine erfolgte Drainageeinlage und eine eventuell durchgeführte Erweiterung der Operation zu entnehmen. Als OP-Erweiterung wurde jede operative Maßnahme gewertet, die nicht im unmittelbaren Zusammenhang mit der Entfernung der Appendix vermiformis stand. Im Falle eines offenen oder konvertierten Vorgehens wurde die Schnittführung und im Falle einer Laparoskopie die Methodik der Absetzung der Appendix mittels Stapler oder Schlinge erfasst.

\subsubsection{Intraoperativer Befund}

Der intraoperative Zustand der Appendix wurde dem OP-Bericht entnommen. Von Interesse war eine Beschreibung der Appendix als blande oder akut entzündet. Im Falle einer genaueren Einstufung des Entzündungsgrades wurden die Stadien „fibrinös“, „ulcero-phlegmonös“, „abszediert“, „gangränös“ und „perforiert“ unterschieden, wobei hier Mehrfachnennungen möglich waren. Die Diagnose einer akuten Appendizitis wurde bei der Datenerhebung nur verwendet, wenn keine weiterführende Einschätzung des Entzündungsschwergrades aufgeführt war („nicht näher definiert akut").

Als intraoperativer Zusatzbefund wurden Beschreibungen von Peritonitis, trüber Flüssigkeit und eines perityphlitischen Abszesses in die Datenerhebung aufgenommen. 


\subsubsection{Histologischer Befund}

Analog zur intraoperativen Beschreibung der Appendix erfolgte die Interpretation des histologischen Befundes. Eine Appendizitis wurde anhand der Stadien „katarrhalisch“, „ulcerophlegmonös“, „abszediert“, „gangränös“ und „perforiert“ klassifiziert, wobei auch hier Mehrfachnennungen möglich waren. Als blande wurde die Appendix bewertet, wenn im histologischen Befund eine reizlose Appendix, eine Appendix mit Vernarbung oder Zeichen einer chronischen Entzündung erwähnt wurden. Nur bei ausschließlicher Benennung der Appendix als akut entzündet wurde der Schweregrad der Appendizitis als „nicht näher definiert akut“ vermerkt.

Im Rahmen der Erhebung von histologischen Zusatzbefunden wurde die Beschreibung einer Periappendizitis, Peritonitis und einer Koprostase in die Datentabelle aufgenommen.

\subsubsection{Postoperative Daten}

Der postoperative Aufenthalt sowie die Gesamtverweildauer wurden mit Hilfe der klinikumsinternen Software zur elektronischen Patientenverwaltung errechnet.

Auftretende postoperative Komplikationen wurden anhand des Entlassbriefes verzeichnet. Hierbei waren Mehrfachnennungen möglich. Besonderes Augenmerk wurde auf Wundheilungsstörungen, intraabdominelle Abszesse, Revisionsoperationen und Re-Hospitalisationen gelegt. Andere auftretende Komplikationen wie Ileus, Fieber, Anstieg der Entzündungsparameter, Sepsis, Darmperforationen, Drainagenverlagerung und Hernienbildung wurden unter „Sonstige“ zusammengefasst.

\subsection{Subgruppenanalyse}

Die genaue Untersuchung der in Kapitel 1.7 formulierten Fragestellungen erfolgte durch Subgruppenanalysen. Hierbei wurde das Gesamtkollektiv in definierte Untergruppen unterteilt, deren Vergleich Unterschiede des jeweiligen prä-, peri- und postoperativen Verlaufs aufzeigen sollte.

Es erfolgte die Subgruppenunterteilung nach dem Gesichtspunkt der Diagnose einer akuten oder blanden Appendix, einerseits im intraoperativen und andererseits im histologischen Befund. Zur Bearbeitung der Frage nach möglichen präoperativen Prädiktoren eines komplizierten postoperativen Genesungsverlaufs erfolgte die Aufgliederung des Gesamtkollektivs hinsichtlich der Beschreibung des stationären Aufenthaltes als kompliziert oder komplikationsfrei. Als Merkmale eines fortgeschrittenen Entzündungszustandes wurden die Parameter „Appendixperforation“ und „Peritonitis“ einer genauen Untersuchung unterzogen. Das Gesamtkollektiv wurde 
dabei entsprechend der intraoperativen Diagnose einer perforierten Appendizitis beziehungsweise einer Peritonitis in Untergruppen aufgeteilt.

\subsection{Gegenüberstellung intraoperativer und histologischer Befund}

Zur Überprüfung der Frage nach der Übereinstimmung von intraoperativem und histologischem Befund erfolgte zunächst die Untersuchung der chirurgischen und pathologischen Meinungskongruenz in Bezug auf die Appendixbeurteilung als akut entzündet oder reizlos und als perforiert oder nicht-perforiert.

Des Weiteren wurde auch der Übereinstimmungsgrad des intraoperativen und histologischen Befundes hinsichtlich des vorliegenden Appendizitisschweregrades mithilfe eines BlandAltmann-Plots ermittelt. Hierbei wurde den im Befund genannten Appendizitisstadien ein ordinaler Punktwert entsprechend Tabelle 2 zugeordnet. Bei Nennung mehrerer Schweregrade floss der jeweils höchste Wert in die Datenanalyse ein.

\begin{tabular}{|l|c|}
\hline \multicolumn{1}{|c|}{ Schweregrad der Appendizitis } & Punktwert \\
\hline reizlos & 1 \\
\hline nicht näher definiert akut & 2 \\
\hline fibrinös (im intraoperativen Befund) & 3 \\
katarrhalisch (im histologischen Befund) & 3 \\
\hline ulcero-phlegmonös & 4 \\
\hline abszediert & 5 \\
\hline gangränös & 6 \\
\hline perforiert & 7 \\
\hline
\end{tabular}

Tabelle 2: Ordinalskala des im histologischen oder intraoperativen Befund genannten Schweregrades der Appendizitis. 


\section{Ergebnisse}

\subsection{Gesamtkollektiv}

Im Zeitraum von Januar 2008 bis April 2013 wurden 694 Patienten in der Klinik für Allgemein-, Viszeral- und Kinderchirurgie der Universitätsmedizin Göttingen appendektomiert. Nach Ausschluss von Gelegenheitsappendektomien und Patienten, die nicht unter der präoperativen Verdachtsdiagnose einer akuten Appendizitis appendektomiert wurden, verblieb ein Gesamtkollektiv von 655 Patienten.

\subsubsection{Demographische Daten}

Wie Tabelle 3 zu entnehmen ist, waren Frauen innerhalb des Gesamtkollektivs mit etwa $52 \%$ etwas stärker vertreten als Männer mit 48 \%. Größtenteils gehörten die Patienten dem Personenkreis der jungen Erwachsenen und Kinder an und zeigten eine durchschnittliche körperliche Konstitution (Tabelle 3).

\begin{tabular}{|l|c|c|}
\hline \multicolumn{1}{|c|}{ Parameter } & Absolute Anzahl & Mittelwert \pm SD \\
\hline Geschlecht & $\mathbf{6 5 5}$ & \\
weiblich & 343 & \\
männlich & 312 & $28,4 \pm 19,2$ \\
\hline Alter [y] & $\mathbf{6 5 5}$ & $66,2 \pm 23,4$ \\
\hline Gewicht $[\mathrm{kg}]$ & $\mathbf{6 1 7}$ & $1,67 \pm 0,16$ \\
\hline Größe $[\mathrm{m}]$ & $\mathbf{5 5 6}$ & $23,9 \pm 5,8$ \\
\hline BMI $\left[\mathrm{kg} / \mathrm{m}^{2}\right]$ & $\mathbf{5 5 4}$ & \\
\hline
\end{tabular}

Tabelle 3: Demographische Daten des Gesamtkollektivs. Daten als Absolutwerte und Mittelwert \pm Standardabweichung.

Die Altersverteilung im Gesamtkollektiv zeigte einen Häufigkeitsgipfel zwischen dem 10. und 29. Lebensjahr: $56 \%$ der Operierten (365 Personen) fielen in diese Kategorie. Auffallend war eine beinahe gleichstarke Verteilung zwischen dem Kollektiv der 10-bis-19-Jährigen und dem der 20-bis-29-Jährigen (181 vs. 184 Personen). Ein lokaler Häufigkeitsgipfel war innerhalb der 20-bis-24-Jährigen (115 Personen) zu finden.

Die restlichen 290 Patienten des Gesamtkollektivs (44\%) verteilten sich auf 66 operierte Personen zwischen 0 bis 9 Jahren und 224 Patienten zwischen dem 30. und 97. Lebensjahr. Ab 
dem 30. Lebensjahr war ein kontinuierlicher Abfall in der Appendektomiehäufigkeit bis zum Ende des Lebens feststellbar (Abbildung 1).

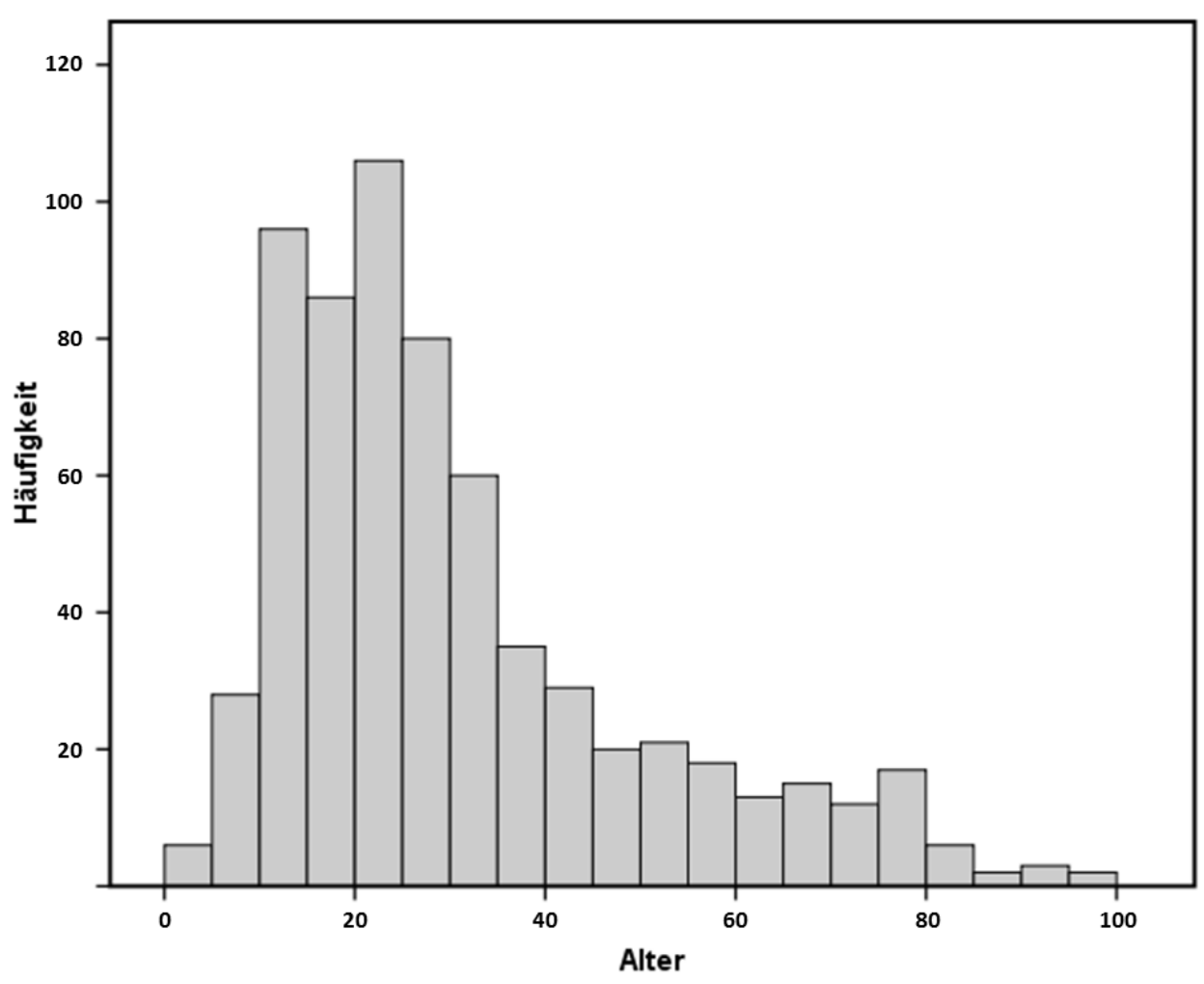

Abb. 1: Altersverteilung des Gesamtkollektivs. Die Balkenbreite entspricht Altersgruppen zu je 5 Jahren.

\subsubsection{Anamnestische Daten}

Wie in Tabelle 4 aufgeführt, stellten sich mehr als die Hälfte der Patienten mit länger als einen Tag andauernden abdominellen Beschwerden vor. Unter den Patienten mit kürzerer Beschwerdedauer trat diese zum großen Teil in einem mittleren Bereich von 4 bis 16 Stunden auf.

Auffallend war die Angabe eines abdominellen Dauerschmerzes, die fast $90 \%$ der Patienten tätigten. Eine für die akute Appendizitis typische Schmerzwanderung von der Periumbilikal- und epigastrischen Bauchregion in den rechten Unterbauch wurde deutlich seltener beschrieben und nur etwa jeder zehnte Patient beklagte kolikartige Bauchschmerzen.

Keiner der Parameter zur Bewertung des gastrointestinalen Beschwerdebildes der Patienten zeigte einen starken Häufigkeitsgipfel, auch wenn Übelkeit und Erbrechen etwas häufiger als Diarrhoe angegeben wurden (Tabelle 4). 


\begin{tabular}{|l|c|c|}
\hline \multicolumn{1}{|c|}{ Parameter } & Absolute Anzahl & Prozentualer Anteil \\
\hline Schmerzdauer & $\mathbf{5 8 9}$ & \\
Schmerzen $<4 \mathrm{~h}$ & 49 & $8,3 \%$ \\
Schmerzen 4 bis $<8 \mathrm{~h}$ & 79 & $13,4 \%$ \\
Schmerzen 8 bis $<16 \mathrm{~h}$ & 104 & $17,7 \%$ \\
Schmerzen 16 bis $<24 \mathrm{~h}$ & 55 & $9,3 \%$ \\
Schmerzen $\geq 24 \mathrm{~h}$ & 302 & $51,3 \%$ \\
Schmerzqualität & $\mathbf{6 4 2}$ & $11,5 \%$ \\
Kolik & 74 & $87,7 \%$ \\
Dauerschmerz & 563 & $20,4 \%$ \\
Schmerzwanderung & 131 & \\
\hline Gastrointestinaler Befund & $\mathbf{6 4 3}$ & $44,9 \%$ \\
Übelkeit & 289 & $31,4 \%$ \\
Erbrechen & 202 & $18,0 \%$ \\
Diarrhoe & 116 & \\
\hline
\end{tabular}

Tabelle 4: Anamnestische Daten des Gesamtkollektivs. Daten als Absolutwerte und prozentualer Anteil der untersuchten Kollektivgröße.

\subsubsection{Untersuchungsbefund und apparative Diagnostik}

\begin{tabular}{|l|c|c|}
\hline \multicolumn{1}{|c|}{ Parameter } & Absolute Anzahl & Prozentualer Anteil \\
\hline Druckschmerz & $\mathbf{6 4 0}$ & \\
rechter Unterbauch & 618 & $96,6 \%$ \\
linker Unterbauch & 137 & $21,4 \%$ \\
rechter Oberbauch & 63 & $9,8 \%$ \\
periumbilikal & 106 & $16,6 \%$ \\
epigastrisch & 61 & $9,5 \%$ \\
\hline Appendizitiszeichen & $\mathbf{6 4 0}$ & \\
McBurney-Zeichen & 208 & $32,5 \%$ \\
Lanz-Zeichen & 136 & $21,2 \%$ \\
Loslassschmerz & 217 & $33,9 \%$ \\
Psoasschmerz & 112 & $17,5 \%$ \\
Peritonismus & 101 & $15,8 \%$ \\
Abwehrspannung & 219 & $34,2 \%$ \\
\hline
\end{tabular}

Tabelle 5: Klinischer Untersuchungsbefund des Gesamtkollektivs. Daten als Absolutwerte und prozentualer Anteil der untersuchten Kollektivgröße.

Tabelle 5 ist zu entnehmen, dass im Rahmen der Aufnahmeuntersuchung auffallend häufig Druckschmerz im rechten Unterbauch dokumentiert wurde. Nur etwa 3 \% der Patienten hatten keine Beschwerden bei Palpation dieser Region. Jeder fünfte Patient äußerte zudem Druck- 
schmerz im linken Unterbauch, gefolgt von periumbilikalen Palpationsbeschwerden. Verhältnismäßig selten traten epigastrischer und Oberbauchdruckschmerz auf.

Keines der typischen Appendizitiszeichen wurde häufiger als an einem Drittel der Patienten nachgewiesen. Die Häufigkeiten eines positiven McBurney-Zeichens, von Loslassschmerz und Abwehrspannung waren ähnlich. Deutlich seltener erfolgte die Dokumentation eines LanzZeichens und der Nachweis von Psoasschmerz und Peritonismus (Tabelle 5).

\begin{tabular}{|c|c|c|c|}
\hline Parameter & Absolute Anzahl & Prozentualer Anteil & Mittelwert \pm SD \\
\hline Sonographie & 620 & & \\
\hline ohne pathologischen Befund & 350 & $56,5 \%$ & \\
\hline freie Flüssigkeit & 109 & $17,6 \%$ & \\
\hline Kokarde & 104 & $16,8 \%$ & \\
\hline freie Flüssigkeit und Kokarde & 43 & $6,9 \%$ & \\
\hline perityphlitischer Abszess & 14 & $2,3 \%$ & \\
\hline Computertomographie & 655 & & \\
\hline durchgeführt & 28 & $4,3 \%$ & \\
\hline nicht durchgeführt & 627 & $95,7 \%$ & \\
\hline akute Appendizitis & 20 & $71,4 \%$ & \\
\hline sonstiger radiologischer Befund & 18 & $64,3 \%$ & \\
\hline Temperatur $\left[{ }^{\circ} \mathrm{C}\right]$ & 396 & & $36,9 \pm 1,0$ \\
\hline Temperatur $\leq 37^{\circ} \mathrm{C}$ & 250 & $63,1 \%$ & \\
\hline Temperatur 37,1 bis $37,9^{\circ} \mathrm{C}$ & 81 & $20,5 \%$ & \\
\hline Temperatur $\geq 38^{\circ} \mathrm{C}$ & 65 & $16,4 \%$ & \\
\hline \multicolumn{4}{|l|}{ Blutuntersuchung } \\
\hline Leukozytenanzahl [x 109/l] & 647 & & $13,1 \pm 5,5$ \\
\hline $\mathrm{CRP}[\mathrm{mg} / \mathrm{l}]$ & 644 & & $40,3 \pm 62,2$ \\
\hline Leukozytenanzahl > $10 \times 10^{9} / \mathrm{l}$ & 447 & $69,1 \%$ & \\
\hline $\mathrm{CRP}>5 \mathrm{mg} / \mathrm{l}$ & 413 & $64,1 \%$ & \\
\hline
\end{tabular}

Tabelle 6: Apparative Diagnostik im Gesamtkollektiv. Daten als Absolutwerte, prozentualer Anteil der untersuchten Kollektivgröße sowie Mittelwert \pm Standardabweichung.

Tabelle 6 zeigt die Ergebnisse der apparativen Diagnostik. Eine Abdomensonographie war bei etwa $95 \%$ der untersuchten Patienten auswertbar. Auffallend war die Beschreibung eines blanden Sonographiebefundes bei mehr als der Hälfte der appendektomierten Patienten. Eine pathologische Kokarde und freie Flüssigkeit wurden als Einzelbefunde etwa gleich häufig diagnostiziert, traten in Kombination jedoch nur selten auf. Ausgesprochen selten wurde ein perityphlitischer Abszess dokumentiert.

Nur ein geringer Teil des Gesamtkollektivs wurde einer computertomographischen Untersuchung zugeführt. Mit ähnlicher Häufigkeit wurden dabei sowohl eine akute Appendizitis als auch andere radiologische Befunde dokumentiert. 
Innerhalb des Gesamtkollektivs fanden sich weniger Patienten mit Fieber als mit normaler Körpertemperatur.

Die Blutuntersuchung ergab bei etwa zwei Drittel der Patienten einen pathologischen Anstieg der Entzündungsparameter Leukozytenanzahl und CRP. Eine Leukozytose wurde dabei im Vergleich etwas häufiger diagnostiziert (Tabelle 6).

\subsubsection{Perioperative Daten}

Wie in Tabelle 7 dargestellt, vergingen bei mehr als der Hälfte der Patienten maximal 8 Stunden bis zum Operationsbeginn nach stationärer Aufnahme. Bei längerem präoperativem Beobachtungszeitraum verblieben die Patienten am Häufigsten 12 bis 24 Stunden in ärztlicher Überwachung. Die durchschnittliche Operationszeit betrug ungefähr eine Stunde (Tabelle 7).

\begin{tabular}{|l|c|c|c|}
\hline \multicolumn{1}{|c|}{ Parameter } & Absolute Anzahl & Prozentualer Anteil & Mittelwert \pm SD \\
\hline Aufnahme-Schnitt-Dauer $[\mathrm{h}]$ & $\mathbf{6 3 3}$ & & $14,7 \pm 22,2$ \\
$\mathrm{ASD} \leq 4 \mathrm{~h}$ & 176 & $27,8 \%$ & \\
$\mathrm{ASD}>4 \mathrm{bis} \leq 8 \mathrm{~h}$ & 169 & $26,7 \%$ & \\
$\mathrm{ASD}>8$ bis $\leq 12 \mathrm{~h}$ & 55 & $8,7 \%$ & \\
$\mathrm{ASD}>12$ bis $\leq 24 \mathrm{~h}$ & 129 & $20,4 \%$ & \\
$\mathrm{ASD}>24$ bis $\leq 48 \mathrm{~h}$ & 71 & $11,2 \%$ & \\
ASD $>48 \mathrm{~h}$ & 33 & $5,2 \%$ & \\
\hline Operationsdauer $[\mathrm{min}]$ & $\mathbf{6 3 9}$ & & $62,6 \pm 27,2$ \\
\hline
\end{tabular}

Tabelle 7: Perioperatives Zeitmanagement im Gesamtkollektiv. Daten als Absolutwerte, prozentualer Anteil der untersuchten Kollektivgröße sowie Mittelwert \pm Standardabweichung.

Tabelle 8 verdeutlicht eine vermehrte Durchführung von laparoskopischen Appendektomien, wobei die Absetzung der Appendix vermiformis überwiegend mittels Stapler durchgeführt wurde.

Im Falle einer offenen Appendektomie erfolgte bei fast zwei Drittel der Patienten der operative Zugang durch einen Pararektalschnitt (Tabelle 8). 


\begin{tabular}{|l|c|c|}
\hline \multicolumn{1}{|c|}{ Parameter } & Absolute Anzahl & Prozentualer Anteil \\
\hline Operationstechnik & $\mathbf{6 5 5}$ & $37,9 \%$ \\
offen & 248 & $58,5 \%$ \\
laparoskopisch & 383 & $3,7 \%$ \\
konvertiert & 24 & \\
Schnittführung bei OA & $\mathbf{2 1 6}$ & $24,1 \%$ \\
mediane Laparotomie & 52 & $63,9 \%$ \\
Pararektalschnitt & 138 & $12,0 \%$ \\
Wechselschnitt & 26 & $18,3 \%$ \\
\hline Absetzung der Appendix bei LA & $\mathbf{3 8 8}$ & $81,7 \%$ \\
Schlinge & 71 & $39,1 \%$ \\
Stapler & 317 & $14,9 \%$ \\
\hline Drainageneinlage & $\mathbf{2 5 4}^{* 1}$ & $\mathbf{9 7 ^ { * }}$ \\
\hline OP-Erweiterung & & \\
\hline
\end{tabular}

Tabelle 8: Operatives Vorgehen im Gesamtkollektiv. Daten als Absolutwerte und prozentualer Anteil der untersuchten Kollektivgröße ${ }^{* 1}$ Angaben bei 650 Personen; ${ }^{* 2}$ Angaben bei 652 Personen.

\subsubsection{Intraoperativer Befund}

\begin{tabular}{|l|c|c|}
\hline \multicolumn{1}{|c|}{ Parameter } & Absolute Anzahl & Prozentualer Anteil \\
\hline Appendixzustand & $\mathbf{6 4 4}$ & \\
reizlos & 63 & $9,8 \%$ \\
nicht näher definiert akut & 301 & $46,7 \%$ \\
fibrinös & 45 & $7,0 \%$ \\
ulcero-phlegmonös & 153 & $23,8 \%$ \\
abszediert & 10 & $1,6 \%$ \\
gangränös & 12 & $1,9 \%$ \\
perforiert & 103 & $16,0 \%$ \\
Intraoperativer Zusatzbefund & & $18,0 \%$ \\
Peritonitis & $102^{* 3}$ & $33,6 \%$ \\
trübe Flüssigkeit & $189^{* 4}$ & $11,2 \%$ \\
perityphlitischer Abszess & $63^{* 5}$ & \\
\hline
\end{tabular}

Tabelle 9: Intraoperativer Befund des Gesamtkollektivs. Daten als Absolutwerte und prozentualer Anteil der untersuchten Kollektivgröße. ${ }^{* 3}$ Angaben bei 568 Personen; ${ }^{* 4}$ Angaben bei 562 Personen; ${ }^{* 5}$ Angaben bei 563 Personen.

Tabelle 9 fasst die Ergebnisse der chirurgischen Einschätzung des Entzündungszustandes der Appendix vermiformis zusammen. Wie erkennbar, wurde die Appendix bei etwa $10 \%$ der operierten Patienten intraoperativ als reizlos beurteilt. Die restlichen $90 \%$ der operierten Patienten zeigten Entzündungsreaktionen unterschiedlichen Ausmaßes. 
Auffallend häufig wurde keine genaue Definition des Entzündungszustandes der Appendix vermiformis angegeben und die intraoperative Manifestation einer „akuten Appendizitis“ dokumentiert. In beinahe einem Viertel der Operationen notierte der Chirurg das Auffinden einer ulcero-phlegmonösen Appendizitis. Alle anderen Appendizitisstadien wurden deutlich seltener vermerkt.

Trübe Flüssigkeit im Bauchraum stellte den häufigsten intraoperativen Zusatzbefund dar (Tabelle 9).

\subsubsection{Histologischer Befund}

Die Auswertung der histologischen Befunde zeigte das Vorliegen einer reizlosen Appendix vermiformis im Sinne einer negativen Appendektomie in etwa $26 \%$ der Fälle. 74 \% der Präparate waren in unterschiedlicher Ausprägung entzündlich verändert.

Deutlich mehr als die Hälfte der Appendektomiepräparate wurden als akut ulcerophlegmonös entzündet eingestuft. Andere Appendizitisstadien und auch die Beschreibung einer nicht näher definierten akuten Appendizitis waren wesentlich seltener. Auffallend war die außerordentlich seltene histologische Beschreibung einer katarrhalischen oder abszedierten Appendizitis.

Innerhalb des histologischen Zusatzbefundes wurde eine Periappendizitis deutlich häufiger als eine Peritonitis oder Koprostase beschrieben (Tabelle 10).

\begin{tabular}{|l|c|c|}
\hline \multicolumn{1}{|c|}{ Parameter } & Absolute Anzahl & Prozentualer Anteil \\
\hline Appendixzustand & $\mathbf{6 5 4}$ & \\
reizlos & 167 & $25,5 \%$ \\
nicht näher definiert akut & 85 & $13,0 \%$ \\
katarrhalisch & 8 & $1,2 \%$ \\
ulcero-phlegmonös & 382 & $58,4 \%$ \\
abszediert & 8 & $1,2 \%$ \\
gangränös & 49 & $7,5 \%$ \\
perforiert & 62 & $9,5 \%$ \\
\hline Histologischer Zusatzbefund & $\mathbf{6 5 4}$ & $4,7 \%$ \\
Peritonitis & 31 & $41,1 \%$ \\
Periappendizitis & 269 & $15,1 \%$ \\
Koprostase & 99 & \\
\hline
\end{tabular}

Tabelle 10: Histologischer Befund des Gesamtkollektivs. Daten als Absolutwerte und prozentualer Anteil der untersuchten Kollektivgröße. 


\subsubsection{Postoperative Daten}

Wie in Tabelle 11 dargestellt, wurden die Patienten im Mittel etwa 5 Tage nach erfolgter Operation und mit einer Gesamtverweildauer von knapp 6 Tagen entlassen.

Zum überwiegenden Teil verlief die Genesung der Patienten komplikationslos. Wundheilungsstörungen und intraabdominelle Abszesse traten seltener als die unter „Sonstige“ zusammengefassten Probleme auf. Eine operative Revision aufgrund einer postoperativen Komplikation war bei etwa $4 \%$ aller Patienten erforderlich (Tabelle 11).

\begin{tabular}{|l|c|c|c|}
\hline \multicolumn{1}{|c|}{ Parameter } & Absolute Anzahl & Prozentualer Anteil & Mittelwert \pm SD \\
\hline Postoperative Verweildauer [d] & $\mathbf{6 5 5}$ & & $5,2 \pm 7,1$ \\
\hline Gesamtverweildauer [d] & $\mathbf{6 5 4}$ & & $5,9 \pm 8,7$ \\
\hline Postoperativer Verlauf & $\mathbf{6 4 3}$ & $90,7 \%$ & \\
komplikationslos & 583 & $9,3 \%$ & \\
mit Komplikationen & 60 & $3,0 \%$ & \\
\hline Komplikationsarten & 19 & $0,6 \%$ & \\
Wundheilungsstörung & 4 & $7,5 \%$ & \\
intraabdomineller Abszess & 48 & $4,2 \%$ & $1,1 \pm 0,3$ \\
Sonstige & $\mathbf{2 7}$ & $1,8 \%$ & \\
\hline Revisionsoperation [Anzahl] & $\mathbf{1 2 *}$ & & \\
\hline Re-Hospitalisation & & & \\
\hline
\end{tabular}

Tabelle 11: Postoperative Daten des Gesamtkollektivs. Daten als Absolutwerte, prozentualer Anteil der untersuchten Kollektivgröße sowie Mittelwert \pm Standardabweichung. ${ }^{* 6}$ Angaben bei 655 Personen.

\subsection{Subgruppenanalyse}

\subsubsection{Intraoperativer Befund nicht-akute vs. akute Appendizitis}

Tabellen 12 und 13 zeigen die Ergebnisse des Subgruppenvergleichs von Patienten mit dem intraoperativen Befund einer akut entzündeten oder reizlosen Appendix. Die chirurgische Einschätzung des intraoperativen Befundes bezüglich der Appendix war bei 644 Patienten zugänglich. Eine akute Appendizitis wurde hierbei in 581 Fällen und ein reizloser Zustand der Appendix bei 63 Patienten durch den Operateur beschrieben. 


\begin{tabular}{|c|c|c|c|}
\hline & keine akute Appendizitis & akute Appendizitis & $\mathbf{p}$ \\
\hline \multicolumn{4}{|l|}{ Demographische Daten } \\
\hline weibliches Geschlecht & $73,0 \%$ & $50,3 \%$ & 0,001 \\
\hline \multicolumn{4}{|l|}{ Anamnestische Daten } \\
\hline Schmerzen $<4 \mathrm{~h}$ & $7,8 \%$ & $8,1 \%$ & 1,000 \\
\hline Schmerzen 4 bis $<8 \mathrm{~h}$ & $17,6 \%$ & $13,0 \%$ & 0,388 \\
\hline Schmerzen 8 bis $<16 \mathrm{~h}$ & $5,9 \%$ & $19,3 \%$ & 0,020 \\
\hline Schmerzen 16 bis $<24 \mathrm{~h}$ & $5,9 \%$ & $9,7 \%$ & 0,611 \\
\hline Schmerzen $\geq 24 \mathrm{~h}$ & $62,7 \%$ & $49,7 \%$ & 0,080 \\
\hline Kolik & $17,7 \%$ & $11,1 \%$ & 0,143 \\
\hline Dauerschmerz & $82,3 \%$ & $88,0 \%$ & 0,222 \\
\hline Schmerzwanderung & $1,6 \%$ & $22,3 \%$ & $<0,001$ \\
\hline Übelkeit & $37,1 \%$ & $45,8 \%$ & 0,227 \\
\hline Erbrechen & $24,2 \%$ & $32,3 \%$ & 0,249 \\
\hline Diarrhoe & $17,7 \%$ & $18,2 \%$ & 1,000 \\
\hline \multicolumn{4}{|l|}{ Klinische Untersuchung } \\
\hline rechter Unterbauch & $93,5 \%$ & $96,8 \%$ & 0,260 \\
\hline linker Unterbauch & $19,4 \%$ & $21,8 \%$ & 0,747 \\
\hline rechter Oberbauch & $8,1 \%$ & $10,2 \%$ & 0,823 \\
\hline periumbilikal & $14,5 \%$ & $17,0 \%$ & 0,722 \\
\hline epigastrisch & $6,5 \%$ & $9,8 \%$ & 0,498 \\
\hline McBurney-Zeichen & $37,1 \%$ & $31,8 \%$ & 0,395 \\
\hline Lanz-Zeichen & $22,6 \%$ & $21,1 \%$ & 0,746 \\
\hline Loslassschmerz & $27,4 \%$ & $34,1 \%$ & 0,323 \\
\hline Psoasschmerz & $19,4 \%$ & $17,6 \%$ & 0,727 \\
\hline Peritonismus & $6,5 \%$ & $17,0 \%$ & 0,028 \\
\hline Abwehrspannung & $24,2 \%$ & $35,1 \%$ & 0,091 \\
\hline \multicolumn{4}{|l|}{ Apparative Diagnostik } \\
\hline Sono o.p.B. & $80,0 \%$ & $53,7 \%$ & $<0,001$ \\
\hline Sono freie Flüssigkeit & $16,7 \%$ & $17,9 \%$ & 1,000 \\
\hline Sono Kokarde & $1,7 \%$ & $18,4 \%$ & $<0,001$ \\
\hline Sono freie Flüssigkeit und Kokarde & $1,7 \%$ & $7,5 \%$ & 0,109 \\
\hline Sono perityphlitischer Abszess & $0,0 \%$ & $2,6 \%$ & 0,382 \\
\hline CT durchgeführt & $1,6 \%$ & $4,6 \%$ & 0,509 \\
\hline Temperatur $\left[{ }^{\circ} \mathrm{C}\right]$ & $37,1 \pm 1,2$ & $36,9 \pm 0,9$ & 0,095 \\
\hline Temperatur $\leq 37^{\circ} \mathrm{C}$ & $52,4 \%$ & $64,2 \%$ & 0,175 \\
\hline Temperatur 37,1 bis $37,9^{\circ} \mathrm{C}$ & $23,8 \%$ & $20,2 \%$ & 0,551 \\
\hline Temperatur $\geq 38^{\circ} \mathrm{C}$ & $23,8 \%$ & $15,6 \%$ & 0,187 \\
\hline 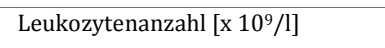 & $10,5 \pm 5,5$ & $13,5 \pm 5,4$ & $<0,001$ \\
\hline CRP $[\mathrm{mg} / \mathrm{l}]$ & $35,8 \pm 62,3$ & $40,5 \pm 61,7$ & 0,573 \\
\hline
\end{tabular}

Tabelle 12: Vergleich anamnestischer, klinischer und apparativ-diagnostischer Befunde von Patienten mit und ohne intraoperativen Befund einer akuten Appendizitis. Daten als prozentualer Anteil oder Mittelwert \pm Standardabweichung, $p$-Wert-Markierung bei $p<0,05$.

Eine negative Appendektomie erfolgte bei signifikant mehr Frauen als Männern, während die Diagnose einer akuten Appendizitis keine Geschlechtsspezifität aufwies.

Kurze Schmerzdauern von unter 8 Stunden waren etwa gleich oft in beiden Untergruppen anzutreffen. Allerdings traten mittlere Beschwerdedauern zwischen 8 bis 16 Stunden signifikant 
häufiger in der Gruppe der akut entzündeten Appendizitiden auf, während Schmerzen seit mehr als 24 Stunden etwas seltener zu finden waren. Das Symptom der Schmerzwanderung zeigte einen hohen Vorhersagewert zugunsten der akut entzündeten Appendix.

Die statistische Auswertung der Subgruppen in Bezug auf die Befunde der körperlichen Untersuchung zeigte kaum signifikante Unterschiede. Erwartungsgemäß wiesen Patienten mit intraoperativem Befund einer akuten Appendizitis jedoch signifikant häufiger das Symptom des Peritonismus auf.

Die sonographische Untersuchung ergab signifikant häufiger einen unauffälligen Befund bei letztlich intraoperativ blander Appendix. Gleichwohl erfolgte der Nachweis eines pathologischen Befundes an weniger als der Hälfte der Patienten mit akuter Appendizitis. Keineswegs spezifisch für das Vorliegen einer akuten Entzündung war die sonographische Darstellung von freier intraabdomineller Flüssigkeit, jedoch manifestierte sich die akut entzündete Appendix mit hoher Signifikanz im sonographischen Nachweis einer pathologischen Kokarde. Bei Patienten mit akuter Appendizitis war im Aufnahmelabor die Leukozytenanzahl im Unterschied zum CRP statistisch signifikant erhöht (Tabelle 12).

\begin{tabular}{|c|c|c|c|}
\hline & keine akute Appendizitis & akute Appendizitis & $\mathbf{p}$ \\
\hline \multicolumn{4}{|l|}{ Perioperatives Zeitmanagement } \\
\hline Aufnahme-Schnitt-Dauer [h] & $22,6 \pm 22,0$ & $13,8 \pm 22,1$ & 0,003 \\
\hline $\mathrm{ASD} \leq 4 \mathrm{~h}$ & $11,1 \%$ & $29,6 \%$ & 0,002 \\
\hline $\mathrm{ASD}>4 \mathrm{bis} \leq 8 \mathrm{~h}$ & $15,9 \%$ & $28,0 \%$ & 0,050 \\
\hline $\mathrm{ASD}>8$ bis $\leq 12 \mathrm{~h}$ & $4,8 \%$ & $9,0 \%$ & 0,345 \\
\hline $\mathrm{ASD}>12$ bis $\leq 24 \mathrm{~h}$ & $36,5 \%$ & $18,5 \%$ & 0,002 \\
\hline ASD $>24$ bis $\leq 48 \mathrm{~h}$ & $22,2 \%$ & $10,1 \%$ & 0,010 \\
\hline $\mathrm{ASD}>48 \mathrm{~h}$ & $9,5 \%$ & $4,8 \%$ & 0,128 \\
\hline Operationsdauer [min] & $69,6 \pm 32,2$ & $61,9 \pm 26,4$ & 0,031 \\
\hline \multicolumn{4}{|l|}{ Operatives Vorgehen } \\
\hline offen & $17,5 \%$ & $39,8 \%$ & $<0,001$ \\
\hline laparoskopisch & $79,4 \%$ & $56,5 \%$ & $<0,001$ \\
\hline konvertiert & $3,2 \%$ & $3,8 \%$ & 1,000 \\
\hline \multicolumn{4}{|l|}{ Postoperative Daten } \\
\hline postoperative Verweildauer [d] & $4,8 \pm 4,3$ & $5,3 \pm 7,4$ & 0,606 \\
\hline Gesamtverweildauer [d] & $5,8 \pm 4,6$ & $6,0 \pm 9,1$ & 0,872 \\
\hline komplikationslos & $90,2 \%$ & $90,9 \%$ & 0,815 \\
\hline Wundheilungsstörung & $0,0 \%$ & $3,3 \%$ & 0,242 \\
\hline intraabdomineller Abszess & $0,0 \%$ & $0,7 \%$ & 1,000 \\
\hline Revisionsoperation & $6,6 \%$ & $3,8 \%$ & 0,302 \\
\hline Sonstige & $9,8 \%$ & $7,0 \%$ & 0,432 \\
\hline Re-Hospitalisation & $4,8 \%$ & $1,5 \%$ & 0,104 \\
\hline
\end{tabular}

Tabelle 13: Vergleich peri- und postoperativer Parameter von Patienten mit und ohne intraoperativen Befund einer akuten Appendizitis. Daten als prozentualer Anteil oder Mittelwert \pm Standardabweichung, p-Wert-Markierung bei $p<0,05$. 
Die Dauer von Patientenvorstellung bis Operationsbeginn war bei Patienten mit akuter Appendizitis signifikant kürzer als bei Patienten ohne akut entzündlichen Befund. Auch die Operationszeit zeigte sich im Falle einer akuten Appendizitis signifikant verkürzt.

Eine Entscheidung des Chirurgen zugunsten eines offenen Zuganges wurde bei akuter Appendizitis deutlich häufiger getroffen.

Patienten, bei denen der intraoperative Nachweis einer akuten Appendizitis erfolgte, wiesen keine signifikant längere stationäre Verweildauer im Vergleich zu Patienten ohne akute Appendizitis auf. Bemerkenswert war die gleiche Komplikationsrate zwischen Patienten mit und ohne akute Appendizitis, wobei sich die Art der Komplikationen unterschied. Während in der Subgruppe der Patienten mit akuter Appendizitis Wundheilungsstörungen, intraabdominelle Abszesse und Revisionsoperationen neben sonstigen postoperativen Problemen auftraten, dominierten bei Patienten mit intraoperativ blander Appendix die Komplikationsarten Revisionsoperationen, sonstige Probleme und Re-Hospitalisationen. Eine statistische Signifikanz war hierbei jedoch nicht nachweisbar (Tabelle 13).

\subsubsection{Histologischer Befund nicht-akute vs. akute Appendizitis}

In den Tabellen 14 und 15 werden die Ergebnisse des Subgruppenvergleichs von Patienten mit dem histologischen Nachweis einer akut entzündeten oder blanden Appendix präsentiert. Ein histologischer Befund der Operationspräparate konnte bei 654 Patienten ermittelt werden, 487 Patienten mit der histologischen Diagnose einer akuten Appendizitis und 167 Patienten mit blander Appendix vermiformis.

Es war eine Geschlechtsspezifität der negativen Appendektomierate erkennbar. Signifikant mehr weibliche Patienten mit letztlich histologisch blandem Befund wurden appendektomiert. Jedoch wurde eine akute Appendizitis mit gleicher Häufigkeit an Männern und Frauen diagnostiziert.

Eine mittlere Schmerzdauer von 8 bis 16 Stunden war vorherrschend bei Patienten mit akuter Appendizitis. Beschwerden seit mehr als einem Tag kamen prozentual seltener in dieser Gruppe vor. Auch Schmerzwanderung, Übelkeit und Erbrechen waren hochsignifikant mit der späteren histologischen Diagnose einer akuten Appendizitis vergesellschaftet.

Bei histologisch akut entzündeter Appendix zeigte sich im Rahmen der klinischen Untersuchung deutlich häufiger epigastrischer Druckschmerz. Alle anderen Palpationspunkte wiesen keinen signifikanten Häufigkeitsunterschied auf. Unter den typischen Appendizitiszeichen war lediglich Peritonismus deutlich öfter bei Patienten mit akuter Entzündung zu finden.

Die abdominelle Ultraschalluntersuchung ergab erwartungsgemäß signifikant seltener einen unauffälligen Befund bei histologisch akuter Appendizitis. Trotzdem konnte bei mehr als der Hälfte der Patienten mit akuter Entzündung keine sonographische Darstellung eines pathologi- 
schen Zustandes erfolgen. Der Anteil der Darstellungen pathologischer Kokarden und perityphlitischer Abszesse war bei Patienten mit akuter Appendizitis signifikant erhöht. Des Weiteren waren in dieser Patientengruppe die Leukozytenanzahl und der CRP-Wert deutlich höher als bei Patienten ohne histologischen Befund einer akuten Appendizitis (Tabelle 14).

\begin{tabular}{|c|c|c|c|}
\hline & keine akute Appendizitis & akute Appendizitis & $\mathbf{p}$ \\
\hline \multicolumn{4}{|l|}{ Demographische Daten } \\
\hline weibliches Geschlecht & $62,3 \%$ & $49,1 \%$ & 0,004 \\
\hline \multicolumn{4}{|l|}{ Anamnestische Daten } \\
\hline Schmerzen $<4 \mathrm{~h}$ & $9,3 \%$ & $8,0 \%$ & 0,610 \\
\hline Schmerzen 4 bis $<8 \mathrm{~h}$ & $9,9 \%$ & $14,5 \%$ & 0,167 \\
\hline Schmerzen 8 bis $<16 \mathrm{~h}$ & $10,6 \%$ & $20,0 \%$ & 0,009 \\
\hline Schmerzen 16 bis $<24 \mathrm{~h}$ & $5,3 \%$ & $10,7 \%$ & 0,052 \\
\hline Schmerzen $\geq 24 \mathrm{~h}$ & $64,9 \%$ & $46,6 \%$ & $<0,001$ \\
\hline Kolik & $12,7 \%$ & $11,1 \%$ & 0,574 \\
\hline Dauerschmerz & $86,7 \%$ & $88,0 \%$ & 0,680 \\
\hline Schmerzwanderung & $10,9 \%$ & $23,5 \%$ & $<0,001$ \\
\hline Übelkeit & $29,1 \%$ & $50,5 \%$ & $<0,001$ \\
\hline Erbrechen & $17,6 \%$ & $36,3 \%$ & $<0,001$ \\
\hline Diarrhoe & $13,3 \%$ & $19,7 \%$ & 0,078 \\
\hline \multicolumn{4}{|l|}{ Klinische Untersuchung } \\
\hline rechter Unterbauch & $96,3 \%$ & $96,6 \%$ & 0,808 \\
\hline linker Unterbauch & $21,3 \%$ & $21,5 \%$ & 1,000 \\
\hline rechter Oberbauch & $8,5 \%$ & $10,3 \%$ & 0,649 \\
\hline periumbilikal & $16,5 \%$ & $16,6 \%$ & 1,000 \\
\hline epigastrisch & $5,5 \%$ & $10,9 \%$ & 0,045 \\
\hline McBurney-Zeichen & $34,1 \%$ & $32,0 \%$ & 0,630 \\
\hline Lanz-Zeichen & $18,3 \%$ & $22,3 \%$ & 0,320 \\
\hline Loslassschmerz & $28,7 \%$ & $35,6 \%$ & 0,125 \\
\hline Psoasschmerz & $21,3 \%$ & $16,2 \%$ & 0,153 \\
\hline Peritonismus & $9,8 \%$ & $17,9 \%$ & 0,013 \\
\hline Abwehrspannung & $34,1 \%$ & $34,3 \%$ & 1,000 \\
\hline \multicolumn{4}{|l|}{ Apparative Diagnostik } \\
\hline Sono o.p.B. & $72,8 \%$ & $51,0 \%$ & $<0,001$ \\
\hline Sono freie Flüssigkeit & $17,1 \%$ & $17,8 \%$ & 0,904 \\
\hline Sono Kokarde & $4,4 \%$ & $21,0 \%$ & $<0,001$ \\
\hline Sono freie Flüssigkeit und Kokarde & $5,7 \%$ & $7,2 \%$ & 0,588 \\
\hline Sono perityphlitischer Abszess & $0,0 \%$ & $3,0 \%$ & 0,026 \\
\hline CT durchgeführt & $1,8 \%$ & $5,1 \%$ & 0,076 \\
\hline Temperatur $\left[{ }^{\circ} \mathrm{C}\right]$ & $36,9 \pm 1,0$ & $36,9 \pm 0,9$ & 0,674 \\
\hline Temperatur $\leq 37^{\circ} \mathrm{C}$ & $70,3 \%$ & $60,7 \%$ & 0,095 \\
\hline Temperatur 37,1 bis $37,9^{\circ} \mathrm{C}$ & $14,9 \%$ & $22,4 \%$ & 0,117 \\
\hline Temperatur $\geq 38^{\circ} \mathrm{C}$ & $14,9 \%$ & $16,9 \%$ & 0,756 \\
\hline Leukozytenanzahl $\left[\mathrm{x} 10^{9} / \mathrm{l}\right]$ & $10,7 \pm 5,3$ & $14,0 \pm 5,3$ & $<0,001$ \\
\hline $\mathrm{CRP}[\mathrm{mg} / \mathrm{l}]$ & $31,1 \pm 52,3$ & $43,6 \pm 65,1$ & 0,014 \\
\hline
\end{tabular}

Tabelle 14: Vergleich anamnestischer, klinischer und apparativ-diagnostischer Befunde von Patienten mit und ohne histologischen Befund einer akuten Appendizitis. Daten als prozentualer Anteil oder Mittelwert \pm Standardabweichung, $p$-Wert-Markierung bei $p<0,05$. 
Die Analyse der Aufnahme-Schnitt-Dauer zeigte eine schnellere Versorgung der Patienten mit akuter Appendizitis, wobei insbesondere im Zeitintervall von bis zu 4 Stunden signifikant mehr Patienten einer Operation zugeführt wurden. Hingegen wurden Patienten mit histologisch nachgewiesener akuter Appendizitis signifikant seltener später als 12 Stunden nach stationärer Aufnahme operiert.

Bei Patienten mit histologisch akutem Befund wurde signifikant häufiger ein offenes chirurgisches Vorgehen angewandt.

Der postoperative stationäre Aufenthalt sowie die Gesamtverweildauer waren bei Patienten mit histologisch akuter Appendizitis nicht deutlich länger als bei Patienten ohne akute Appendizitis. Die postoperative Komplikationsrate zeigte sich in beiden Subgruppen nahezu identisch (Tabelle 15).

\begin{tabular}{|c|c|c|c|}
\hline & keine akute Appendizitis & akute Appendizitis & $\mathbf{p}$ \\
\hline \multicolumn{4}{|l|}{ Perioperatives Zeitmanagement } \\
\hline Aufnahme-Schnitt-Dauer [h] & $16,9 \pm 17,9$ & $13,9 \pm 23,5$ & 0,140 \\
\hline $\mathrm{ASD} \leq 4 \mathrm{~h}$ & $20,2 \%$ & $30,5 \%$ & 0,011 \\
\hline $\mathrm{ASD}>4 \mathrm{bis} \leq 8 \mathrm{~h}$ & $21,5 \%$ & $28,4 \%$ & 0,100 \\
\hline $\mathrm{ASD}>8$ bis $\leq 12 \mathrm{~h}$ & $8,6 \%$ & $8,7 \%$ & 1,000 \\
\hline ASD $>12$ bis $\leq 24 \mathrm{~h}$ & $27,6 \%$ & $17,9 \%$ & 0,010 \\
\hline $\mathrm{ASD}>24$ bis $\leq 48 \mathrm{~h}$ & $16,0 \%$ & $9,6 \%$ & 0,031 \\
\hline $\mathrm{ASD}>48 \mathrm{~h}$ & $6,1 \%$ & $4,9 \%$ & 0,543 \\
\hline Operationsdauer [min] & $63,7 \pm 34,0$ & $62,2 \pm 24,5$ & 0,609 \\
\hline \multicolumn{4}{|l|}{ Operatives Vorgehen } \\
\hline offen & $28,1 \%$ & $41,3 \%$ & 0,003 \\
\hline laparoskopisch & $68,9 \%$ & $54,8 \%$ & 0,001 \\
\hline konvertiert & $3,0 \%$ & $3,9 \%$ & 0,812 \\
\hline \multicolumn{4}{|l|}{ Postoperative Daten } \\
\hline postoperative Verweildauer [d] & $4,7 \pm 5,0$ & $5,4 \pm 7,7$ & 0,262 \\
\hline Gesamtverweildauer [d] & $5,4 \pm 5,1$ & $6,1 \pm 9,7$ & 0,327 \\
\hline komplikationslos & $90,9 \%$ & $90,6 \%$ & 1,000 \\
\hline Wundheilungsstörung & $2,4 \%$ & $3,1 \%$ & 0,793 \\
\hline intraabdomineller Abszess & $0,6 \%$ & $0,6 \%$ & 1,000 \\
\hline Revisionsoperation & $4,2 \%$ & $4,2 \%$ & 1,000 \\
\hline Sonstige & $7,9 \%$ & $7,3 \%$ & 0,864 \\
\hline Re-Hospitalisation & $1,8 \%$ & $1,8 \%$ & 1,000 \\
\hline
\end{tabular}

Tabelle 15: Vergleich peri- und postoperativer Parameter von Patienten mit und ohne histologischen Befund einer akuten Appendizitis. Daten als prozentualer Anteil oder Mittelwert \pm Standardabweichung, $p$-Wert-Markierung bei $p<0,05$.

\subsubsection{Nicht-komplizierter vs. komplizierter postoperativer Verlauf}

Tabellen 16 und 17 präsentieren die Ergebnisse des Vergleichs von Patienten mit postoperativ kompliziertem und komplikationslosem Verlauf. Bei 643 Patienten konnte eine Datenerhebung des postoperativen stationären Aufenthalts bei vorhandenen Daten erfolgen. Von diesen 643 
Patienten zeigten 60 Patienten Komplikationen, während bei 583 Personen ein komplikationsfreier Genesungsverlauf dokumentiert wurde.

\begin{tabular}{|c|c|c|c|}
\hline & komplikationslos & Komplikationen & $\mathbf{p}$ \\
\hline \multicolumn{4}{|l|}{ Demographische Daten } \\
\hline weibliches Geschlecht & $53,5 \%$ & $40,0 \%$ & 0,057 \\
\hline Alter [y] & $27,7 \pm 18,2$ & $35,1 \pm 26,5$ & 0,038 \\
\hline \multicolumn{4}{|l|}{ Anamnestische Daten } \\
\hline Schmerzen $<4 \mathrm{~h}$ & $8,2 \%$ & $6,8 \%$ & 0,788 \\
\hline Schmerzen 4 bis $<8 \mathrm{~h}$ & $13,3 \%$ & $13,6 \%$ & 1,000 \\
\hline Schmerzen 8 bis $<16 \mathrm{~h}$ & $18,0 \%$ & $15,7 \%$ & 0,849 \\
\hline Schmerzen 16 bis $<24 \mathrm{~h}$ & $9,4 \%$ & $9,8 \%$ & 1,000 \\
\hline Schmerzen $\geq 24 \mathrm{~h}$ & $50,9 \%$ & $53,9 \%$ & 0,772 \\
\hline Kolik & $11,8 \%$ & $7,0 \%$ & 0,382 \\
\hline Dauerschmerz & $87,7 \%$ & $89,5 \%$ & 0,833 \\
\hline Schmerzwanderung & $21,0 \%$ & $15,8 \%$ & 0,396 \\
\hline Übelkeit & $45,2 \%$ & $44,8 \%$ & 1,000 \\
\hline Erbrechen & $31,5 \%$ & $31,0 \%$ & 1,000 \\
\hline Diarrhoe & $17,3 \%$ & $25,9 \%$ & 0,110 \\
\hline \multicolumn{4}{|l|}{ Klinische Untersuchung } \\
\hline rechter Unterbauch & $96,9 \%$ & $92,7 \%$ & 0,115 \\
\hline linker Unterbauch & $21,1 \%$ & $25,5 \%$ & 0,492 \\
\hline rechter Oberbauch & $8,7 \%$ & $21,8 \%$ & 0,007 \\
\hline periumbilikal & $15,7 \%$ & $23,6 \%$ & 0,131 \\
\hline epigastrisch & $8,8 \%$ & $16,4 \%$ & 0,087 \\
\hline McBurney-Zeichen & $34,1 \%$ & $18,2 \%$ & 0,016 \\
\hline Lanz-Zeichen & $22,0 \%$ & $16,4 \%$ & 0,393 \\
\hline Loslassschmerz & $34,3 \%$ & $27,3 \%$ & 0,370 \\
\hline Psoasschmerz & $18,2 \%$ & $10,9 \%$ & 0,199 \\
\hline Peritonismus & $16,1 \%$ & $12,7 \%$ & 0,698 \\
\hline Abwehrspannung & $32,7 \%$ & $45,5 \%$ & 0,073 \\
\hline \multicolumn{4}{|l|}{ Apparative Diagnostik } \\
\hline Sono o.p.B. & $57,2 \%$ & $50,0 \%$ & 0,324 \\
\hline Sono freie Flüssigkeit & $16,4 \%$ & $26,8 \%$ & 0,063 \\
\hline Sono Kokarde & $17,4 \%$ & $10,7 \%$ & 0,261 \\
\hline Sono freie Flüssigkeit und Kokarde & $7,2 \%$ & $5,4 \%$ & 0,787 \\
\hline Sono perityphlitischer Abszess & $1,8 \%$ & $7,1 \%$ & 0,031 \\
\hline CT durchgeführt & $3,6 \%$ & $10,0 \%$ & 0,032 \\
\hline Temperatur $\left[{ }^{\circ} \mathrm{C}\right]$ & $36,9 \pm 0,9$ & $37,9 \pm 1,1$ & 0,004 \\
\hline Temperatur $\leq 37^{\circ} \mathrm{C}$ & $64,6 \%$ & $44,1 \%$ & 0,025 \\
\hline Temperatur 37,1 bis $37,9^{\circ} \mathrm{C}$ & $19,8 \%$ & $29,4 \%$ & 0,187 \\
\hline Temperatur $\geq 38^{\circ} \mathrm{C}$ & $15,6 \%$ & $26,5 \%$ & 0,143 \\
\hline Leukozytenanzahl [x 109/l] & $13,0 \pm 5,3$ & $14,6 \pm 6,6$ & 0,032 \\
\hline CRP $[\mathrm{mg} / \mathrm{l}]$ & $36,8 \pm 56,1$ & $82,1 \pm 96,1$ & $<0,001$ \\
\hline
\end{tabular}

Tabelle 16: Vergleich anamnestischer, klinischer und apparativ-diagnostischer Befunde von Patienten mit komplikationsbehaftetem und komplikationslosem postoperativem Verlauf. Daten als prozentualer Anteil oder Mittelwert \pm Standardabweichung, $p$-Wert-Markierung bei $p<0,05$. 
Patienten mit Komplikationen waren häufiger männlichen Geschlechts und darüber hinaus signifikant älter.

Der Vergleich der anamnestisch erhobenen Parameter zeigte keine statistisch signifikanten Unterschiede zwischen Patienten mit komplikationsbehaftetem und komplikationslosem postoperativem Verlauf.

Bei der klinischen Untersuchung war das Symptom des rechtsseitigen Oberbauchschmerzes bei Patienten mit nachfolgend komplikativem Verlauf signifikant häufiger nachzuweisen. Alle typischen Appendizitiszeichen - mit Ausnahme des Symptoms der Abwehrspannung - wurden seltener bei Patienten mit komplikationsbehaftetem postoperativem Intervall nachgewiesen.

Bei postoperativ komplikationsbehaftetem Aufenthalt gelang im Voraus signifikant häufiger der sonographische Nachweis eines perityphlitischen Abszesses. Des Weiteren hatten Patienten mit postoperativ kompliziertem Krankheitsverlauf höhere systemische Infektparameter und bedurften deutlich häufiger der Durchführung einer CT-Untersuchung im Rahmen der präoperativen Diagnostik (Tabelle 16).

\begin{tabular}{|c|c|c|c|}
\hline & komplikationslos & Komplikationen & $\mathbf{p}$ \\
\hline \multicolumn{4}{|c|}{ Perioperatives Zeitmanagement } \\
\hline Aufnahme-Schnitt-Dauer [h] & $14,3 \pm 22,0$ & $16,7 \pm 22,3$ & 0,437 \\
\hline $\mathrm{ASD} \leq 4 \mathrm{~h}$ & $27,9 \%$ & $29,8 \%$ & 0,758 \\
\hline $\mathrm{ASD}>4 \mathrm{bis} \leq 8 \mathrm{~h}$ & $26,5 \%$ & $28,1 \%$ & 0,756 \\
\hline $\mathrm{ASD}>8$ bis $\leq 12 \mathrm{~h}$ & $9,2 \%$ & $5,3 \%$ & 0,462 \\
\hline ASD $>12$ bis $\leq 24 \mathrm{~h}$ & $21,0 \%$ & $12,3 \%$ & 0,164 \\
\hline $\mathrm{ASD}>24$ bis $\leq 48 \mathrm{~h}$ & $10,9 \%$ & $15,8 \%$ & 0,274 \\
\hline $\mathrm{ASD}>48 \mathrm{~h}$ & $4,6 \%$ & $8,8 \%$ & 0,191 \\
\hline Operationsdauer [min] & $62,0 \pm 26,2$ & $69,1 \pm 34,0$ & 0,104 \\
\hline \multicolumn{4}{|l|}{ Operatives Vorgehen } \\
\hline offen & $35,7 \%$ & $60,0 \%$ & $<0,001$ \\
\hline laparoskopisch & $61,2 \%$ & $33,3 \%$ & $<0,001$ \\
\hline konvertiert & $3,1 \%$ & $6,7 \%$ & 1,000 \\
\hline
\end{tabular}

Tabelle 17: Vergleich peri- und postoperativer Parameter von Patienten mit komplikationsbehaftetem und komplikationslosem postoperativem Verlauf. Daten als prozentualer Anteil oder Mittelwert \pm Standardabweichung, $p$-WertMarkierung bei $p<0,05$.

Patienten mit komplikationsbehaftetem Verlauf hatten im Mittel eine etwas längere Aufnahme-Schnitt-Dauer, wobei sie seltener als Patienten ohne postoperative Komplikationen im Zeitraum von 8 bis 24 Stunden einer Operation zugeführt wurden. Eine statistische Signifikanz konnte hierbei allerdings nicht nachgewiesen werden.

Das Auftreten postoperativer Probleme war von der Operationsdauer unabhängig, wobei Patienten mit postoperativen Komplikationen signifikant häufiger offen appendektomiert wurden (Tabelle 17). 
In der Patientengruppe mit komplikationsbehaftetem Verlauf waren intraoperative Manifestationen einer fortgeschrittenen Appendizitis wie Perforation, Peritonitis und Abszessbildung signifikant häufiger nachweisbar $(\mathrm{p}<0,001)$. Ebenso ergab die zugehörige histologische Befundung bei Patienten mit komplikativem Verlauf deutlich häufiger fortgeschrittene Entzündungsstadien wie eine gangränöse $(p=0,005)$ oder perforierte Appendizitis $(p=0,001)$ im Vergleich $z u$ Patienten mit komplikationslosem postoperativem Verlauf.

\subsubsection{Nicht-perforierte vs. perforierte Appendizitis}

Die Tabellen 18 und 19 zeigen die Ergebnisse des Subgruppenvergleichs von Patienten mit und ohne intraoperativ diagnostizierte Perforation der Appendix. Eine Aussage zum intraoperativen Zustand der Appendix vermiformis konnte bei insgesamt 644 Personen ermittelt werden, 103 Patienten mit der intraoperativen Diagnose einer perforierten Appendizitis und 541 Patienten ohne intraoperativ diagnostizierte Appendixperforation.

Männliche Patienten wiesen signifikant häufiger den intraoperativen Befund einer perforierten Appendizitis auf. Patienten mit einer perforierten Appendizitis waren signifikant älter als Patienten mit nicht-perforierter Appendizitis.

Die Analyse der Schmerzdauer vor stationärer Aufnahme zeigte, dass Patienten mit perforierter Appendizitis signifikant häufiger eine Beschwerdedauer von mehr als 24 Stunden äußerten, wohingegen eine kurze Schmerzanamnese von 4 bis 8 Stunden bei Patienten ohne Appendixperforation deutlich häufiger erhoben wurde. Patienten mit perforierter Appendizitis litten signifikant häufiger an den Symptomen Übelkeit, Erbrechen und Schmerzwanderung. Die Schmerzcharakteristika Kolik und Dauerschmerz traten dagegen in beiden Subgruppen mit gleicher Häufigkeit auf.

Die Analyse der klinischen Untersuchungsbefunde zeigte bei Patienten mit perforierter Appendizitis signifikant häufiger Druckschmerz im linken Unter- und rechten Oberbauch, epigastrisch sowie Peritonismus und Abwehrspannung.

In der Gruppe der Patienten mit Appendixperforation wurden einerseits sonographisch signifikant häufiger freie Flüssigkeit und perityphlitische Abszesse, andererseits signifikant seltener unauffällige sonographische Befunde erhoben. Die Durchführung einer CT-Untersuchung im Rahmen der präoperativen Diagnostik war deutlich häufiger bei Patienten mit perforierter Appendizitis erforderlich. Die Patienten mit perforierter Appendizitis zeigten signifikant ausgeprägtere Zeichen einer systemischen Entzündungsreaktion wie erhöhte Körpertemperatur, Leukozytenzahlen und CRP-Werte (Tabelle 18). 


\begin{tabular}{|c|c|c|c|}
\hline & keine Perforation & Perforation & $\mathbf{p}$ \\
\hline \multicolumn{4}{|l|}{ Demographische Daten } \\
\hline weibliches Geschlecht & $55,1 \%$ & $41,0 \%$ & 0,008 \\
\hline Alter $[y]$ & $26,4 \pm 17,1$ & $37,6 \pm 24,6$ & $<0,001$ \\
\hline \multicolumn{4}{|l|}{ Anamnestische Daten } \\
\hline Schmerzen $<4 \mathrm{~h}$ & $8,8 \%$ & $4,8 \%$ & 0,234 \\
\hline Schmerzen 4 bis $<8 \mathrm{~h}$ & $15,8 \%$ & $2,9 \%$ & $<0,001$ \\
\hline Schmerzen 8 bis $<16 \mathrm{~h}$ & $18,9 \%$ & $13,3 \%$ & 0,206 \\
\hline Schmerzen 16 bis $<24 \mathrm{~h}$ & $9,5 \%$ & $9,5 \%$ & 1,000 \\
\hline Schmerzen $\geq 24 \mathrm{~h}$ & $46,7 \%$ & $69,5 \%$ & $<0,001$ \\
\hline Kolik & $11,8 \%$ & $11,7 \%$ & 1,000 \\
\hline Dauerschmerz & $87,5 \%$ & $87,4 \%$ & 1,000 \\
\hline Schmerzwanderung & $18,5 \%$ & $27,9 \%$ & 0,027 \\
\hline Übelkeit & $42,6 \%$ & $56,3 \%$ & 0,009 \\
\hline Erbrechen & $27,6 \%$ & $50,0 \%$ & $<0,001$ \\
\hline Diarrhoe & $17,0 \%$ & $24,1 \%$ & 0,080 \\
\hline \multicolumn{4}{|l|}{ Klinische Untersuchung } \\
\hline rechter Unterbauch & $96,5 \%$ & $96,4 \%$ & 1,000 \\
\hline linker Unterbauch & $18,0 \%$ & $38,4 \%$ & $<0,001$ \\
\hline rechter Oberbauch & $8,7 \%$ & $16,1 \%$ & 0,024 \\
\hline periumbilikal & $15,8 \%$ & $21,4 \%$ & 0,164 \\
\hline epigastrisch & $8,1 \%$ & $16,1 \%$ & 0,013 \\
\hline McBurney-Zeichen & $33,8 \%$ & $25,9 \%$ & 0,119 \\
\hline Lanz-Zeichen & $21,2 \%$ & $21,4 \%$ & 1,000 \\
\hline Loslassschmerz & $33,4 \%$ & $33,0 \%$ & 1,000 \\
\hline Psoasschmerz & $18,7 \%$ & $13,4 \%$ & 0,220 \\
\hline Peritonismus & $14,3 \%$ & $24,1 \%$ & 0,015 \\
\hline Abwehrspannung & $31,7 \%$ & $45,5 \%$ & 0,006 \\
\hline \multicolumn{4}{|l|}{ Apparative Diagnostik } \\
\hline Sono o.p.B. & $60,6 \%$ & $37,3 \%$ & $<0,001$ \\
\hline Sono freie Flüssigkeit & $15,9 \%$ & $26,4 \%$ & 0,013 \\
\hline Sono Kokarde & $16,5 \%$ & $18,2 \%$ & 0,673 \\
\hline Sono freie Flüssigkeit und Kokarde & $6,6 \%$ & $7,3 \%$ & 0,834 \\
\hline Sono perityphlitischer Abszess & $0,4 \%$ & $10,9 \%$ & $<0,001$ \\
\hline CT durchgeführt & $2,3 \%$ & $13,7 \%$ & $<0,001$ \\
\hline Temperatur $\left[{ }^{\circ} \mathrm{C}\right]$ & $36,9 \pm 0,9$ & $37,2 \pm 1,0$ & 0,012 \\
\hline Temperatur $\leq 37^{\circ} \mathrm{C}$ & $65,7 \%$ & $48,4 \%$ & 0,011 \\
\hline Temperatur 37,1 bis $37,9^{\circ} \mathrm{C}$ & $21,0 \%$ & $18,8 \%$ & 0,739 \\
\hline Temperatur $\geq 38^{\circ} \mathrm{C}$ & $13,3 \%$ & $32,8 \%$ & $<0,001$ \\
\hline Leukozytenanzahl [x 109/l] & $12,6 \pm 5,0$ & $16,0 \pm 6,6$ & $<0,001$ \\
\hline $\mathrm{CRP}[\mathrm{mg} / \mathrm{l}]$ & $29,6 \pm 50,1$ & $86,7 \pm 83,7$ & $<0,001$ \\
\hline
\end{tabular}

Tabelle 18: Vergleich anamnestischer, klinischer und apparativ-diagnostischer Befunde von Patienten mit perforierter und nicht-perforierter Appendizitis. Daten als prozentualer Anteil oder Mittelwert \pm Standardabweichung, $p$-WertMarkierung bei $p<0,05$.

Der Vergleich der Aufnahme-Schnitt-Dauer offenbarte, dass Patienten mit perforierter Appendizitis 7 Stunden länger bis zum Operationsbeginn hospitalisiert waren, wenngleich dieser Unterschied nur einen Trend zeigte und nicht statistisch signifikant war. Als Ausdruck des kom- 
plizierten intraoperativen Befundes war die Operationszeit in dieser Subgruppe signifikant länger. Patienten mit Perforation wurden signifikant häufiger primär offen operiert und erfuhren häufiger eine Konversion bei laparoskopischem Operationsbeginn.

Die Analyse der postoperativen Parameter zeigte bei Patienten mit Appendixperforation eine signifikant längere stationäre Verweildauer sowie eine wesentlich höhere Komplikationsrate. Insbesondere Wundheilungsstörungen, Revisionsoperationen und sonstige Komplikationen traten häufiger im Falle einer perforierten Appendizitis auf (Tabelle 19).

\begin{tabular}{|c|c|c|c|}
\hline & keine Perforation & Perforation & $\mathbf{p}$ \\
\hline \multicolumn{4}{|l|}{ Perioperatives Zeitmanagement } \\
\hline Aufnahme-Schnitt-Dauer [h] & $13,5 \pm 15,7$ & $20,5 \pm 40,3$ & 0,070 \\
\hline $\mathrm{ASD} \leq 4 \mathrm{~h}$ & $27,3 \%$ & $30,4 \%$ & 0,561 \\
\hline $\mathrm{ASD}>4$ bis $\leq 8 \mathrm{~h}$ & $26,5 \%$ & $27,7 \%$ & 0,814 \\
\hline $\mathrm{ASD}>8$ bis $\leq 12 \mathrm{~h}$ & $8,9 \%$ & $7,1 \%$ & 0,710 \\
\hline ASD $>12$ bis $\leq 24 \mathrm{~h}$ & $21,7 \%$ & $14,3 \%$ & 0,092 \\
\hline ASD $>24$ bis $\leq 48 \mathrm{~h}$ & $11,6 \%$ & $9,8 \%$ & 0,742 \\
\hline$A S D>48 h$ & $4,1 \%$ & $10,7 \%$ & 0,008 \\
\hline Operationsdauer [min] & $60,0 \pm 25,1$ & $75,2 \pm 34,0$ & $<0,001$ \\
\hline \multicolumn{4}{|l|}{ Operatives Vorgehen } \\
\hline offen & $34,0 \%$ & $53,8 \%$ & $<0,001$ \\
\hline laparoskopisch & $63,7 \%$ & $35,9 \%$ & $<0,001$ \\
\hline konvertiert & $2,3 \%$ & $10,3 \%$ & $<0,001$ \\
\hline \multicolumn{4}{|l|}{ Postoperative Daten } \\
\hline postoperative Verweildauer [d] & $4,2 \pm 5,1$ & $9,6 \pm 12,0$ & $<0,001$ \\
\hline Gesamtverweildauer [d] & $4,8 \pm 5,1$ & $11,2 \pm 16,6$ & $<0,001$ \\
\hline komplikationslos & $93,1 \%$ & $80,4 \%$ & $<0,001$ \\
\hline Wundheilungsstörung & $1,9 \%$ & $8,0 \%$ & 0,002 \\
\hline intraabdomineller Abszess & $0,4 \%$ & $1,8 \%$ & 0,146 \\
\hline Revisionsoperation & $3,3 \%$ & $8,0 \%$ & 0,032 \\
\hline Sonstige & $5,6 \%$ & $15,2 \%$ & 0,001 \\
\hline Re-Hospitalisation & $1,7 \%$ & $2,6 \%$ & 0,465 \\
\hline
\end{tabular}

Tabelle 19: Vergleich peri- und postoperativer Parameter von Patienten mit perforierter und nicht-perforierter Appendizitis. Daten als prozentualer Anteil oder Mittelwert \pm Standardabweichung, $p$-Wert-Markierung bei $p<0,05$.

Zur Beantwortung der Frage, ob die Perforation der Appendix vermiformis als fortgeschrittenes Entzündungsstadium der Appendizitis bereits bei Vorstellung des Patienten besteht oder erst durch verzögerte chirurgische Diagnose- und Indikationsstellung auftritt, dient Abbildung 2. Es wurde das kumulative relative Auftreten einer perforierten Appendizitis gegen die Dauer von Aufnahme bis Operationsbeginn aufgetragen.

Obwohl etwa $80 \%$ der Personen in der Patientengruppe mit perforierter Appendizitis innerhalb von 24 Stunden nach Aufnahme operiert wurden, musste intraoperativ ein deutlich fortgeschrittener Entzündungsbefund festgestellt werden. Etwa $58 \%$ der Patienten mit perforierter Appendizitis wurden nach maximal 8 Stunden operiert. Die Analyse zeigt, dass kumulati- 
ve $95 \%$ aller Patienten mit dem Nachweis einer Perforation bis zu einem Zeitpunkt von 94 Stunden nach Aufnahme operiert wurden, was Ausdruck der mitunter späten klinischen Manifestation einer Appendixperforation ist (Abbildung 2).

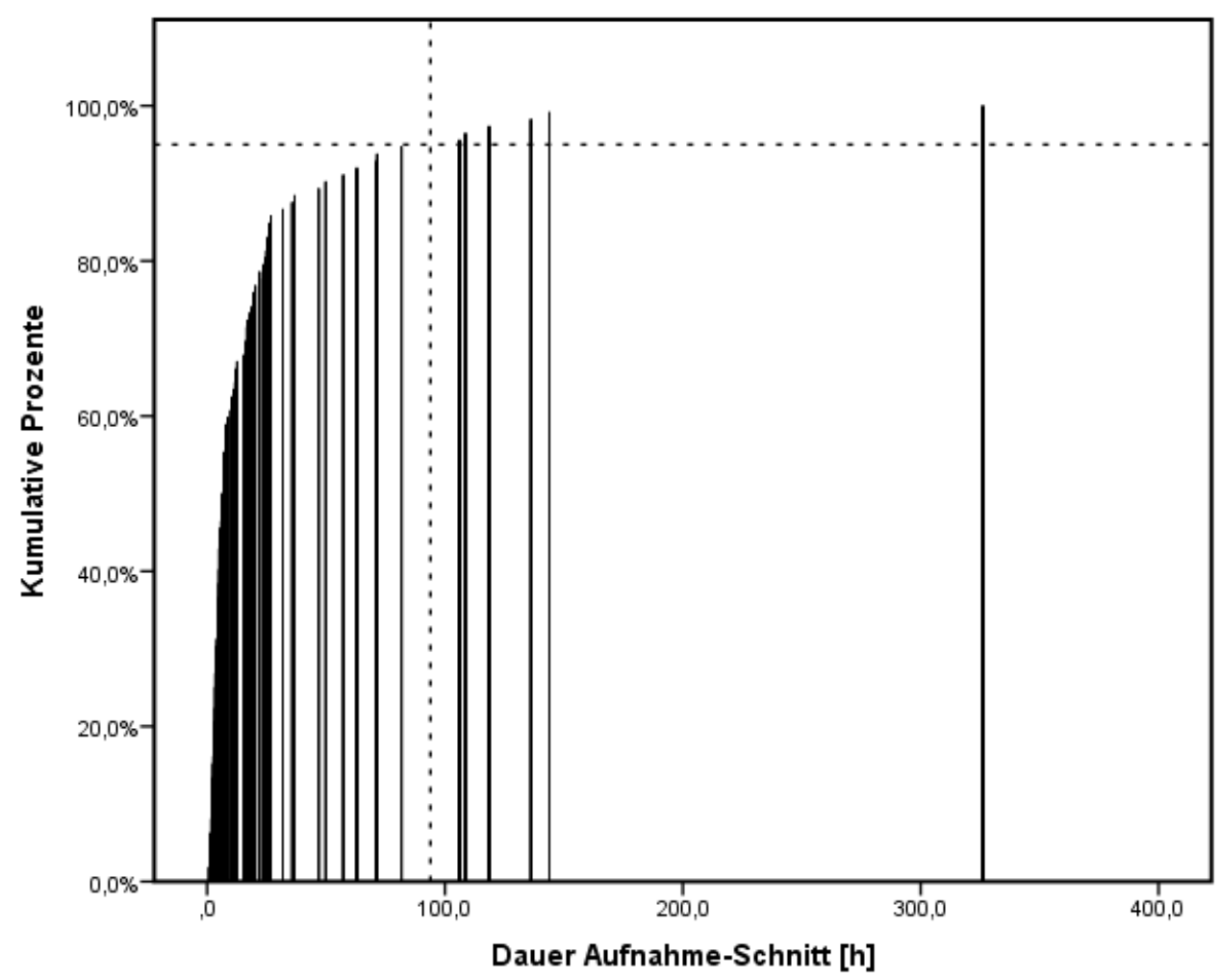

Abb. 2: Kumulative relative Häufigkeit einer perforierten Appendizitis in Abhängigkeit von Aufnahme-Schnitt-Dauer.

\subsubsection{Keine Peritonitis vs. Peritonitis}

Neben der Appendixperforation ist eine intraoperativ diagnostizierte Peritonitis ein Indikator für eine fortgeschrittene Appendizitis. Die Ergebnisse des Subgruppenvergleichs zwischen Patienten mit und ohne Peritonitis sind in Tabellen 20 und 21 zusammengefasst. Die Analyse bezieht sich auf 568 Patientenfälle, bei welchen eine intraoperative Beschreibung des Befundes einer Peritonitis erfolgte. Von diesen Patienten wiesen 102 Personen eine intraoperativ erkennbare Peritonitis auf, während 466 Patienten keine sichtbaren Anzeichen einer Peritonitis zeigten. 


\begin{tabular}{|c|c|c|c|}
\hline & keine Peritonitis & Peritonitis & $\mathbf{p}$ \\
\hline \multicolumn{4}{|l|}{ Demographische Daten } \\
\hline weibliches Geschlecht & $54,9 \%$ & $44,1 \%$ & 0,049 \\
\hline Alter $[y]$ & $26,6 \pm 17,2$ & $37,5 \pm 22,7$ & $<0,001$ \\
\hline \multicolumn{4}{|l|}{ Anamnestische Daten } \\
\hline Schmerzen $<4 \mathrm{~h}$ & $8,8 \%$ & $4,4 \%$ & 0,203 \\
\hline Schmerzen 4 bis $<8 \mathrm{~h}$ & $14,0 \%$ & $6,6 \%$ & 0,057 \\
\hline Schmerzen 8 bis $<16 \mathrm{~h}$ & $18,6 \%$ & $16,5 \%$ & 0,765 \\
\hline Schmerzen 16 bis $<24 \mathrm{~h}$ & $10,7 \%$ & $8,8 \%$ & 0,848 \\
\hline Schmerzen $\geq 24 \mathrm{~h}$ & $47,7 \%$ & $63,7 \%$ & 0,008 \\
\hline Kolik & $11,8 \%$ & $10,2 \%$ & 0,730 \\
\hline Dauerschmerz & $87,3 \%$ & $88,8 \%$ & 0,866 \\
\hline Schmerzwanderung & $19,5 \%$ & $29,6 \%$ & 0,030 \\
\hline Übelkeit & $44,4 \%$ & $53,5 \%$ & 0,119 \\
\hline Erbrechen & $29,3 \%$ & $45,5 \%$ & 0,003 \\
\hline Diarrhoe & $16,6 \%$ & $22,2 \%$ & 0,192 \\
\hline \multicolumn{4}{|l|}{ Klinische Untersuchung } \\
\hline rechter Unterbauch & $96,3 \%$ & $96,0 \%$ & 0,777 \\
\hline linker Unterbauch & $18,4 \%$ & $37,4 \%$ & $<0,001$ \\
\hline rechter Oberbauch & $8,8 \%$ & $17,2 \%$ & 0,017 \\
\hline periumbilikal & $14,0 \%$ & $23,2 \%$ & 0,032 \\
\hline epigastrisch & $7,9 \%$ & $18,2 \%$ & 0,004 \\
\hline McBurney-Zeichen & $33,3 \%$ & $23,2 \%$ & 0,056 \\
\hline Lanz-Zeichen & $21,9 \%$ & $18,2 \%$ & 0,498 \\
\hline Loslassschmerz & $32,5 \%$ & $30,3 \%$ & 0,723 \\
\hline Psoasschmerz & $18,4 \%$ & $14,1 \%$ & 0,383 \\
\hline Peritonismus & $13,2 \%$ & $29,3 \%$ & $<0,001$ \\
\hline Abwehrspannung & $31,6 \%$ & $46,5 \%$ & 0,007 \\
\hline \multicolumn{4}{|l|}{ Apparative Diagnostik } \\
\hline Sono o.p.B. & $57,9 \%$ & $43,9 \%$ & 0,013 \\
\hline Sono freie Flüssigkeit & $15,0 \%$ & $29,6 \%$ & 0,001 \\
\hline Sono Kokarde & $18,7 \%$ & $9,2 \%$ & 0,025 \\
\hline Sono freie Flüssigkeit und Kokarde & $7,3 \%$ & $8,2 \%$ & 0,831 \\
\hline Sono perityphlitischer Abszess & $1,1 \%$ & $9,2 \%$ & $<0,001$ \\
\hline CT durchgeführt & $2,1 \%$ & $16,7 \%$ & $<0,001$ \\
\hline Temperatur $\left[{ }^{\circ} \mathrm{C}\right]$ & $36,9 \pm 0,9$ & $37,0 \pm 1,0$ & 0,254 \\
\hline Temperatur $\leq 37^{\circ} \mathrm{C}$ & $65,1 \%$ & $59,6 \%$ & 0,434 \\
\hline Temperatur 37,1 bis $37,9^{\circ} \mathrm{C}$ & $19,5 \%$ & $17,3 \%$ & 0,848 \\
\hline Temperatur $\geq 38^{\circ} \mathrm{C}$ & $15,2 \%$ & $23,1 \%$ & 0,158 \\
\hline Leukozytenanzahl [x 109/l] & $12,6 \pm 5,0$ & $14,8 \pm 6,7$ & $<0,001$ \\
\hline CRP [mg/l] & $31,2 \pm 49,0$ & $81,4 \pm 92,4$ & $<0,001$ \\
\hline
\end{tabular}

Tabelle 20: Vergleich anamnestischer, klinischer und apparativ-diagnostischer Befunde von Patienten mit und ohne Peritonitis. Daten als prozentualer Anteil oder Mittelwert \pm Standardabweichung, $p$-Wert-Markierung bei $p<0,05$.

Patienten mit Peritonitis waren signifikant häufiger männlichen Geschlechts und darüber hinaus in der Regel älter.

Die Analyse der Schmerzdauer zeigte eine geringere Häufigkeit kurzer Beschwerdeintervalle bei Patienten mit im Vergleich zu Patienten ohne Peritonitis, wohingegen eine Schmerzanam- 
nese von mehr als 24 Stunden bei diesen Patienten signifikant häufiger war. Des Weiteren beschrieben sie deutlich häufiger Schmerzwanderung und Erbrechen. Patienten mit Peritonitis zeigten deutlich häufiger Druckschmerz in allen Bauchquadranten. Die typischen Appendizitiszeichen waren kein Indikator für das Vorliegen einer Peritonitis, jedoch wiesen Patienten mit Peritonitis signifikant häufiger Peritonismus und Abwehrspannung auf.

Die sonographische Untersuchung ergab deutlich häufiger pathologische Befunde wie freie Flüssigkeit und perityphlitische Abszessdarstellung bei letztlich diagnostizierter Peritonitis. Jedoch konnte eine pathologische Kokarde bei Peritonitis signifikant seltener dargestellt werden. Auffallend häufig wurde als Maßnahme der erweiterten diagnostischen Bildgebung auf eine CT-Untersuchung zurückgegriffen. Patienten mit Peritonitis zeigten im Vergleich zu Personen ohne Peritonitis bei Aufnahme signifikant höhere Leukozytenzahlen und CRP-Werte (Tabelle 20).

\begin{tabular}{|c|c|c|c|}
\hline & keine Peritonitis & Peritonitis & $\mathbf{p}$ \\
\hline \multicolumn{4}{|l|}{ Perioperatives Zeitmanagement } \\
\hline Aufnahme-Schnitt-Dauer [h] & $13,7 \pm 16,4$ & $22,3 \pm 41,5$ & 0,043 \\
\hline $\mathrm{ASD} \leq 4 \mathrm{~h}$ & $26,9 \%$ & $29,7 \%$ & 0,622 \\
\hline $\mathrm{ASD}>4 \mathrm{bis} \leq 8 \mathrm{~h}$ & $26,9 \%$ & $24,8 \%$ & 0,710 \\
\hline ASD $>8$ bis $\leq 12 \mathrm{~h}$ & $8,6 \%$ & $8,9 \%$ & 0,847 \\
\hline $\mathrm{ASD}>12$ bis $\leq 24 \mathrm{~h}$ & $21,6 \%$ & $10,9 \%$ & 0,013 \\
\hline ASD $>24$ bis $\leq 48 \mathrm{~h}$ & $11,9 \%$ & $12,9 \%$ & 0,738 \\
\hline $\mathrm{ASD}>48 \mathrm{~h}$ & $4,2 \%$ & $12,9 \%$ & 0,003 \\
\hline Operationsdauer [min] & $60,0 \pm 23,6$ & $78,5 \pm 37,1$ & $<0,001$ \\
\hline \multicolumn{4}{|l|}{ Operatives Vorgehen } \\
\hline offen & $34,3 \%$ & $39,2 \%$ & 0,361 \\
\hline laparoskopisch & $63,5 \%$ & $48,0 \%$ & 0,005 \\
\hline konvertiert & $2,1 \%$ & $12,7 \%$ & $<0,001$ \\
\hline \multicolumn{4}{|l|}{ Postoperative Daten } \\
\hline postoperative Verweildauer [d] & $4,3 \pm 5,4$ & $9,8 \pm 12,5$ & $<0,001$ \\
\hline Gesamtverweildauer [d] & $5,0 \pm 8,0$ & $10,7 \pm 12,6$ & $<0,001$ \\
\hline komplikationslos & $93,4 \%$ & $79,0 \%$ & $<0,001$ \\
\hline Wundheilungsstörung & $1,7 \%$ & $9,0 \%$ & 0,001 \\
\hline intraabdomineller Abszess & $0,4 \%$ & $1,0 \%$ & 0,448 \\
\hline Revisionsoperation & $2,8 \%$ & $11,0 \%$ & 0,001 \\
\hline Sonstige & $5,2 \%$ & $16,0 \%$ & 0,001 \\
\hline Re-Hospitalisation & $1,7 \%$ & $3,9 \%$ & 0,243 \\
\hline
\end{tabular}

Tabelle 21: Vergleich peri- und postoperativer Parameter von Patienten mit und ohne Peritonitis. Daten als prozentualer Anteil oder Mittelwert \pm Standardabweichung, $p$-Wert-Markierung bei $p<0,05$.

Die Auswertung der Aufnahme-Schnitt-Dauer zeigte eine signifikant längere Wartezeit zwischen Aufnahme und Operationsbeginn von etwa 9 Stunden bei Patienten mit Peritonitis. Dies ergab sich aus einem unverkennbar höheren Anteil an Patienten, die später als 48 Stunden nach 
Aufnahme einer Operation zugeführt wurden. Die Operationsdauer war bei Patienten mit Peritonitis signifikant länger und der Anteil von Konversions-Appendektomien deutlich erhöht.

Das Ausmaß des fortgeschrittenen intraoperativen Befundes spiegelte sich in der signifikant längeren stationären Verweildauer der Patienten mit Peritonitis wider. Diese zeigten zudem deutlich seltener einen komplikationslosen postoperativen Verlauf (Tabelle 21).

\subsection{Gegenüberstellung intraoperativer und histologischer Befund}

Bei insgesamt 643 Patienten standen sowohl intraoperativer als auch histologischer Befund einer Datenerhebung zur Verfügung und wurden zur Auswertung des Grades der Übereinstimmung zwischen Chirurgen und Pathologen in Bezug auf die Identifizierung einer akuten Appendizitis herangezogen.

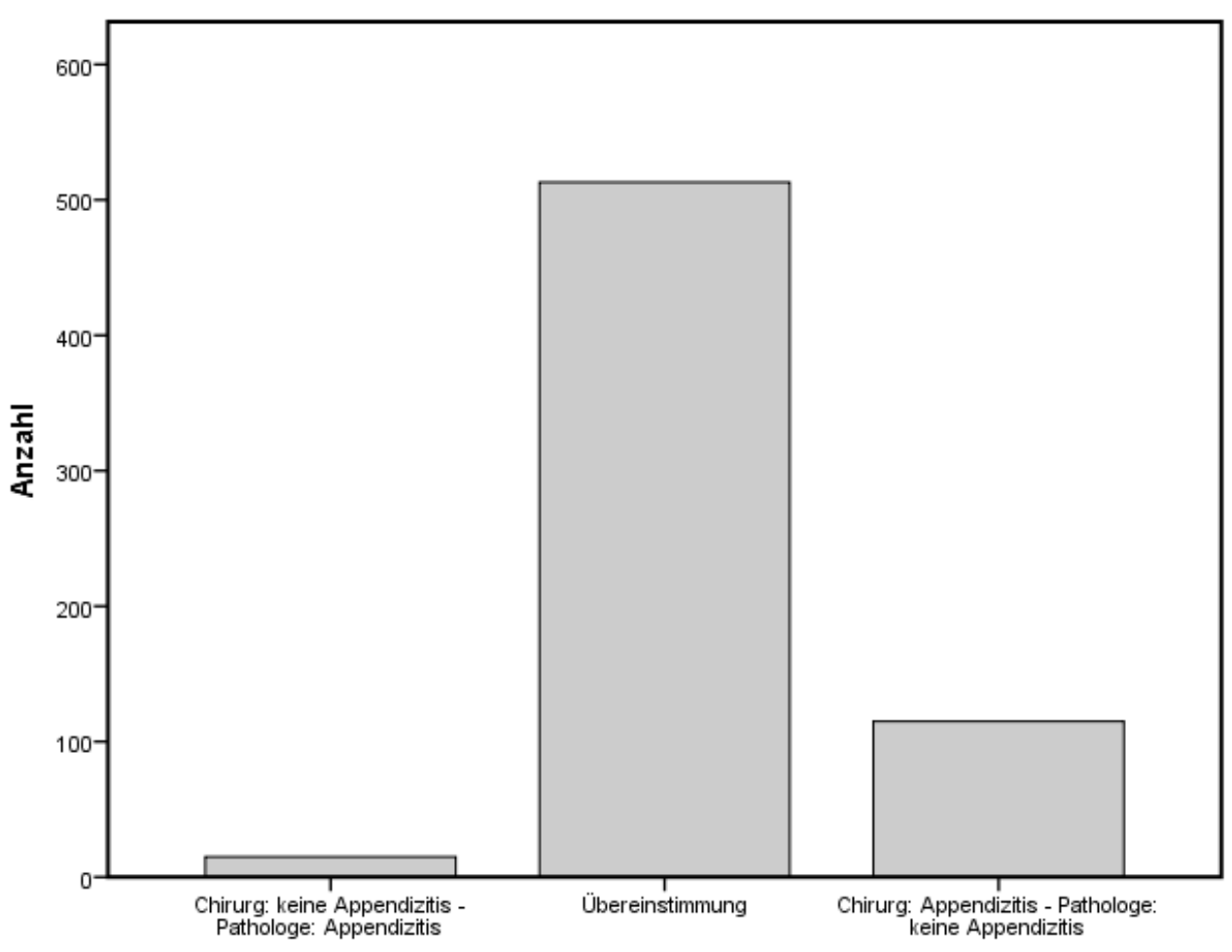

Abb. 3: Kongruenz des intraoperativen und histologischen Nachweises einer akuten Appendizitis.

Unabhängig von der Bestimmung des Schweregrades der Entzündungsreaktion kongruierten intraoperativer und histologischer Befund in 465 Fällen $(72,3 \%)$ in der Diagnose einer akuten Appendizitis und in 48 Fällen (7,5 \%) in der Beschreibung einer blanden Appendix. Der Übereinstimmungsgrad zwischen chirurgischer und pathologischer Bewertung lag somit bei 79,8\%. Die intraoperative Diagnose einer akut entzündeten Appendix konnte in 115 Fällen $(17,9 \%)$ histologisch nicht bestätigt werden. Umgekehrt waren allerdings bei 15 Patienten 
(2,3 \%) nach Entfernung einer intraoperativ reizlos erscheinenden Appendix histologisch trotzdem Zeichen einer akuten Entzündungsreaktion nachweisbar (Abbildung 3).

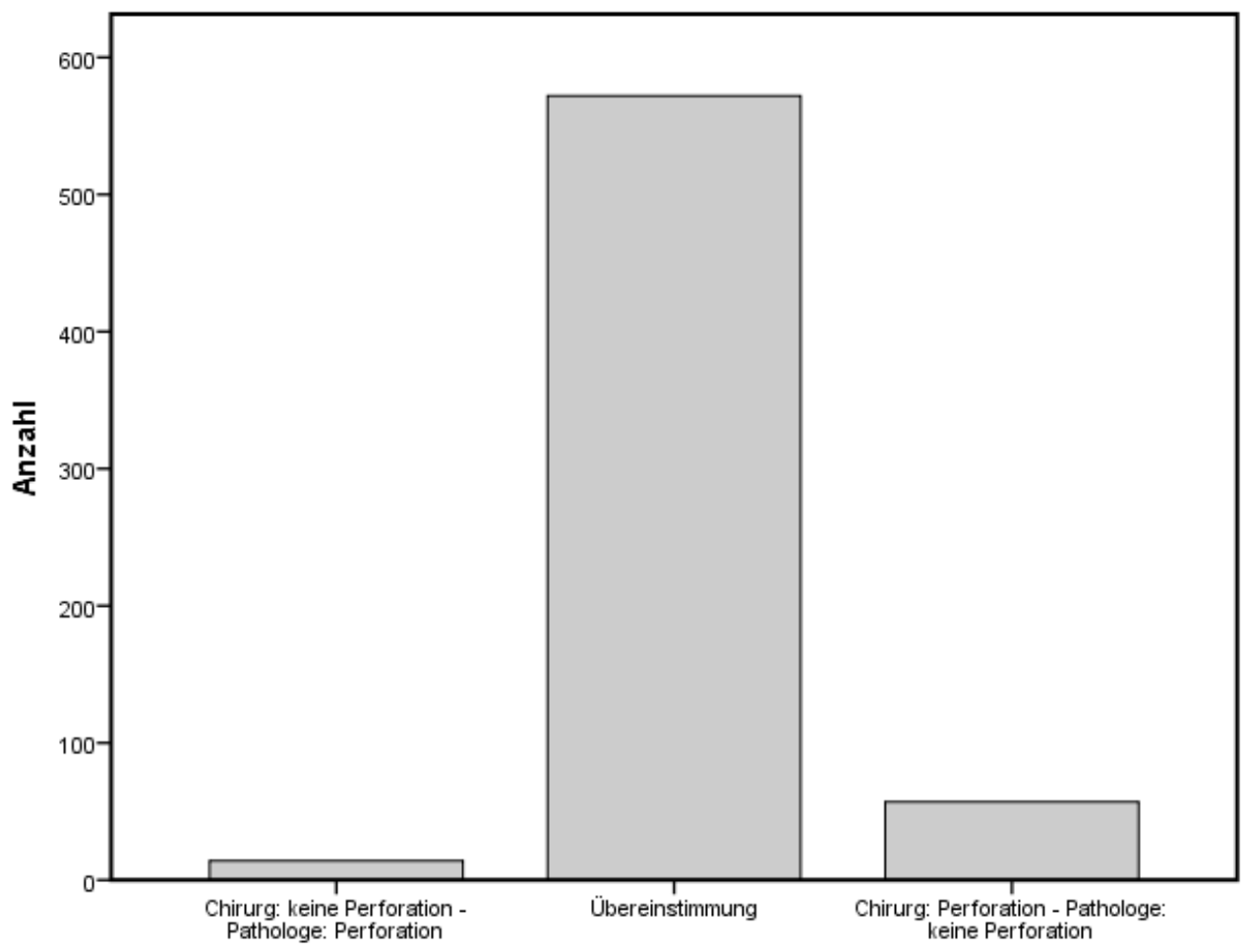

Abb. 4: Kongruenz des intraoperativen und histologischen Nachweises einer perforierten Appendizitis.

Abbildung 4 verdeutlicht die Ergebnisse der Diagnose einer perforierten Appendizitis. Eine Appendixperforation wurde in 14 Fällen $(2,2 \%)$ intraoperativ nicht beschrieben und erst nach Resektion histologisch diagnostiziert. Bei 57 Patienten (8,9\%) dokumentierte der Chirurg eine Perforation, die letztlich durch den Pathologen nicht bestätigt wurde. Der Grad der Übereinstimmung lag somit bei 88,9\% (Abbildung 4).

Um die Kongruenz des chirurgisch und pathologisch beurteilten Appendixzustandes hinsichtlich des Entzündungsstadiums zu visualisieren, wurde in Abbildung 5 die Darstellung in einem Bland-Altmann-Plot gewählt. Hierbei wurde die Differenz der Punktwerte als Maß der chirurgischen und pathologischen Einschätzung entsprechend der Beschreibung in Kapitel 2.4 gebildet und gegen den Mittelwert aufgetragen. Bei vollkommener Übereinstimmung stünden alle Werte auf der durchgezogenen Linie. Die Häufigkeit der jeweiligen Punktwertnennung wird durch die Dicke der Ringe im Diagramm repräsentiert. Schätzte der Chirurg das vorliegende Appendizitisstadium als fortgeschrittener als der Pathologe ein, so befinden sich die Punkte oberhalb der durchgezogenen Linie. Die Punkte unterhalb der Markierung stehen für eine schwerere Entzündungseinschätzung des Pathologen im Vergleich zum Chirurgen. 
Wie dem Diagramm zu entnehmen ist, lag die maximale Abweichung zu $95 \%$ in einem Punktwertintervall von etwa \pm 3 (95-\%-Konfidenzintervall). Es ist erkennbar, dass sowohl bei schwerem als auch bei eher unauffälligem Appendizitisbefund das Maß der Übereinstimmung höher war als bei mittlerem Schweregrad (Abbildung 5). Des Weiteren wurde bestätigt, dass die Differenz der Einschätzung des Schweregrades der akuten Appendizitis nicht signifikant $(\mathrm{p}=0,212)$ von „0“ unterschiedlich war, was gleichbedeutend mit einem nicht-signifikanten Unterschied in der Aussage von Chirurgen und Pathologen ist. Die Verteilung der Daten im BlandAltman-Plot zeigt bei gleichermaßen oberhalb wie unterhalb des 95-\%-Intervalls gelegenen Werten keinen „Bias“ der Datenerfassung, was durch eine nachfolgende lineare Regressionsanalyse bestätigt werden konnte.

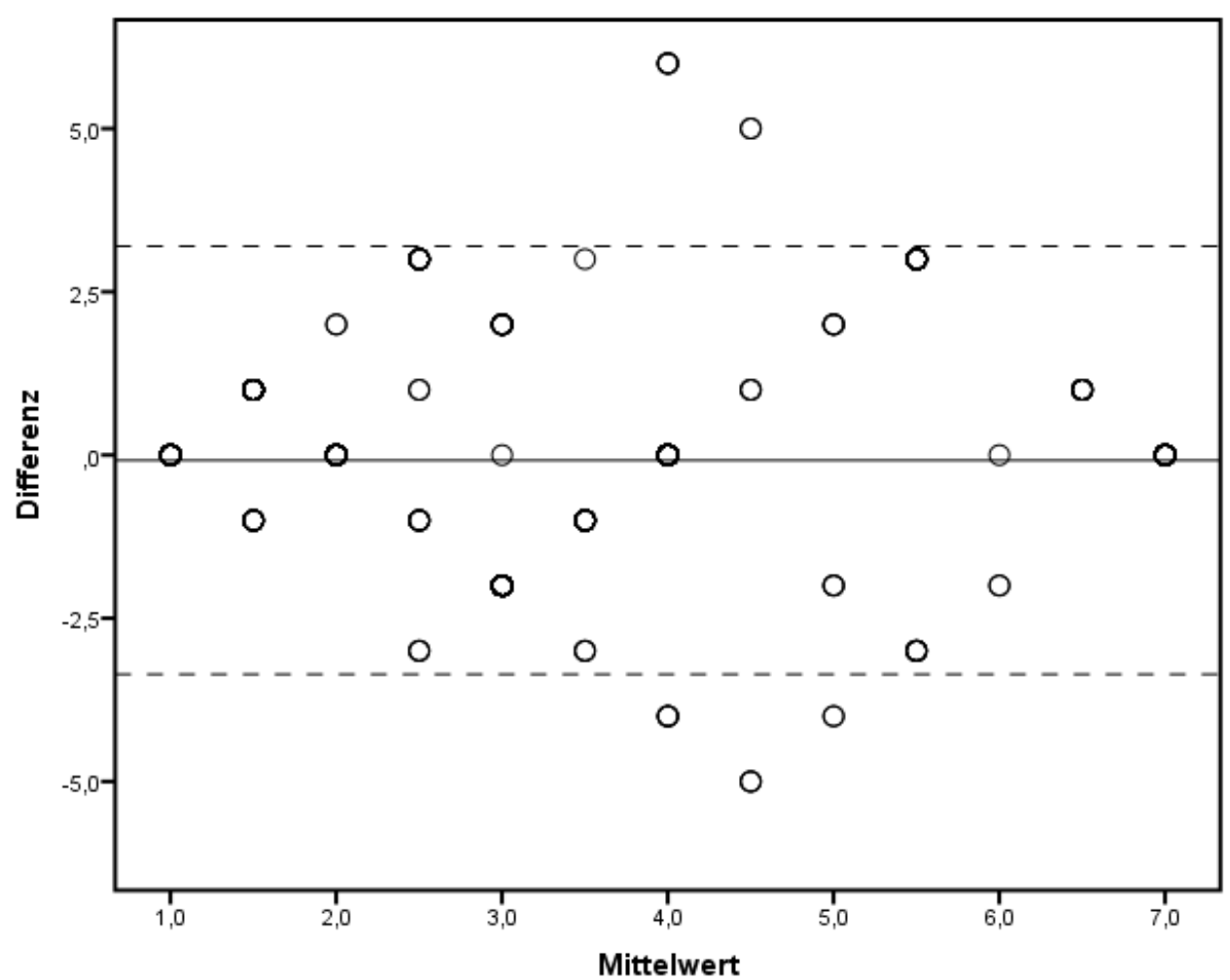

Abb. 5: Übereinstimmung der Einschätzung des Schweregrades der akuten Appendizitis zwischen Chirurg und Pathologe. 


\section{Diskussion}

\subsection{Präoperative Prädiktoren der akuten Appendizitis und des kom- plikationsbehafteten Krankheitsverlaufs}

Das häufige Auftreten der akuten Appendizitis begründet die Notwendigkeit einer sicheren diagnostischen Beurteilung eines jeden Chirurgen. Aufgrund der beschriebenen Variabilität der klinischen Manifestation ist eine korrekte Diagnose aber mitunter trotz der bekannten klassischen Anzeichen der akuten Appendizitis erschwert. Die Genauigkeit der präoperativen Appendizitisdiagnostik wird dabei in der Literatur mit etwa $80 \%$ beziffert, korrespondierend mit einer negativen Appendektomierate um $20 \%$ (Petroianu 2012). Die Betrachtung einzelner präoperativer Prädiktoren soll deren Aussagekraft und Stellenwert innerhalb der Appendizitisdiagnostik ermitteln. Die Vorhersagekraft dieser Parameter hinsichtlich des Schweregrades der Entzündung und eines möglichen komplikationsbehafteten postoperativen Verlaufs ist in der klinischen Routine ebenfalls von Interesse und wird im Folgenden in die Analyse des Stellenwertes der einzelnen Prädiktoren einbezogen.

Die Analyse der Ergebnisse dieser Untersuchung zeigt keine Geschlechtsspezifität der akuten Appendizitis. In zahlreichen Studien wird eine Dominanz des männlichen Geschlechts beschrieben (Addiss et al. 1990; Andersson et al. 1999; Laurell et al. 2013; Sadot et al. 2013), welche in diesem Kollektiv nicht bestätigt werden konnte. Eine Appendektomie trotz letztlich histologisch nicht manifester akuter Entzündung wurde in Kongruenz mit Literaturangaben jedoch bei deutlich mehr Frauen als Männern durchgeführt (Junginger und Küchle 1998; Laurell et al. 2013). Dieses Phänomen ist mit der Anzahl der Differentialdiagnosen, welche die Symptome einer akuten Appendizitis imitieren können, bei weiblichen im Vergleich zu männlichen Patienten zu erklären. In der Literatur werden negative Appendektomieraten von $9 \%$ (Piper et al. 2008) bis 25 \% (Andersson et al. 1999) genannt, welche mit den Ergebnissen dieser Untersuchung vereinbar sind. Mit einem durchschnittlichen Patientenalter von 28 Jahren und einem Häufigkeitsgipfel zwischen dem 10. und 29. Lebensjahr zeigen die Patientendaten einen breiteren maximalen Manifestationsgipfel als von Addiss et al. beschrieben (Addiss et al. 1990). Auch der Ausschluss negativer Appendektomien modifizierte das Durchschnittsalter des Gesamtkollektivs nicht. Analog beschreiben Sahm et al. einen Anstieg des Durchschnittsalters von Appendizitispatienten im Zeitraum von 1988 bis 2009. Die demographische Entwicklung Deutschlands mit sinkender Geburtenrate könnte eine Erklärung für diese Altersverschiebung bieten (Sahm et al. 2013), wobei eine Disposition der akuten Appendizitis im Kindes- und jungen Erwachsenenalter erkennbar bleibt. Ebenso kann spekuliert werden, ob junge Patienten mit unklaren abdo- 
minellen Beschwerden eher ein städtisches Krankenhaus als die Universitätsklinik aufsuchen und somit die Durchführung dieser Studie an einer Universitätsklinik als Erklärung für das Phänomen der untypischen Altersverteilung des Kollektivs dienen kann. Hohes Alter und männliches Geschlecht waren im untersuchten Kollektiv häufiger mit schwerem Entzündungszustand und komplikativem postoperativem Aufenthalt assoziiert. Zahlreiche Publikationen berichten übereinstimmend von vermehrt fortgeschrittenen Appendizitisbefunden bei Männern im Vergleich zu Frauen (Sadot et al. 2013; Obinwa et al. 2015). Hinsichtlich einer Altersdisposition divergieren die Quellen. In Abhängigkeit vom untersuchten Patientenkollektiv berichten einzelne Autoren von vermehrt jungen (Obinwa et al. 2015) oder älteren Patienten (Andersson et al. 1999; Sadot et al. 2013) mit Appendixperforation. Mit einem Anteil histologisch bestätigter Appendixperforationen von unter $10 \%$ liegt die Perforationsrate des Gesamtkollektivs unter dem in Publikationen beschriebenen Anteil perforierter Appendizitiden von bis zu $20 \%$ (Zielke 2002). Nur in einer Studie konnte eine ausgesprochen niedrige Perforationsrate von 3,7\% nachgewiesen werden (Junginger und Küchle 1998). Die niedrige Perforationsrate des Kollektivs in dieser Studie mag Ausdruck der im hiesigen Zentrum herrschenden Philosophie einer großzügigen Indikationsstellung zur Exploration und Appendektomie sein.

Aus der Betrachtung der Schmerzdauer lässt sich im untersuchten Patientenkollektiv wie auch in den Ergebnissen von Laurell et al. kein sicherer Prädiktor einer akuten Appendizitis ermitteln (Laurell et al. 2013). Mittlere Beschwerdedauern von 8 bis 16 Stunden zeigten in Übereinstimmung mit Andersson et al. eine gewisse Vorhersagekraft für eine akute Appendizitis (Andersson et al. 1999). Länger als 24 Stunden bestehende Schmerzen verminderten die Wahrscheinlichkeit einer akuten Appendizitis zwar etwas, jedoch beklagte fast die Hälfte der Patienten mit letztlich bestätigter akuter Entzündung derart lang andauernde Beschwerden. Dieser Sachverhalt ist mit dem klassischen Symptomverlauf der akuten Appendizitis zu erklären. Typischerweise durchlebt der Patient zunächst diffuse, jedoch leichte viszerale Bauchschmerzen, die nicht die unmittelbare Notwendigkeit einer ärztlichen Konsultation oder gar stationären Vorstellung nahelegen. Erst nach Stunden erfolgt die Intensivierung und Verlagerung der Beschwerden in den rechten Unterbauch im Sinne eines somatischen Schmerzes, die den Patienten zur Inanspruchnahme medizinischer Hilfe veranlasst. Zwei Publikationen nennen einen Zeitraum von 12 bis 48 Stunden, in welchem sich ein Großteil der Patienten in der Regel zur ärztlichen Vorstellung entschließt (Wagner et al. 1996; Boenigk et al. 2012). Andere Autoren postulieren eine Abnahme der Wahrscheinlichkeit für eine akute Appendizitis nach einer Symptomdauer von über 48 Stunden (Andersson et al. 1999; Laurell et al. 2013), wobei ein Vergleich mit den Daten dieser Untersuchung aufgrund der fehlenden Unterteilung der Patientengruppe mit länger als 24 Stunden bestehenden Beschwerden nicht möglich ist.

Übelkeit, Erbrechen und Schmerzwanderung wurden übereinstimmend mit anderen Publikationen vermehrt bei korrekt diagnostizierter akuter Appendizitis angegeben (Andersson et al. 
1999; Laurell et al. 2013), wobei die Abwesenheit dieser Symptome, wie den Ergebnissen zu entnehmen ist, keinesfalls den Ausschluss einer akuten Appendizitis zulässt. Während Schmerzwanderung, Übelkeit und Erbrechen im untersuchten Patientenkollektiv ebenfalls prädiktiv für einen fortgeschrittenen Appendizitisbefund waren, berichten Obinwa et al. von einer unzureichenden Aussagekraft dieser Parameter in der Differenzierung von Appendizitisstadien (Obinwa et al. 2015). Zudem konnte keine Assoziation mit einer vermehrten Komplikationsrate insbesondere für die beiden letztgenannten Parameter im untersuchten Patientenkollektiv nachgewiesen werden.

Trotz des hohen Stellenwertes der klinischen Untersuchung innerhalb der Appendizitisdiagnostik ist ihre Aussagekraft und Zuverlässigkeit den Ergebnissen dieser Studie zufolge eingeschränkt. Dies räumen auch Andersson et al. ein, welche ihr dennoch einen höheren Stellenwert als der alleinigen Anamneseerhebung zuerkennen (Andersson et al. 1999). Im Kollektiv konnte der Nachweis einzelner positiver Appendizitiszeichen eine akute Appendizitis weder mit absoluter Sicherheit prognostizieren noch ausschließen. Nur Peritonismus wurde deutlich häufiger bei Patienten mit akuter Appendizitis nachgewiesen. Analog messen Andersson et al. den Anzeichen peritonealer Reizung einen hohen Stellenwert in der Appendizitisdiagnostik bei (Andersson et al. 1999). Insbesondere Abwehrspannung, Loslassschmerz und Schmerzen im rechten Unterbauch zeigen in anderen Publikationen eine deutliche Assoziation mit einer akuten Appendizitis (Laurell et al. 2013; Obinwa et al. 2015). Die Ergebnisse dieser Untersuchung konnten das nicht bestätigen, auch wenn Loslassschmerz und Abwehrspannung im direkten Vergleich mit anderen typischen Appendizitiszeichen höhere Treffsicherheiten aufwiesen. Schmerzen im rechten Unterbauch wurden mit gleicher Häufigkeit bei Patienten mit und ohne akute Appendizitis diagnostiziert. Daraus lässt sich der Stellenwert dieses „Leitsymptoms“ als notwendige, jedoch nicht hinreichende Bedingung für die Diagnose einer akuten Appendizitis ableiten, welche zur Erwägung der Verdachtsdiagnose einer akuten Appendizitis führen sollte. Die Ergebnisse dieser Untersuchung decken sich mit der Schlussfolgerung von Petroianu, nach der typische Appendizitiszeichen in der Regel bei weniger als 40 \% der Appendizitispatienten nachgewiesen werden können (Petroianu 2012). Letztlich sollte ein fehlender Nachweis der klassischen Appendizitiszeichen demnach nicht zum sofortigen Ausschluss der Diagnose einer akuten Appendizitis verleiten. Innerhalb der Patienten mit perforierter Appendizitis war in dieser Untersuchung eine Tendenz zu vermehrt ubiquitärem Abdominalschmerz mit häufigen Palpationsbeschwerden in allen Bauchquadranten sowie Peritonismus und Abwehrspannung als Symptome des akuten Abdomens erkennbar. Analog beschreiben Ditillo et al. vermehrte Abwehrspannung und Schmerzen außerhalb des rechten Unterbauches im Zusammenhang mit perforierten Appendizitiden (Ditillo et al. 2006). Ähnliche, jedoch statistisch nicht signifikante Ergebnisse zeigte in der vorliegenden Studie die klinische Untersuchung der Patienten mit postoperativen Komplikationen. Auch Obinwa et al. konnten anhand der klinischen Untersuchung keinen eindeutigen Prädiktor für 
postoperative Komplikationen nachweisen (Obinwa et al. 2015). Im untersuchten Kollektiv wurden typische Appendizitiszeichen seltener bei Patienten mit komplikativem als mit postoperativ regulärem Verlauf nachgewiesen, wobei vergleichbare Ergebnisse in der Literaturrecherche nicht auffindbar waren. Möglicherweise führt ein fehlender Nachweis von Appendizitiszeichen zu einer zögerlichen Operationsindikationsstellung, welche eine Progression der Entzündung und in der Folge einen vermehrt komplikativen Genesungsverlauf bedingen kann.

Ein Vergleich der bildgebenden Diagnostik zeigt eine eindeutige Bevorzugung der Abdomensonographie gegenüber der Computertomographie an der Universitätsmedizin Göttingen. Damit wird der Einordnung der Ultraschalluntersuchung als „Schnittbildverfahren der ersten Wahl“ (Binnebösel et al. 2009, S. 579) zugestimmt. Bei mehr als der Hälfte der Appendizitispatienten konnte sonographisch kein pathologischer Befund nachgewiesen werden und auch in der Literatur wird eine geringe Korrelation zwischen unauffälligem Sonographiebefund und intraoperativer Appendixbeurteilung beschrieben (Khanal et al. 2008). Zielke et al. konnten des Weiteren eine starke Behandlerabhängigkeit der korrekten Darstellung einer pathologisch veränderten Appendix belegen (Zielke et al. 2001). Die Aussagekraft eines unauffälligen sonographischen Befundes bleibt somit eingeschränkt und erfordert eine differenzierte Beurteilung, die nicht zum definitiven Ausschluss einer akuten Appendizitis führen sollte. Innerhalb der sonographischen Bildgebung war der Nachweis einer pathologischen Kokarde (Franke et al. 1999) oder eines perityphlitischen Abszesses mit hoher Sicherheit für die korrekte Diagnose einer akuten Appendizitis vergesellschaftet, doch auch hier konnte aus der nicht erfolgten Darstellung keinesfalls der Ausschluss eines akuten Entzündungsgeschehens geschlussfolgert werden. Anhand dieser Ergebnisse lässt sich in Analogie mit Literaturangaben (Zielke 2002) resümieren, dass bei Darstellung eines der oben genannten pathologischen Befunde von einem akut entzündlichen Geschehen ausgegangen und die Indikation zur Appendektomie nahegelegt werden kann, während die Darstellung eines unauffälligen sonographischen Befundes nicht zur Verwerfung der Verdachtsdiagnose einer akuten Appendizitis führen darf. Postoperative Komplikationen konnten im untersuchten Kollektiv nur mit sonographischem Nachweis eines perityphlitischen Abszesses assoziiert werden. Hingegen schlug sich eine Appendixperforation häufig in darstellbarer freier Flüssigkeit und vermehrter Abszessdarstellung sowie seltenerem Nachweis eines unauffälligen Befundes nieder.

Als alternatives Bildgebungsverfahren zeigt die Computertomographie eine geringe Untersucherabhängigkeit, hohe Genauigkeitswerte in der Diagnosestellung der akuten Appendizitis (Binnebösel et al. 2009) und vermag bei richtiger Indikationsstellung die negative Appendektomierate zu senken (Zoarets et al. 2014). Der beschränkte Einsatz innerhalb des Gesamtkollektivs $(4,3 \%)$ ist mit einer sehr strengen Indikationsstellung zu erklären, um eine unnötige Strahlenexposition der Patienten zu vermeiden. Mit dieser Anwendungshäufigkeit übereinstimmend nennen Sahm et al. eine CT-Untersuchungsrate von 7,7 \% in einer 2013 veröffentlichten deut- 
schen Studie (Sahm et al. 2013). Während die Computertomographie in Deutschland daher nicht als standarddiagnostisches Verfahren angesehen wird, findet sie in den USA zunehmende Verbreitung in der Appendixbeurteilung (Reich et al. 2011). In der Diagnostik der akuten Appendizitis spielte die CT-Untersuchung in diesem Kollektiv eine untergeordnete Rolle und wurde letztlich nur bei Patienten eingesetzt, bei denen die üblichen klinischen und diagnostischen Verfahren keine zufriedenstellende Beurteilung des Patienten erlaubten, allerdings ernsthafte Symptomatik und Befundkonstellation eine zügige Diagnosestellung erforderlich machten. Dies unterstreicht den Stellenwert dieses bildgebenden Verfahrens im Falle der dringlichen Diagnosestellung nach Ausschöpfung der Möglichkeiten der Standarddiagnostik (Reich et al. 2011).

Die untersuchten laborchemischen Entzündungsparameter eigneten sich sowohl in der vorliegenden Untersuchung als auch in anderen Publikationen als Prädiktoren einer akuten oder perforierten Appendizitis (Sadot et al. 2013) sowie postoperativer Komplikationen (Obinwa et al. 2015). Die Leukozytenzahlen bei akuter Appendizitis im Vergleich zu fortgeschrittenem Entzündungsbefund mit Appendixperforation waren in vergleichbarem Maße erhöht, was auf die große Vorhersagekraft eines akuten Entzündungsgeschehens schließen lässt. Tendenziell wird eine Leukozytose durch einen schweren Entzündungsverlauf verstärkt, ohne jedoch sichere Rückschlüsse auf das Ausmaß des Entzündungsfortschritts zuzulassen (Grönroos und Grönroos 1999). Ergänzend hierzu korrelierte die Höhe des CRP-Wertes entsprechend der dargestellten Ergebnisse und der Beobachtungen anderer Autoren besser mit dem Fortschritt des Appendizitisbefundes (Chung et al. 1996; Sack et al. 2006). In Übereinstimmung mit anderen Publikationen war Fieber mit postoperativen Komplikationen (Obinwa et al. 2015) und fortgeschrittenem Appendizitisbefund assoziiert (Sadot et al. 2013)

Obwohl einzelne präoperative Prädiktoren für die Diagnose einer akuten Appendizitis und die Prognose des postoperativen Verlaufs existieren, ist ihre Aussagekraft innerhalb der Diagnostik eingeschränkt. Die Studienlage divergiert hinsichtlich der Frage nach den wichtigsten präoperativen Parametern, jedoch herrscht auch in Kongruenz mit den Ergebnissen der vorliegenden Datenanalyse Einigkeit darüber, dass es keinen einzelnen sicheren Prädiktor der akuten Appendizitis gibt. Vielmehr bleibt die klinische Untersuchung in Zusammenschau mit der Anamnese die Basis der Appendizitisdiagnostik, welche sinnvollerweise um sonographische Bildgebung, laborchemische Blutuntersuchung und Körpertemperaturmessung ergänzt werden sollte. Die Kombination typischer Appendizitiszeichen besitzt gemäß Laurell et al. eine erhöhte Aussagekraft (Laurell et al. 2013) und insbesondere sonographischer und laborchemischer Befund sollten nicht losgelöst vom klinischen Erscheinungsbild des Patienten bewertet, sondern als ergänzendes Diagnostikum verstanden werden. Da der Diagnosestellung aber letztlich die individuelle klinische Erfahrung des Chirurgen zugrunde liegt, zeigen sich selbst innerhalb eines Krankenhauses mit eindeutigen chirurgischen Leitlinien behandlerabhängige Unterschiede der negativen Appendektomie- und postoperativen Komplikationsrate (Junginger und Küchle 1998). 
Wichtige Parameter, die die korrekte Diagnose einer akuten Appendizitis erhärten können, sind dieser Untersuchungen zufolge mittlere Schmerzdauer, Übelkeit, Erbrechen, Schmerzwanderung, Peritonismus, sonographische Darstellung einer pathologischen Kokarde oder eines perityphlitischen Abszesses, Leukozytose und CRP-Anstieg. Zwar ist die Studienlage zu präoperativen Prädiktoren eines fortgeschrittenen Appendizitisbefundes und postoperativer Komplikationen mangelhaft, wie Ditillo et al. bereits 2006 feststellten (Ditillo et al. 2006), doch gewinnt gemäß der Ergebnisse der vorliegenden Untersuchung ein komplikativer postoperativer Verlauf an Wahrscheinlichkeit bei hohem Alter, männlichem Geschlecht, ubiquitärem Abdominalschmerz, sonographischer Darstellung eines perityphlitischen Abszesses, Fieber, Leukozytose und deutlichem CRP-Anstieg. Eine progrediente Symptomatik lässt zwar auf einen fortgeschrittenen Appendizitisbefund schließen und erhöht die Wahrscheinlichkeit eines postoperativ komplikationsbehafteten Verlaufs, doch ist nicht jede Appendixperforation automatisch mit postoperativen Komplikationen verbunden. Entsprechend kann vermutet werden, dass neben einem fortgeschrittenen Entzündungsbefund auch andere Faktoren wie zum Beispiel patienteneigene Faktoren und Komorbiditäten (Boenigk et al. 2012) sowie Operationstechnik und Erfahrung des Operateurs die postoperative Komplikationsrate beeinflussen.

\subsection{Einfluss der Aufnahme-Schnitt-Dauer auf den Schweregrad der Appendizitis und Einfluss eines fortgeschrittenen Befundes auf den postoperativen Verlauf}

Traditionellerweise ist die Diagnose einer akuten Appendizitis gleichzusetzen mit einer Indikation zur sofortigen chirurgischen Intervention (Peiper 2006). Mehr als die Hälfte der Patienten mit der Verdachtsdiagnose einer akuten Appendizitis wurden in diesem Kollektiv innerhalb von 8 Stunden nach Aufnahme operiert. In einer vergleichbaren Studie von Piper et al. wurden lediglich $21 \%$ der Patienten nach maximal 8 Stunden einer Operation zugeführt. Die durchschnittliche Aufnahme-Schnitt-Dauer blieb dabei mit 11,6 Stunden unter der in der vorliegenden Untersuchung gemessenen Zeit von 14,7 Stunden, da Patienten mit um mehr als 24 Stunden verzögerter Operation in der zitierten Studie nicht aufgeführt wurden (Piper et al. 2008). In einer Untersuchung von Hornby et al., in welche auch Aufnahme-Schnitt-Dauern von mehr als 24 Stunden einflossen, wurde eine mit den Ergebnissen dieses Patientenkollektivs übereinstimmende durchschnittliche stationäre Wartezeit von 14,4 Stunden publiziert (Hornby et al. 2014). Bei Betrachtung der dargestellten Ergebnisse ist erkennbar, dass Patienten mit fortgeschrittenem Appendizitisbefund eine durchschnittlich längere Aufnahme-Schnitt-Dauer als Patienten mit leichtem Entzündungszustand hatten. Es verstrich deutlich mehr Zeit bis zum Operationsbeginn 
bei Patienten mit Appendixperforation (20,5 \pm 40,3 Stunden) und Peritonitis (22,3 \pm 41,5 Stunden). Die erhöhte Standardabweichung signalisiert hierbei eine zunehmende Streubreite der Patientendaten im Vergleich zu Patienten ohne Peritonitis oder perforierte Appendizitis. So wurden im direkten Vergleich der Patientengruppen Patienten mit fortgeschrittenem Entzündungszustand sowohl vermehrt schnell innerhalb von 4 Stunden als auch verzögert nach 48 Stunden einer Operation zugeführt. Gleichzeitig war die von Patienten mit fortgeschrittenem Appendizitisbefund beschriebene Symptomdauer in Übereinstimmung mit den Ergebnissen von Temple et al. länger im Vergleich zu Patienten ohne Perforation und Peritonitis (Temple et al. 1995). Eine zeitliche Abhängigkeit der Appendizitisprogression wurde bereits 1886 durch Fitz postuliert und begründet heutzutage noch immer die weitreichende Forderung nach einer zeitnahen chirurgischen Intervention (Abou-Nukta et al. 2006). Andere aktuelle Untersuchungen äußern hingegen die Vermutung einer eigenen Pathophysiologie der Appendixperforation im Vergleich zu leichteren Appendizitisstadien (Livingston et al. 2007; Hornby et al. 2014). Es drängt sich demnach die Frage auf, ob eine frühzeitige Operation die Perforationsrate überhaupt positiv zu beeinflussen vermag oder ob diese eher im zeitlichen Zusammenhang mit einer vermehrt verzögerten Patientenvorstellung steht.

Die durchgeführte Ergebnisanalyse lässt vermuten, dass ein Großteil der Appendixperforationen im Kollektiv bereits bei Patientenaufnahme bestand. Lässt man zunächst die ausgesprochen spät operierten Patienten (> 48 Stunden) außer Acht, so wird deutlich, dass sich das perioperative Zeitmanagement der Patienten mit und ohne fortgeschrittenen Entzündungszustand kaum unterschied und Patienten mit Perforation und Peritonitis tendenziell sogar schneller operativ versorgt wurden als Patienten mit leichtem Entzündungsbefund. Etwa 58 \% der Patienten mit perforierter Appendizitis wurden nach maximal 8 Stunden - und damit dem Grundsatz des Appendizitismanagements entsprechend „zeitnah“ - operiert, zeigten aber trotzdem ein deutlich fortgeschrittenes Krankheitsstadium. Vergleichbare Ergebnisse liefern andere Studien, nach denen Patienten mit Perforation und Abszessbildung kein anderes chirurgisches Zeitmanagement erfuhren als Patienten mit leichten Appendizitisstadien (Piper et al. 2008; Bhangu 2014; Drake et al. 2014).

In der Regel führten bei Patienten mit perforierter Appendizitis eindrucksvolle Symptomatik und eindeutige klinische Befunde zur zügigen Operationsindikationsstellung. Die zeitliche Verzögerung bei den sehr spät operierten Patienten ( $>48$ Stunden) ist im untersuchten Patientenkollektiv mit einer uneindeutigen klinischen Präsentation zu erklären. In einigen Fällen begründet demnach die späte Manifestation einer Appendizitis mit dann vermehrt fortgeschrittenem intraoperativem Entzündungsbefund eine verzögerte Indikationsstellung. Gupta et al. bestätigen diese Erklärung für einen verzögerten Operationsbeginn und empfehlen bei Patienten mit uneindeutiger Symptomatik eine abwartende chirurgische Haltung bei gleichzeitig engma- 
schiger Patientenüberwachung, um eine korrekte Indikationsstellung zu gewährleisten (Gupta et al. 2010).

Die klinische Bedeutung der Frage nach der Richtigkeit eines gering verzögerten Operationsbeginns ist insbesondere anhand der Debatte um die Sicherheit notfallmäßiger nächtlicher Operationen erkennbar. In einer Untersuchung von Bhangu wurden $20 \%$ der Appendektomien zwischen Mittenacht und 8 Uhr morgens durchgeführt (Bhangu 2014). In einer anderen Studie erfolgten 57 \% der Operationen außerhalb der regulären Operationszeiten in den späten Abendund frühen Morgenstunden (Abou-Nukta et al. 2006). Eine leichte Verzögerung des chirurgischen Eingriffs könnte demnach in vielen Fällen eine Operation zu regulären Arbeitszeiten ermöglichen. Dies ist nach Eastridge et al. insbesondere vor dem Hintergrund einer erhöhten chirurgischen Fehlerquote bei Laparoskopien unter Schlafmangel erwägenswert (Eastridge et al. 2003). Somit könnte ein bewusstes Verschieben von Appendektomien in das operative Routineprogramm zur Reduktion der durch nächtliches Operieren bedingten Komplikationen führen.

Eine zeitliche Abhängigkeit der Entwicklung einer fortgeschrittenen Appendizitis ist in $\mathrm{Pu}$ blikationen beschrieben (Ditillo et al. 2006; Sadot et al. 2013). Anhand eines Vergleiches des untersuchten Patientenkollektivs mit Patienten, welche im selben Untersuchungszeitraum ausschließlich stationär überwacht, jedoch keiner operativen Therapie zugeführt wurden, könnte der zeitliche Verlauf dieser Entzündungsreaktion überprüft werden. Entsprechende Daten sind jedoch anhand der vorliegenden Datenerhebung nicht verfügbar. Zahlreiche Autoren schlussfolgern trotz des genannten Zusammenhangs eines Operationsaufschubes mit schweren Appendizitisformen, dass kurze Verzögerungen der Aufnahme-Schnitt-Dauer von bis zu 24 Stunden die Rate an fortgeschrittenen Appendizitisbefunden mit Perforation und Abszessbildung nicht negativ beeinflussen (Abou-Nukta et al. 2006; Clyde et al. 2008; Bhangu 2014), da der Entzündungsfortschritt eher durch verspätete Patientenvorstellung als durch krankenhausinterne präoperative Verzögerungen beeinflusst wird (Ditillo et al. 2006; Clyde et al. 2008; Sadot et al. 2013; Drake et al. 2014). Eine „erlaubte“ kurzzeitige Verzögerung des Operationsbeginns könnte demnach neben der beschriebenen Verlegung zahlreicher Appendektomien in das reguläre Krankenhaustagesgeschäft ein Zeitfenster für erweiterte präoperative Diagnostik bereitstellen, welche eine erhöhte Rate korrekter Operationsindikationen zur Folge haben könnte. Diese Hypothese bedarf jedoch der Überprüfung in weiteren klinischen Untersuchungen. Andererseits halten gerade aufgrund des beschriebenen Zeiteinflusses auf den Entzündungsfortschritt einige Autoren eine Operationsverzögerung für unsicher, da Perforationen, die erst nach stationärer Patientenaufnahme auftreten, im Einflussbereich des Chirurgen liegen und verhindert werden sollten (Ditillo et al. 2006; Sadot et al. 2013). Einschränkend bleibt zu erwähnen, dass in der Mehrzahl der Studien, welche eine präoperative Verzögerung favorisieren, die stationäre Patientenaufnahme mit der Einleitung einer antibiotischen Therapie einherging. Da dieses Verfahren in der Universitätsmedizin Göttingen keine Anwendung findet, um den Verlauf der charakteristischen Appendi- 
zitissymptomatik nicht zu verschleiern, sind die Schlussfolgerungen jener Publikationen nur eingeschränkt auf das Universitätsklinikum Göttingen übertragbar.

Unter der Zielsetzung eine „Handlungsanweisung“ des chirurgischen Appendizitismanagements im klinischen Alltag zu formulieren, muss nicht nur die Frage des Zeiteinflusses auf den Fortschritt der Erkrankung erörtert werden. Auch die weiterführende Betrachtung des Einflusses von Perforation und Peritonitis auf den Genesungsverlauf der Patienten ist hierbei von Interesse. Mit 9,3\% befindet sich die Komplikationsrate des Gesamtkollektivs in Übereinstimmung mit anderen Literaturangaben, in welchen postoperative Komplikationen bei $6 \%$ (Bijnen et al. 2003) bis 15 \% (Junginger und Küchle 1998) der Patienten beschrieben werden. Die Häufigkeit von Wundheilungsstörungen war mit $3 \%$ etwas niedriger als in anderen Publikationen (Clyde et al. 2008). Allerdings mussten sich Patienten etwas häufiger einer Revisionsoperation unterziehen (Bijnen et al. 2003). Während sich der postoperative stationäre Aufenthalt von Patienten mit akuter Appendizitis im Vergleich zu Patienten mit negativer Appendektomie nicht signifikant unterschied, suggerieren die dargestellten Ergebnisse einen vermehrt komplikationsbehafteten postoperativen Aufenthalt der Patienten mit fortgeschrittener Appendizitis, verglichen mit Patienten ohne Perforation und ohne Peritonitis. Dies schlägt sich in einer durchschnittlich 5 Tage längeren postoperativen Verweildauer und einem Anstieg der Komplikationsrate auf etwa $20 \%$ bei Patienten mit schweren Appendizitisformen nieder. Übereinstimmend mit diesen Ergebnissen berichten Eldar et al. von einer deutlich erhöhten infektiösen Komplikationsrate von $20 \%$ und einer durchschnittlich 3 Tage längeren stationären Verweildauer der Patienten mit perforierter Appendizitis (Eldar et al. 1997). Der Zusammenhang von verlängertem postoperativem Intervall oder vermehrt infektiösen Komplikationen bei fortgeschrittenem Appendizitisbefund wird auch in weiteren Untersuchungen bestätigt (Ditillo et al. 2006; Teixeira et al. 2012). Insbesondere stiegen im untersuchten Patientenkollektiv die Rate an Wundheilungsstörungen, Revisionsoperationen und sonstigen Komplikationen an. Des Weiteren war aufgrund des fortgeschrittenen intraoperativen Befundes häufiger eine offene Appendektomie und auch eine deutlich längere Operationsdauer erforderlich. Diese Ergebnisse stehen im Einklang mit den Erkenntnissen von Bhangu und Teixeira et al. (Teixeira et al. 2012; Bhangu 2014).

Es lässt sich nach Zusammenschau der Ergebnisse dieser Untersuchung und der Literaturrecherche schlussfolgern, dass die Perforationsrate durch eine verkürzte Aufnahme-SchnittDauer nicht deutlich gesenkt werden kann. Die zeitliche Entwicklung von schweren Appendizitisformen scheint vermehrt von der Länge der Symptomdauer bis zur Patientenvorstellung als von klinikumsinternen präoperativen Verzögerungen beeinflusst zu sein. Obwohl somit der Einfluss des Chirurgen auf die Perforationsrate gering ist, bleibt die zügige Indikationsstellung im klinischen Alltag entscheidend, um das Auftreten vermeidbarer - erst nach Patientenaufnahme entstandener - Appendixperforationen zu verhindern. Dies ist insbesondere vor dem Hinter- 
grund der erhöhten Komplikationsrate bei fortgeschrittener Appendizitis von großer klinischer Relevanz.

\section{3 Übereinstimmung zwischen chirurgischem und histologischem Befund}

Bei Patienten ohne klinisch eindeutige Zeichen einer akuten Appendizitis liegt der Entschluss zur operativen Intervention nahe, wenn die Möglichkeit erwogen wird einen fortgeschrittenen Entzündungsbefund zu übersehen. Eine diagnostische Laparoskopie bietet den Vorteil einer direkten Inspektion der Appendix und der umliegenden Bauchorgane, sodass neben der Verdachtsdiagnose der Appendizitis auch eventuelle Differentialdiagnosen validiert werden können. Intraoperativ entscheidet der Chirurg zwischen Entfernen oder Belassen der Appendix, wobei die heute häufige Durchführung von laparoskopischen Appendektomien in Deutschland (Sahm et al. 2013) diese Entscheidungsfreiheit ermöglicht. Während im Zeitalter der ausschließlich offen durchgeführten Appendektomien die Schnittsetzung gleichbedeutend mit einer Appendektomie war - um späterer differentialdiagnostische Verwirrung durch Vorhandensein der Appendix trotz typischer Unterbauchlaparotomienarbe vorzubeugen - lässt der laparoskopische Zugang dank minimaler und weniger charakteristischer Narbenbildung auch ein rein inspizierendes Vorgehen zu (Garlipp und Arlt 2009; Wente und Waleczek 2009). Wie Slotboom et al. zeigen konnten, beruht dabei die Entscheidung des Chirurgen für oder gegen eine Appendektomie in erster Linie auf der Erkennbarkeit von visuell wahrnehmbaren Entzündungszeichen (Slotboom et al. 2014). Negative Appendektomieraten von bis zu 25 \% (Zielke 2002) unterstreichen die Notwendigkeit einer sicheren makroskopischen Beurteilung der Appendix, suggerieren jedoch auch Appendektomien reizloser Appendizes im Rahmen durchgeführter Laparoskopien bei Verdacht auf Appendizitis. Komplikationsraten, stationäre Aufenthaltsdauer des Patienten und Revisionsoperationsraten sind nach Bijnen et al. leicht erniedrigt bei rein diagnostischer Laparoskopie im Vergleich zur „unnötigen“ Entfernung einer reizlosen Appendix (Bijnen et al. 2003), wobei die klinische Relevanz dieser minimalen Unterschiede von Garlipp und Arlt in Frage gestellt wird (Garlipp und Arlt 2009). Bei irrtümlicher Belassung einer entzündeten Appendix ist hingegen von der Notwendigkeit zur Revisionsoperation mit erhöhter Komplikationsgefahr und Mehrkostenbelastung auszugehen. Diese Gründe sprechen für die dringliche Untersuchung der Korrektheit der intraoperativen Diagnosestellung.

Die Ergebnisse des Vergleichs zwischen intraoperativem und histologischem Befund lassen auf ein hohes Maß an Übereinstimmung zwischen chirurgischer und pathologischer Appendixbeurteilung im untersuchten Patientenkollektiv schließen (79,8 \%). In anderen Studien wurden Werte von 80 \% bis 88 \% (Hussain et al. 2009; Strong et al. 2015) veröffentlicht, welche mit die- 
sen Ergebnissen vergleichbar sind. Differierten intraoperativer und histologischer Befund, so diagnostizierte der Chirurg überwiegend eine Entzündung der Appendix, die im Nachhinein histologisch nicht bestätigt wurde (17,9\%). In anderen Publikationen werden hierbei niedrigere Werte von 8 \% (Strong et al. 2015) bis $11 \%$ (Shum et al. 2005) genannt. Dabei ist zu beachten, dass entsprechend der Beschreibung in Kapitel 4.4 im untersuchten Patientenkollektiv die histologische Diagnose einer „reizlosen“ Appendix neben blanden auch chronisch entzündete und vernarbte Appendixzustände umfasste, die möglicherweise der makroskopischen Beurteilung durch den Chirurgen nicht entgingen, jedoch als Manifestation einer akuten Entzündung gedeutet wurden. Des Weiteren ist zu erwähnen, dass die histologische Diagnose einer neurogenen Appendikopathie nicht in die Analyse der Patientendaten aufgenommen wurde, da sie lediglich bei 5 Patienten des Gesamtkollektivs im Zusammenhang mit einer deutlichen Fibrosierung des Appendixlumens genannt wurde. Mit 0,8 \% liegt somit der Anteil von Patienten mit dieser Erkrankung im untersuchten Patientenkollektiv deutlich unter der von Güller et al. beschriebenen Häufigkeit von 17,1 \% (Güller et al. 2001) und erscheint damit zu gering, um statistisch gesicherte Aussagen zum Vorkommen der neurogenen Appendikopathie im Gesamtkollektiv abzuleiten. Anhand dieses ausgesprochen geringen prozentualen Anteils kann spekuliert werden, ob die Erkrankung im Rahmen der histologischen Routineuntersuchung in der durchgeführten Studie möglicherweise größtenteils nicht erfasst wurde. Dies könnte einerseits mit den unterschiedlichen Färbemethoden zu begründen sein, die in Publikationen zur Diagnosestellung der neurogenen Appendikopathie untersucht wurden (Franke et al. 2001; Partecke et al. 2013). Andererseits existieren diverse Publikationen mit teils widersprüchlichen Theorien zur Pathogenese dieser Erkrankung (Di Sebastiano et al. 1999; Partecke et al. 2013), die das Auffinden geeigneter eindeutiger histologischer Parameter erschweren. Die Diagnose einer neurogenen Appendikopathie kann ausschließlich histologisch erfolgen (Güller et al. 2001), während die klinischen Symptome denen der akuten Appendizitis gleichen (Franke et al. 2001; Partecke et al. 2013). Der hohe Anteil an operativ entfernten, aber letztlich als histologisch reizlos eingestuften Appendizes in dieser Untersuchung könnte demnach auch mit einer fehlenden Erfassung neurogener Appendikopathien verknüpft sein. Der umgekehrte Fall einer intraoperativen Einschätzung der Appendix als blande bei nachfolgend histologisch manifesten akuten Entzündungszeichen trat nur selten auf (2,3\%). In anderen Studien wird ein höherer Anteil an intraoperativ fälschlicherweise als reizlos bezeichneten Appendizes angegeben. Hier reichen die Angaben von 2,7\% (Shum et al. 2005), über 4,4 \% (Strong et al. 2015) bis hin zu $9 \%$ (Hussain et al. 2009).

Die sich aus diesen Ergebnissen ergebenden Konsequenzen werden in der Literatur ausgesprochen kontrovers diskutiert. Einige Autoren fordern eine routinemäßige Appendektomie unabhängig von der makroskopischen Appendixbeurteilung, um auszuschließen, dass eine nur mikroskopisch erkennbare Entzündung unbemerkt bleibt (Roberts et al. 2008; Garlipp und Arlt 2009; Hussain et al. 2009; Strong et al. 2015). Die klinische Relevanz solcher in der Regel mini- 
mal entzündlichen Veränderungen ist jedoch nicht sicher (Garlipp und Arlt 2009) und die daraus abgeleitete Forderung nach einem Belassen der intraoperativ reizlos erscheinenden Appendix in anderen Publikationen (van den Broek et al. 2001) verdeutlicht die kontroverse Diskussion dieser Thematik. Die Frage, ob eine routinemäßige Appendektomie gerechtfertigt ist, kann aufgrund der ambivalenten Studienlage demnach nicht mit letzter Sicherheit geklärt werden. Jedoch sprechen eine im untersuchten Kollektiv verhältnismäßig geringe, aber messbare Restungenauigkeit intraoperativ nicht diagnostizierter Appendizitiden sowie makroskopisch nicht diagnostizierbare neurogene Appendikopathien eher für eine grundsätzliche Appendektomie, zumal keine relevanten Nachteile von negativ appendektomierten Patienten belegt sind. Diese konnten auch in diesem Kollektiv nicht nachgewiesen werden. Patienten mit negativer Appendektomie wiesen keine erhöhte postoperative Komplikationsrate oder verlängerte stationäre Verweildauer im Vergleich zu Patienten mit akuter Appendizitis auf. Es kann spekuliert werden, ob die aufgetretenen Komplikationen weniger auf die Entfernung der reizlosen Appendix als vielmehr auf den operativen Eingriff an sich zurückzuführen sind (Garlipp und Arlt 2009). Diesbezüglich argumentieren Wente und Waleczek jedoch, dass auch verhältnismäßig niedrige postoperative Komplikationsraten bei überflüssiger Entfernung eines letztlich gesunden Organs trotzdem immer als zu hoch angesehen werden müssen (Wente und Waleczek 2009).

Ausgesprochen sicher war der Chirurg in dieser Untersuchung in der Diagnose einer Appendixperforation. Je fortgeschrittener das Appendizitisstadium war, desto besser kongruierten histologischer und intraoperativer Befund. Roberts et al. bestätigen diese Ergebnisse. Fortgeschrittene Appendizitisstadien wie Perforation, Abszessbildung und gangränöser Zerfall wurden in jener Studie mit deutlich größerer Treffsicherheit als leichte Entzündungsvorgänge oder sogar blande Appendizes diagnostiziert (Roberts et al. 2008).

Im untersuchten Patientenkollektiv ist eine Tendenz zur "großzügigen“ Diagnose einer Appendizitis und damit Indikation zur Appendektomie durch den Chirurgen erkennbar. Im Zweifel entschied dieser sich zugunsten einer Appendixentfernung. Slotboom et al. bestätigen diesen Grundsatz in einer niederländischen Studie, nach der $51 \%$ der histologisch negativ befundeten Appendizes intraoperativ sicher als entzündet beurteilt wurden. In $33 \%$ der Fälle war der Chirurg unsicher in seiner Entscheidung, entschloss sich dann aber sicherheitshalber zur Appendektomie (Slotboom et al. 2014). Eine weitere Studie räumt eine Tendenz des Chirurgen zur Überschätzung des Appendizitisschweregrades ein (Colleran et al. 2007). Die Erklärung hierfür könnte in der Art der Entscheidungsfindung des Chirurgen liegen. Da dieser den Patienten nicht zum ersten Mal im Operationssaal antrifft, bleibt eine Beeinflussung durch präoperativ erhobene Befunde und klinisches Erscheinungsbild des Patienten nicht aus. Die Entscheidung für eine Appendektomie wird vom Chirurgen demnach nicht isoliert anhand der intraoperativen makroskopischen Appendixdarstellung, sondern nach Zusammenschau aller zur Verfügung stehenden Parameter getroffen. Nach Güller et al. liegt der Anteil von neurogenen Appendikopathien bei 
reizlos erscheinenden Appendizes trotz Appendizitissymptomatik bei 53 \% (Güller et al. 2001). Dies rechtfertigt die Notwendigkeit einer intraoperativen Entscheidung zur Appendektomie nicht nur in Abhängigkeit vom makroskopischen Erscheinungsbild der Appendix vermiformis, sondern auch in Abhängigkeit von der jeweiligen präoperativen Befundkonstellation. Des Weiteren kann eine fehlende Bestätigung von überprüften Differentialdiagnosen zum chirurgischen Entschluss für eine Appendektomie führen. Auch ist zu vermuten, dass der Chirurg eher auf die Diagnose einer akuten Appendizitis abzielt - aus der sich konsequenterweise auch die Indikation zur Appendektomie ergibt - um seine initiale Entscheidung zur Operation zu rechtfertigen.

Zusammenfassend lässt sich ein hohes Maß an Übereinstimmung zwischen chirurgischer und histologischer Einschätzung der Appendizitis postulieren. Die Richtigkeit der chirurgischen Diagnose war dabei abhängig vom Schweregrad der Entzündung. Eine chirurgische Überschätzung des Entzündungsschweregrades trat häufiger als eine intraoperativ nicht erfasste Appendizitis auf. Diese geringe aber dennoch vorhandene Wahrscheinlichkeit einen Entzündungszustand der Appendix vermiformis intraoperativ zu übersehen beziehungsweise die Möglichkeit eine Appendix mit makroskopisch nicht diagnostizierbarer neurogener Appendikopathie in situ zu belassen, legt die Forderung nach routinemäßiger Appendektomie unabhängig vom makroskopischen Erscheinungsbild der Appendix nahe, wobei hierzu aufgrund der ambivalenten Studienlage keine eindeutige Empfehlung ausgesprochen werden kann und weitere klinische Untersuchungen abgewartet werden müssen.

\subsection{Einschränkungen der Methodik}

Vor dem Hintergrund der retrospektiven Datenerhebung in Form einer Single-Center-Studie müssen einige Einschränkungen der Aussagekraft der dieser Studie zugrunde liegenden Daten erörtert werden.

Aufgrund der ausschließlichen Berücksichtigung von an der Universitätsmedizin Göttingen therapierten Patienten ist von einer eingeschränkten Übertragbarkeit der Ergebnisse auf andere Institutionen auszugehen. Auch wurde der postoperative Patientenaufenthalt des Gesamtkollektivs lediglich innerhalb dieses Krankenhauses nachverfolgt, sodass sich eventuelle Komplikationen, die Patienten nicht zur Wiedervorstellung an der Universitätsmedizin Göttingen veranlassten, nicht im untersuchten Patientenkollektiv niederschlugen. Das gewählte retrospektive Studiendesign besitzt den Vorteil einer Datenerhebung aus einem umfangreichen und abgeschlossen therapierten Patientenkollektiv, wodurch eine vollständige Nachvollziehbarkeit des stationären Krankheitsverlaufs ermöglicht wird. Nachteilig wirkt sich die fehlende Standardisierung der so erhobenen Daten aus, die sich in diesem Kollektiv in partieller Unvollständigkeit oder behandlerabhängiger Uneinheitlichkeit der Befunde manifestierte und eine Interpretation von 
deskriptiven Berichten sowie Berechnungen von nicht genannten Werten notwendig machte. So war mitunter beispielsweise nicht erkennbar, ob ein nicht genannter Parameter innerhalb eines Befundes für negativ befunden oder schlichtweg nicht untersucht worden war. Auch mussten subjektive Patientenangaben wie Symptomdauer oder Schmerzqualität auf Grundlage umfangreicher Beschreibungen im Aufnahme-Befund interpretiert werden, um Vergleichbarkeit herzustellen. Um eine Kongruenz von histologischem und intraoperativem Befund zu schaffen, erfolgte die Vereinheitlichung der verwendeten heterogenen Nomenklatur. So musste die histologische Beschreibung einer katarrhalischen Appendizitis mit dem intraoperativen Befund einer fibrinösen Appendizitis verglichen werden. Außerdem umfasste die unter dem histologischen Terminus „reizlos“ eingeordnete Appendix sowohl blande als auch chronisch entzündete und vernarbte Appendixzustände, um eine Vergleichbarkeit zur intraoperativen Diagnose der blanden Appendix, die keine derartige Differenzierung aufwies, zu schaffen. Retrospektiv erhobene, nicht standardisierte und damit unvollkommene Daten spiegeln andererseits die tatsächlichen Abläufe innerhalb eines Krankenhauses wider, sodass anhand der vorliegenden Daten realistische qualitative Aussagen zur Appendizitistherapie an der Universitätsmedizin Göttingen getroffen werden können. So beeinflusste die Wahl der Krankenhausabteilung, welche mit der initialen Versorgung nach Patientenvorstellung betraut wurde, die Geschwindigkeit der Indikationsstellung zur Operation. Dies erklärt die erhebliche Streubreite der Aufnahme-Schnitt-Dauer des untersuchten Patientenkollektivs. Das Gesamtkollektiv dieser Studie bestand ungeachtet der Tatsache, dass die akute Appendizitis eine Erkrankung des jungen Menschen ist, aus Kindern und Erwachsenen, da alle Patienten im Untersuchungszeitraum in die Datenerhebung einbezogen wurden. Diese Kollektivzusammensetzung muss in der Datenauswertung ebenfalls beachtet werden, da die akute Appendizitis bei jungen und älteren Menschen variable Manifestationen und Verläufe aufweisen kann. Auch müssen mitunter andersartige Patientengefüge der in den Kapiteln 4.1, 4.2 und 4.3 zitierten Quellen im Vergleich zum untersuchten Gesamtkollektiv berücksichtigt werden. 


\section{Zusammenfassung}

Die akute Appendizitis ist eine der häufigsten Ursachen für ein akutes Abdomen. Ihre sichere Diagnose und Therapie obliegt der chirurgischen Verantwortung und besitzt einen hohen Stellenwert im Rahmen der chirurgischen Notfallversorgung. Aufgrund der vielgestaltigen klinischen Manifestation der akuten Appendizitis besteht die Notwendigkeit zuverlässige präoperative Prädiktoren und einen klar definierten Zeitpunkt zur chirurgischen Therapie zu ermitteln sowie die Zuverlässigkeit der intraoperativen Appendixbeurteilung zu untersuchen.

Im Rahmen einer retrospektiven Datenerhebung wurden alle im Zeitraum von Januar 2008 bis April 2013 an der Universitätsmedizin Göttingen appendektomierten Patienten in die Untersuchung einbezogen. Ausgeschlossen wurden Patienten mit Gelegenheitsappendektomien und Operationen ohne Verdachtsdiagnose einer akuten Appendizitis. Es erfolgte die Erhebung definierter demographischer, anamnestischer, klinischer, diagnostischer, peri- und postoperativer Daten sowie die Kollektivuntersuchung durch Subgruppenanalysen und Vergleiche von intraoperativen und histologischen Befunden.

Das Gesamtkollektiv umfasste 655 Patienten. Das Durchschnittsalter betrug 28,4 $\pm 19,2$ Jahre, wobei weibliche Patienten mit $52 \%$ etwas stärker vertreten waren. Intraoperativ wurde bei 581 und histologisch bei 487 Patienten eine akute Appendizitis diagnostiziert. Postoperative Komplikationen traten in 60 Fällen auf. Eine intraoperative Appendixperforation wiesen 103 Patienten und eine Peritonitis 102 Personen auf. Die durchschnittliche Aufnahme-Schnitt-Dauer betrug 14,7 \pm 22 ,2 Stunden. Anhand der Datenanalyse ist erkennbar, dass akute Appendizitiden und postoperativ komplikationsbehaftete Krankheitsverläufe durch anamnestische Patientenbefragung, Parameter der klinischen Untersuchung, der Abdomensonographie und der Laborchemie prognostiziert werden können, wobei weniger ein einzelner sicherer Prädiktor als die Zusammenschau der Befundkonstellation entscheidend für die korrekte Diagnose ist. Die Studie legt weiterhin nahe, dass die Diagnose einer akuten Appendizitis eine Indikation zur zeitnahen Operation darstellt, da fortgeschrittene Appendizitiden mit erhöhten postoperativen Komplikationsraten vergesellschaftet sind. Die Perforationsrate kann durch einen zügigen Operationsbeginn allerdings nicht deutlich gesenkt werden. Das in dieser Untersuchung beschriebene vorhandene Risiko eine intraoperativ makroskopisch nicht erkennbar entzündete Appendix zu belassen, legt die Forderung einer routinemäßigen Appendektomieindikation nahe, wobei die ausgesprochen ambivalente Studienlage zu dieser Frage keine eindeutige Empfehlung zulässt.

Die akute Appendizitis ist eine häufig auftretende Erkrankung, deren Diagnose ein hohes Maß an klinischer Erfahrung auf Seiten des Behandlers verlangt und welche eine Indikation zur zeitnahen Operation darstellt. 


\section{Literaturverzeichnis}

Abou-Nukta F, Bakhos C, Arroyo K, Koo Y, Martin J, Reinhold R, Ciardiello K (2006): Effects of delaying appendectomy for acute appendicitis for 12 to 24 hours. Arch Surg $1960 \underline{141}$ 504-506

Addiss D, Shaffer N, Fowler B, Tauxe R (1990): The epidemiology of appendicitis and appendectomy in the United States. Am J Epidemiol $132,910-925$

Al-Abed Y, Alobaid N, Myint F (2014): Diagnostic markers in acute appendicitis. Am J Surg 29, 15

Al-Omran M, Mamdani M, McLeod R (2003): Epidemiologic features of acute appendicitis in Ontario, Canada. Can J Surg 46, 263-268

Anderson J, Bickler S, Chang D, Talamini M (2012): Examining a common disease with unknown etiology: trends in epidemiology and surgical management of appendicitis in California, 1995-2009. World J Surg 36, 2787-2794

Andersson R (2004): Meta-analysis of the clinical and laboratory diagnosis of appendicitis. $\mathrm{Br} \mathrm{J}$ Surg $91,28-37$

Andersson R, Hugander A, Ghazi S, Ravn H, Offenbartl S, Nyström P, Olaison G (1999): Diagnostic value of disease history, clinical presentation and inflammatory parameters of appendicitis. World J Surg 23, 133-140

Andreu-Ballester J, González-Sánchez A, Ballester F, Almela-Quilis A, Cano-Cano M, MillanScheiding M, Ruiz del Castillo J (2009): Epidemiology of appendectomy and appendicitis in the Valencian community (Spain), 1998-2007. Dig Surg 26, 406-412

Bates M, Khander A, Steigman S, Tracy T, Luks F (2014): Use of white blood cell count and negative appendectomy rate. Pediatrics 133 e39-e44

Becker K, Höfler H (2002): Pathologie der Appendizitis. Chirurg 73, 777-781

Bhangu A (2014): Safety of short, in-hospital delays before surgery for acute appendicitis: multicentre cohort study, systematic review and meta-analysis. Ann Surg 259, 894-903

Bijnen C, van den Broek W, Bijnen A, de Ruiter P, Gouma D (2003): Implications of removing a normal appendix. Dig Surg 20, 215-219

Binnebösel M, Otto J, Stumpf M, Mahnken A, Gaßler N, Schumpelick V, Truong S (2009): Akute Appendizitis. Moderne Diagnostik - der chirurgische Ultraschall. Chirurg $\underline{80}$ 579-587 
Birnbaum B, Wilson S (2000): Appendicitis at the millennium. Radiology 215, 337-348

Boenigk H, Meyer F, Koch A, Gastinger I (2012): Surgical determinants, perioperative course and outcome of a representative patient cohort with acute appendicitis undergoing appendectomy over 3 decades. Pol Przegl Chir $\underline{84}$ 509-520

Chung J, Kong M, Lin S, Lin T, Huang C, Lou C, Lin J (1996): Diagnostic value of C-reactive protein in children with perforated appendicitis. Eur J Pediatr 155, 529-531

Clyde C, Bax T, Merg A, MacFarlane M, Lin P, Beyersdorf S, McNevin M (2008): Timing of intervention does not affect outcome in acute appendicitis in a large community practice. $A m J$ Surg 195, 590-592

Colleran G, Heneghan H, Sweeney K, Kerin M (2007): A comparison of surgical impression, histological findings and microbiological results at open appendicectomy. Ir Med J 100 593596

Di Sebastiano P, Fink T, di Mola F, Weihe E, Innocenti P, Friess H, Büchler M (1999): Neuroimmune appendicitis. Lancet $\underline{354}$ 461-466

Ditillo M, Dziura J, Rabinovici R (2006): Is it safe to delay appendectomy in adults with acute appendicitis? Ann Surg 244, 656-660

Dixon J, Elton R, Rainey J, Macleod D (1991): Rectal examination in patients with pain in the right lower quadrant of the abdomen. BMJ $\underline{302}$,386-388

Doria A, Moineddin R, Kellenberger C, Epelman M, Beyene J, Schuh S, Babyn P, Dick P (2006): US or CT for diagnosis of appendicitis in children and adults? A meta-analysis. Radiology 241 83-94

Drake F, Mottey N, Farrokhi E, Florence M, Johnson M, Mock C, Steele S, Thirlby R, Flum D (2014): Time to appendectomy and risk of perforation in acute appendicitis. JAMA $\underline{149}$ 837-844

Eastridge B, Hamilton E, O'Keefe G, Rege R, Valentine R, Jones D, Tesfay S, Thal E (2003): Effect of sleep deprivation on the performance of simulated laparoscopic surgical skill. Am J Surg 186, $169-174$

Eldar S, Nash E, Sabo E, Matter I, Kunin J, Mogilner J, Abrahamson J (1997): Delay of surgery in acute appendicitis. Am J Surg $\underline{173}, 194-198$

Faiz O, Clark J, Brown T, Bottle A, Antoniou A, Farrands P, Darzi A, Aylin P (2008): Traditional and laparoscopic appendectomy in adults. Outcomes in English NHS hospitals between 1996 and 2006. Ann Surg 248, 800-806 
Franke C, Böhner H, Yang Q, Ohmann C, Röher H (1999): Ultrasonography for diagnosis of acute appendicitis: results of a prospective multicenter trial. World J Surg 23 , 141-146

Franke C, Gerharz C, Böhner H, Ohmann C, Heydrich G, Krämling H, Stock W, Rosen D, Kurpreugsch K, Röher H (2001): Neurogenic appendicopathy: a clinical disease entity? Int J Colorectal Dis 17 , 185-191

Galindo Gallego M, Fadrique B, Nieto M, Calleja S, Fernández-Aceñero M, Ais G, González J, Manzanares J (1998): Evaluation of ultrasonography and clinical diagnostic scoring in suspected appendicitis. BrJ Surg $\underline{85}, 37-40$

Garlipp B, Arlt G (2009): Laparoskopie bei Verdacht auf akute Appendizitis. Soll die makroskopisch unauffällige Appendix entfernt werden? Chirurg $\underline{80}$, 615-621

Gauderer M, Crane M, Green J, DeCou J, Abrams R (2001): Acute appendicitis in children: the importance of family history. J Pediatr Surg $\underline{36}, 1214-1217$

Grönroos J, Grönroos P (1999): Leucocyte count and C-reactive protein in the diagnosis of acute appendicitis. BrJ Surg $\underline{86}, 501-504$

Güller U, Oertli D, Terracciano L, Harder F (2001): Neurogene Appendikopathie: Ein häufiges, fast unbekanntes Krankheitsbild. Auswertung von 816 Appendizes und Literaturübersicht. Chir $\underline{72}, 684-689$

Guller U, Hervey S, Purves H, Muhlbaier L, Peterson E, Eubanks S, Pietrobon R (2004): Laparoscopic versus open appendectomy. Outcomes comparison based on a large administrative database. Ann Surg 239, 43-52

Gupta A, Regmi S, Hazra N, Panhani M, Talwar O (2010): Clinically monitored delay - A valid option in cases with doubtful diagnosis of acute appendicitis. Indian J Surg $\underline{72}, 215-219$

Hamill J, Liley A, Hill A (2014): Historical aspects of appendicitis in children. ANZ J Surg $\underline{84}$, 307310

Harrison S, Benziger H: Diagnostic challenges in acute appendicitis. In: Lander A (Hrsg.): Appendicitis - A collection of essays from around the world. InTech-Verlag, Rijeka 2012, 21-42

Hollerweger A (2006): Sonographie der akuten Appendizitis. Ultraschall Med 27, 412-432

Hornby S, Shahtahmassebi G, Lynch S, Ladwa N, Stell D (2014): Delay to surgery does not influence the pathological outcome of acute appendicitis. Scand J Surg 103, 5-11

Horstmann R, Tiwisina C, Classen C, Palmes D, Gillessen A (2005): Laparoskopische vs. offene Appendektomie: Welche Faktoren beeinflussen die Wahl der Operationsmethode? Zentralbl Chir $130,48-54$ 
Humes D, Simpson J (2006): Acute appendicitis. BMJ $\underline{333}$, 530-534

Hussain A, Mahmood H, Singhal T, Balakrishnan S, El-Hasani S (2009): What is positive appendicitis? A new answer to an old question. Clinical, macroscopical and microscopical findings

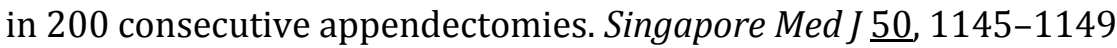

Isenmann R, Dürig M, Gebhardt H: Appendix. In: Henne-Bruns D, Kremer B, Dürig M (Hrsg.): Duale Reihe Chirurgie. Georg Thieme Verlag, Stuttgart 2008, 368-377

Junginger T, Küchle R (1998): Der Einfluss des Operateurs auf die Behandlungsqualität bei akuter Appendizitis. Chirurg 69, 432-437

Kessler N, Cyteval C, Gallix B, Lesnik A, Blayac P, Pujol J, Bruel J, Taourel P (2004): Appendicitis: evaluation of sensitivity, specificity, and predictive values of US, Doppler US and laboratory findings. Radiology $\underline{230}, 472-478$

Khanal B, Ansari M, Pradhan S (2008): Accuracy of ultrasonography in the diagnosis of acute appendicitis. Kathmandu Univ Med J 6 , 70-74

Koch A, Zippel R, Marusch F, Schmidt U, Gastinger I, Lippert H (2000): Prospective multicenter study of antibiotic prophylaxis in operative treatment of appendicitis. Dig Surg 17 370378

Körner H, Söreide J, Pedersen E, Bru T, Söndenaa K, Vatten L (2001): Stability in incidence of acute appendicitis. A population-based longitudinal study. Dig Surg 18, 61-66

Kreis M, von Koch F, Jauch K, Friese K (2007): Abklärung des rechtsseitigen Unterbauchschmerzes. Dtsch Ärztebl 104, 42a-42g

Langner H, Gabbert H: Appendix. In: Böcker W, Denk H, Heitz P, Höfler G, Kreipe H, Moch H (Hrsg.): Pathologie. Urban und Fischer, München 2012, 589-593

Laurell H, Hansson L, Gunnarsson U (2013): Manifestations of acute appendicitis: a prospective study on acute abdominal pain. Dig Surg $\underline{30}$ 198-206

Lee S, Walsh A, Ho H (2001): Computed tomography and ultrasonography do not improve and may delay the diagnosis and treatment of acute appendicitis. Arch Surg $\underline{136}, 556-562$

Lippert H, Koch A, Marusch F, Wolff S, Gastinger I (2002): Offene vs. laparoskopische Appendektomie. Chirurg 73, 791-798

Livingston E, Woodward W, Sarosi G, Haley R (2007): Disconnect between incidence of nonperforated and perforated appendicitis. Implications for pathophysiology and management. Ann Surg 245, 886-892 
Magdeburg R, Kähler G (2013): Neue Wege in der Behandlung der akuten Appendizitis? Zentralbl Chir 138, 284-288

Manner M, Stickel W (2001): Diagnostik bei Verdacht auf Appendizitis - Lässt sich eine akute Appendizitis sonographisch ausschließen? Chirurg $\underline{72}$, 1036-1042

Mirow L, Schiedeck T: Kolon, Appendix, Rektum und Anus. In: Bruch H, Trentz O (Hrsg.): Berchtold Chirurgie. Urban und Fischer, München 2008, 833-869

Obinwa O, Peirce C, Cassidy M, Fahey T, Flynn J (2015): A model predicting perforation and complications in paediatric appendicectomy. Int J Colorectal Dis $\underline{30}, 559-565$

Ohmann C, Franke C, Kraemer M, Yang Q (2002): Neues zur Epidemiologie der akuten Appendizitis. Chirurg $\underline{73}, 769-776$

Partecke L, Thiele A, Schmidt-Wankel F, Kessler W, Wodny M, Dombrowski F, Heidecke C, von Bernstorff W (2013): Appendicopathy - a clinical and diagnostic dilemma. Int J Colorectal Dis 28, 1081-1089

Peiper C: Appendizitis. In: Schumpelick V, Siewert J, Rothmund M (Hrsg.): Praxis der Viszeralchirurgie, Band 2: Gastroenterologische Chirurgie. Springer-Verlag, Heidelberg 2006, 487-495

Petroianu A (2012): Diagnosis of acute appendicitis. Int J Surg 10 115-119

Piper H, Rusnak C, Orrom W, Hayashi A, Cunningham J (2008): Current management of appendicitis at a community center - how can we improve? Am J Surg 195, 585-589

Reich B, Zalut T, Weiner S (2011): An international evaluation of ultrasound vs. computed tomography in the diagnosis of appendicitis. Int J Emerg Med $\underline{4}, 68-74$

Reissfelder C, Mc Cafferty B, von Frankenberg M (2009): Offene Appendektomie. Wann wird sie noch gebraucht? Chirurg $\underline{80}, 602-607$

Rettenbacher T, Hollerweger A, Macheiner P, Rettenbacher L, Tomaselli F, Schneider B, Gritzmann N (2001): Outer diameter of the vermiform appendix as a sign of acute appendicitis: evaluation at US. Radiology $\underline{218}, 757-762$

Roberts J, Behravesh M, Dmitrewski J (2008): Macroscopic findings at appendicectomy are unreliable: implications for laparoscopy and malignant conditions of the appendix. Int J Surg Pathol 16, 386-390

Sack U, Biereder B, Elouahidi T, Bauer K, Keller T, Tröbs R (2006): Diagnostic value of blood inflammatory markers for detection of acute appendicitis in children. BMC $\underline{6}, 15-22$ 
Sadot E, Wasserberg N, Shapiro R, Keidar A, Oberman B, Sadetzki S (2013): Acute appendicitis in the twenty-first century: should we modify the management protocol? J Gastrointest Surg 17, $1462-1470$

Sahm M, Pross M, Lippert H (2011): Akute Appendizitis - Wandel in Epidemiologie, Diagnostik und Therapie. Zentralbl Chir 136, 18-24

Sahm M, Koch A, Schmidt U, Wolff S, Pross M, Gastinger I, Lippert H (2013): Akute Appendizitis Klinische Versorgungsforschung zur aktuellen chirurgischen Therapie. Zentralbl Chir 138 , 270-277

Semm K (1983): Endoscopic appendectomy. Endoscopy 15, 59-64

Shum C, Lim J, Soo K, Wong W (2005): On-table diagnostic accuracy and the clinical significance of routine exploration in open appendectomies. Asian J Surg 28, 257-261

Slotboom T, Hamminga J, Hofker H, Heineman E, Haveman J (2014): Intraoperative motive for performing a laparoscopic appendectomy on a postoperative histological proven normal appendix. Scand J Surg 103, 245-248

Stein G, Rath-Wolfson L, Zeidman A, Atar E, Marcus O, Joubran S, Ram E (2012): Sex differences in the epidemiology, seasonal variation and trends in the management of patients with acute appendicitis. Langenbecks Arch Surg $\underline{397}$ 1087-1092

Strong S, Blencowe N, Bhangu A (2015): How good are surgeons at identifying appendicitis? Results from a multi-centre cohort study. Int J Surg 107, 107-112

Sulu B, Günerhan Y, Palanci Y, Işler B, Cağlayan K (2010): Epidemiological and demographic features of appendicitis and influences of several environmental factors. Ulus Travma Acil Cer 16, 38-42

Teixeira P, Sivrikoz E, Inaba K, Talving P, Lam L, Demetriades D (2012): Appendectomy timing: waiting until the next morning increases the risk of surgical site infections. Ann Surg $\underline{256}$, 538-543

Temple C, Huchcroft S, Temple W (1995): The natural history of appendicitis in adults. A prospective study. Ann Surg 221, 278-281

Treutner K, Schumpelick V (1997): Epidemiologie der Appendizitis. Chirurg 68, 1-5

Van den Broek W, Bijnen A, de Ruiter P, Gouma D (2001): A normal appendix found during diagnostic laparoscopy should not be removed. BrJ Surg $\underline{88}, 251-254$

Van Randen A, Bipat S, Zwinderman A, Ubbink D, Stoker J, Boermeester M (2008): Acute appendicitis: meta-analysis of diagnostic performance of CT and graded compression US related to prevalence of disease. Radiology 249, 97-106 
Wagner M, Aronsky D, Tschudi J, Metzger A, Klaiber C (1996): Laparoscopic stapler appendectomy. A prospective study of 267 consecutive cases. Surg Endosc 10, 895-899

Wente M, Waleczek H (2009): Strategien zur Vermeidung negativer Appendektomien. Chirurg 80, 588-593

Zielke A (2002): Appendizitis. Moderne Diagnostik. Chirurg 73, 782-790

Zielke A, Sitter H, Rampp T, Bohrer T, Rothmund M (2001): Clinical decision-making, ultrasonography and scores for evaluation of suspected acute appendicitis. World J Surg $\underline{25}$, 578584

Zoarets I, Poluksht N, Halevy A (2014): Does selective use of computed tomography scan reduce the rate of „white“ (negative) appendectomy? Isr Med Assoc J 16, 335-337 


\section{Danksagung}

Ich möchte mich herzlich bei Herrn Prof. Dr. med. Kollmar für die Betreuung der Doktorarbeit und die wertvollen Anregungen und Vorschläge bei der Verfassung der Dissertation bedanken. Weiterhin danke ich Herrn Dr. med. Slotta für die Unterstützung bei der statistischen Auswertung der Daten. Aufs Herzlichste möchte ich mich darüber hinaus für seine großartige Betreuung, Ermutigung und konstruktive Kritik bedanken, mit der er mir stets eine große Hilfe war. Er stand mir bei Fragen jederzeit zur Verfügung und gewährte mir spannende Einblicke in ein Themengebiet außerhalb meines Fachbereichs, die ich nicht mehr missen möchte. 


\section{Lebenslauf}

Am 07.10.1989 wurde ich, Ulrike Kopsch, als erstes Kind meiner Eltern, die zum damaligen Zeitpunkt Medizin studierten, in Leipzig geboren.

Meine Grundschulzeit verbrachte ich von 1995 bis 2000 an der Grundschule Hegelstraße Magdeburg und wechselte danach auf das Ökumenische Domgymnasium Magdeburg. Mit dem Abitur schloss ich 2008 meine schulische Ausbildung ab und verbrachte bis 2009 ein berufliches Orientierungsjahr, in welchem ich diverse Praktika im medizinischen und zahnmedizinischen Bereich absolvierte. Des Weiteren führte ich einen viermonatigen Au-Pair Aufenthalt in London, England, durch.

Im Wintersemester 2009/2010 begann ich mein Studium der Zahnmedizin an der GeorgAugust-Universität Göttingen, welches ich am 20. November 2014 mit der Gesamtnote „sehr gut“ abschloss. Im Rahmen dieser Ausbildung konnte ich mit einer Auslandsfamulatur in Siem Reap, Kambodscha, im März und April 2014 einen Schwerpunkt in meiner zahnärztlichen Tätigkeit setzen. Ab Juni 2015 trat ich eine Anstellung als Assistenzzahnärztin in einer Allgemeinzahnärztlichen Praxis in Hanau an.

Meine musikalische Ausbildung im Klavierspiel und Gesang erhielt ich von 1994 bis 2008 am Georg-Phillip-Telemann-Konservatorium Magdeburg und auch heute noch gilt mein großes persönliches Interesse der Musik. 